\title{
A review on TVD schemes and a refined flux-limiter for
}

\author{
steady-state calculations
}

\author{
Di Zhang ${ }^{1, *}$, Chunbo Jiang ${ }^{1, *}$, Dongfang Liang ${ }^{2}$, Liang Cheng ${ }^{3,4}$ \\ ${ }^{1}$ State Key Laboratory of Hydroscience and Engineering, Tsinghua University, \\ Beijing 100084, China \\ ${ }^{2}$ Department of Engineering, University of Cambridge, Trumpington Street, \\ Cambridge CB2 1PZ, UK \\ ${ }^{3}$ School of Civil, Environmental and Mining Engineering, The University of Western \\ Australia, 35 Stirling Highway, Crawley, WA 6009, Australia \\ ${ }^{4}$ State Key Laboratory of Coastal and Offshore Engineering, Dalian University of Technology, \\ Dalian, 116024, China
}

\begin{abstract}
This paper presents an extensive review of most of the existing TVD schemes found in literature that are based on the One-step Time-space-coupled Unsteady TVD criterion (OTU-TVD), the Multi-step Time-space-separated Unsteady TVD criterion (MTU-TVD) and the Semi-discrete Steady-state TVD criterion (SS-TVD). The design principles of these schemes are examined in detail. It is found that the selection of appropriate flux-limiters is a key design element in developing these schemes. Different flux-limiter forms (CFL-dependent or CFL-independent, and various limiting criteria) are shown to lead to different performances in accuracy and convergence. Furthermore, a refined SS-TVD flux-limiter, referred to henceforth as TCDF (Third-order Continuously Differentiable Function), is proposed for steadystate calculations based on the review. To evaluate the performance of the newly proposed scheme, many existing classical SS-TVD limiters are compared with the TCDF in eight two-dimensional test cases. The numerical results clearly show that the TCDF results in an improved overall performance.
\end{abstract}

Keywords: total variation diminishing; high resolution scheme; normalized variable diagram; convection boundedness criterion; convection discretization; flux limiter

\footnotetext{
* Corresponding authors: State Key Laboratory of Hydroscience and Engineering, Tsinghua University, The Hydraulic Building, Beijing 100084, China. Phone: +86 01062781820.

E-mail address: zhangdi10@ mails.tsinghua.edu.cn (D. Zhang), jcb@ mail.tsinghua.edu.cn (C. Jiang).
} 


\section{Introduction}

Numerical simulation of convection-dominated flow phenomena remains one of the most challenging problems in computational fluid dynamics (CFD) [1-3]. On one hand, it is well known that conventional low-order (LO) schemes, such as the first-order upwind (FOU), HYBRID, and POWER-LAW schemes, although being highly stable and unconditionally bounded, suffer from excessive numerical diffusion [4-6]. On the other hand, while traditional high-order (HO) schemes, such as the central differencing (CDS), second-order upwind (SOU), cubic-upwind interpolation (CUI) and quadratic-upwind interpolation (QUICK) schemes, improve the accuracy and introduce less numerical diffusion relative to the LO schemes, they generally generate unphysical oscillations when the solution contains shocks or steep gradients of the transported variable because of their unbounded nature [7-9].

Equipping the $\mathrm{HO}$ schemes with the boundedness property results in the so-called high-resolution schemes (HRS), which are able to provide good resolution in the vicinity of the steep gradient region without introducing spurious oscillations, while at the same time give at least second-order accuracy in smooth regions [10-12]. In the past decades, several different series of HRS have been developed [13-15], such as the flux-corrected transport (FCT) schemes, the total variation diminishing (TVD) schemes, the normalized variable diagram (NVD) schemes, and the essentially non-oscillatory (ENO) schemes.

The FCT schemes, firstly introduced by Boris and Book and later enhanced by Zalesak and many other researchers [16-18], belong to the two-step-method group. In two-step methods, a provisional update from a low-order scheme is computed in the first step, and then, a limited amount of higher-order anti-diffusive flux is added in the second step to produce accurate and monotonic results. The NVD schemes, based on non-linear characteristics in the normalized variable diagram, employ the convection boundedness criterion (CBC) so as to provide accuracy, stability, monotonicity and algorithmic simplicity [2, 6, 19-24]. The explicit CBC proposed by Leonard [25] and the implicit CBC by Gaskell and Lau [26] are widely considered as the sufficient and necessary condition of explicit and implicit convection schemes, respectively, in order to achieve the local boundedness. It should be noted that all the NVD schemes considered in this paper have been converted into the flux-limiter form to allow direct comparison, owing to the unique relationship between the NVD and TVD spaces [27-28]. 
The TVD schemes, originally developed by Harten [29], are a group of the most popular HRS schemes for solving hyperbolic conservation laws. Generally speaking, depending on some critical conditions (e.g. whether a steep gradient or a discontinuity exists), a TVD flux-limiter often switches from a high-order scheme to a low-order diffusive/compressive scheme (or vice versa) in order to circumvent the aforementioned numerical dilemma. It is generally recognized that the TVD schemes possess several attractive features, such as strictly preserving the monotonicity property (delivering well-resolved non-oscillatory discontinuities), computational simplicity and efficiency (using a fixed compact 5-point stencil in each spatial direction), and at least second-order accuracy in smooth regions [30-38].

Unfortunately, classical TVD schemes suffer from a well-known inherent drawback, namely the clipping of extrema, and therefore cannot recover the full order of accuracy [39-40]. To remedy this problem, more elaborate ENO schemes [41-43] have been proposed, which are not required to decrease the local extrema at every single time step. Numerical comparisons show that high-order ENO schemes preserve better high-frequency information and perform better than the traditional TVD schemes. However, as pointed out by Arora and Roe [44], the TVD schemes, when delicately conceived, are not in fact inferior to the simple kinds of ENO schemes and can be substantially more efficient in terms of computational costs. Further, taking into account the fact that ENO schemes use a larger finite-difference stencil than the traditional TVD schemes (viz. at least 7-point vs. compact 5-point for each spatial direction), it becomes extremely challenging and sometimes even impossible to apply the former on arbitrary unstructured meshes [4, 6, 45-47]. Due to the aforementioned attributes of the TVD schemes and the drawbacks of the ENO schemes, the TVD schemes are actually among the most widely used high-resolution discretization methods in practical applications [48-50].

In this article, a large number of TVD schemes in the literature are reviewed and their design principles are analyzed. These schemes are grouped into three broad categories: the OTU-TVD, the MTU-TVD and the SS-TVD. In addition, a new CFLindependent flux-limiter (TCDF) is proposed for steady-state calculations within the SS-TVD framework, whose accuracy and convergence are compared with many existing flux-limiters of the same kind. The results obtained in all the test cases clearly demonstrate the attractiveness of the newly-developed TCDF.

\section{One-Step Time-Space-Coupled Unsteady TVD Schemes}


This section deals with the development of accurate OTU-TVD schemes for the numerical simulation of unsteady linear advection equations. Similar discussions can be found in Refs. [10, 12, 30, 32-34, 44, 51-53]. To present OTU-TVD schemes, we consider the numerical solution of the hyperbolic conservation law:

$$
q_{t}+(a q)_{x}=0
$$

where $q=q(x, t)$ denotes the dependent variable and $a$ is the advection velocity. Without loss of generality, we assume that the velocity is a positive constant $(a>0)$. The opposite case $(a<0)$ can be treated by symmetry at each cell interface. When considering the numerical discretization of Eq. (1), we define $\Delta t$ as the time step and $\Delta x$ as the control cell size. In addition, owing to the stability requirement, the CFL number, $v=a \Delta t / \Delta x$, is limited to the range of $[0,1]$.

\subsection{One-Step Time-Space-Coupled Unsteady Linear Schemes}

We will follow the Lax-Wendroff approach to introduce the high-order one-step timespace-coupled linear schemes by correcting the successive modified equations. Contrary to conventional finite difference approximations based on the Taylor series expansion in space, the Lax-Wendroff type methods originate from the Taylor series expansion in time $[10,13,52]$.

$$
q\left(x_{i}, t+\Delta t\right)=q\left(x_{i}, \Delta t\right)+\Delta t \frac{\partial q}{\partial t}+\frac{1}{2}(\Delta t)^{2} \frac{\partial^{2} q}{\partial t^{2}}+\frac{1}{6}(\Delta t)^{3} \frac{\partial^{3} q}{\partial t^{3}}+\cdots+\frac{1}{n !}(\Delta t)^{n} \frac{\partial^{n} q}{\partial t^{n}}
$$

If retaining only the first three terms on the right-hand side of Eq. (2), substituting the time derivatives with space derivatives derived from the original advection equation, and using second-order centered difference approximations for the corresponding space derivatives, we can get the explicit second-order Lax-Wendroff scheme:

$$
\begin{aligned}
q_{i}^{n+1} & =q_{i}^{n}-\Delta t \cdot\left[a\left(q_{i}^{n}\right)_{x}\right]+\frac{1}{2} a(\Delta t)^{2} \cdot\left[a\left(q_{i}^{n}\right)_{x x}\right] \cdots \\
& =q_{i}^{n}-\frac{a \Delta t}{2 \Delta x} \cdot\left(q_{i+1}^{n}-q_{i-1}^{n}\right)+\frac{a^{2} \Delta t^{2}}{2(\Delta x)^{2}} \cdot\left(q_{i+1}^{n}-2 q_{i}^{n}+q_{i-1}^{n}\right) \cdots
\end{aligned}
$$

where the subscript $i$ indicates the $i^{\text {th }}$ grid point and the superscript $n$ denotes the $n^{\text {th }}$ time step. For convenience, we convert the second-order Lax-Wendroff scheme (Eq. (3)) into the general explicit flux-conservative form: 


$$
q_{i}^{n+1}=q_{i}^{n}-\frac{a \Delta t}{\Delta x} \cdot\left(q_{i+1 / 2}^{n}-q_{i-1 / 2}^{n}\right)=q_{i}^{n}-v \cdot\left(q_{i+1 / 2}^{n}-q_{i-1 / 2}^{n}\right)
$$

where $v$ is the CFL number $v=a \Delta t / \Delta x$, and the variable value at the cell face for the second-order Lax-Wendroff scheme $\left(q_{i+1 / 2}^{L W}\right)$ can be written as:

$$
q_{i+1 / 2}^{L W}=q_{i}^{n}+\frac{1}{2}\left(q_{i+1}^{n}-q_{i}^{n}\right)-\frac{1}{2} \frac{a \Delta t}{\Delta x}\left(q_{i+1}^{n}-q_{i}^{n}\right)=q_{i}^{n}+\frac{(1-v)}{2}\left(q_{i+1}^{n}-q_{i}^{n}\right)
$$

Furthermore, substituting Eq. (3) into Eq. (2) and after some algebraic manipulation, we obtain the modified equation for this scheme:

$$
q_{t}+(a q)_{x}=a \frac{(\Delta x)^{2}}{6}\left(v^{2}-1\right) q_{x x x}+O\left((\Delta x)^{3},(\Delta t)^{3}\right)
$$

By spatially discretizing the right-hand side of Eq. (6) and subtracting it from the second-order Lax-Wendroff scheme, one obtains the well-known explicit third-order upwind-biased scheme with a numerical flux [10, 32-34, 44, 51]:

$$
\begin{aligned}
& q_{i+1 / 2}^{n}=q_{i}^{n}+\Phi\left(r_{i+1 / 2}\right) \frac{(1-v)}{2}\left(q_{i+1}^{n}-q_{i}^{n}\right) \\
& \Phi^{3}\left(r_{i+1 / 2}\right)=1-\frac{1+v}{3}\left(1-r_{i+1 / 2}\right)
\end{aligned}
$$

where $r_{i+1 / 2}$ denotes the ratio of the upwind to central gradients in the data, which is essentially a local measure of smoothness. For linear problems on uniform grids, the gradient ratio $r_{i+1 / 2}$ is defined as $r_{i+1 / 2}=\left(q_{i}-q_{i-1}\right) /\left(q_{i+1}-q_{i}\right)$.

Similar to the deduction of the third-order scheme, by correcting the successive error terms of the modified equations, even higher-order (than third) one-step LaxWendroff type schemes can be expressed in the usual explicit flux-limited form (Eq. (7)), in which the flux-limiter function $\left(\Phi\left(r_{i+1 / 2}\right)\right)$ determines the order of the scheme. However, such one-step schemes, if higher than 3-order, use a larger stencil than the usual 5-point stencil, as pointed out by Daru and Tenaud [10]. Since we would like to limit our discussions to the compact 5-point stencil schemes, these higher order schemes are not presented in this study. Interested readers are referred to [10], where numerical schemes of this kind up to seventh order have been derived.

It is obvious that, for the first three terms on the right-hand side of Eq. (3), if the second-order centered difference approximations are replaced by second-order onesided approximations of the derivatives, one gets the second-order Beam-Warming scheme [13]. Further, by taking the arithmetic mean of the Beam-Warming scheme and the Lax-Wendroff scheme, one obtains the Fromm scheme [33]. 


$$
\begin{aligned}
& \Phi^{B W}\left(r_{i+1 / 2}\right)=r_{i+1 / 2} \\
& \Phi^{\text {Fromm }}\left(r_{i+1 / 2}\right)=\left(1+r_{i+1 / 2}\right) / 2
\end{aligned}
$$

It is worthwhile to emphasize that the explicit one-step high-order schemes obtained in this way always have the same order of accuracy in both time and space. However, in view of their unbounded nature, these schemes, when directly implemented for unsteady computations, will inevitably produce spurious oscillations in the vicinity of discontinuities. Therefore, in order to avoid the generation of such unphysical oscillations, a monotonicity criterion will be introduced in the context of one-step unsteady calculations in the next sub-section.

\subsection{One-Step Unsteady TVD Criterion}

The total variation diminishing (TVD) concept, originally developed by Harten [29], is based on a rigorous mathematical foundation and provides a unifying framework for the construction of non-linear monotonicity-preserving high-resolution schemes for the one-dimension (1D) linear advection equation.

In one space dimension, the total variation of the discrete solution at time step $n$, denoted by $T V\left(q^{n}\right)$, can be written as:

$$
T V\left(q^{n}\right)=\sum_{i}\left(q_{i+1}^{n}-q_{i}^{n}\right)
$$

In essence, the total variation is a measure of the oscillatory character of the solution. According to Harten [29, 30], the general explicit flux-conservative form (Eq. (4)) is said to be TVD if the total variation does not grow with time.

$$
T V\left(q^{n+1}\right) \leq T V\left(q^{n}\right)
$$

For the one-dimensional (1D) hyperbolic conservation equation (Eq. (1)), we consider a general numerical scheme of the following form:

$$
q_{i}^{n+1}=q_{i}^{n}-C_{i-1 / 2}\left(q_{i}^{n}-q_{i-1}^{n}\right)+D_{i+1 / 2}\left(q_{i+1}^{n}-q_{i}^{n}\right)
$$

where $C_{i-1 / 2}$ and $D_{i+1 / 2}$ are data dependent coefficients. Harten [29, 33] has proved that the sufficient conditions for this scheme to be TVD are the following inequalities:

$$
C_{i+1 / 2} \geq 0, \quad D_{i+1 / 2} \geq 0, \quad 0 \leq C_{i+1 / 2}+D_{i+1 / 2} \leq 1
$$

Substituting the usual explicit flux-limited form (Eq. (7)) into the general explicit fluxconservative form (Eq. (4)), the following equation is derived: 


$$
\begin{aligned}
q_{i}^{n+1} & =q_{i}^{n}-v \cdot\left(\left[q_{i}^{n}+\Phi\left(r_{i+1 / 2}\right) \frac{(1-v)}{2}\left(q_{i+1}^{n}-q_{i}^{n}\right)\right]-\left[q_{i-1}^{n}+\Phi\left(r_{i-1 / 2}\right) \frac{(1-v)}{2}\left(q_{i}^{n}-q_{i-1}^{n}\right)\right]\right) \\
& =q_{i}^{n}-v \cdot\left(q_{i}^{n}-q_{i-1}^{n}\right)-\frac{1}{2} v(1-v)\left(\Phi\left(r_{i+1 / 2}\right) \cdot\left(q_{i+1}^{n}-q_{i}^{n}\right)-\Phi\left(r_{i-1 / 2}\right) \cdot\left(q_{i}^{n}-q_{i-1}^{n}\right)\right) \\
& =q_{i}^{n}-\left[v+\frac{1}{2} v(1-v)\left(\frac{\Phi\left(r_{i+1 / 2}\right)}{r_{i+1 / 2}}-\Phi\left(r_{i-1 / 2}\right)\right)\right]\left(q_{i}^{n}-q_{i-1}^{n}\right)
\end{aligned}
$$

Obviously, Eq. (15) has the same form as the Eq. (13) with

$$
\begin{aligned}
\mathrm{C}_{i-1 / 2} & =\left[v+\frac{1}{2} v(1-v)\left(\frac{\Phi\left(r_{i+1 / 2}\right)}{r_{i+1 / 2}}-\Phi\left(r_{i-1 / 2}\right)\right)\right] \\
D_{i+1 / 2} & =0
\end{aligned}
$$

Substituting Eq. (16) into Eq. (14) results in the following limiting condition:

$$
0 \leq v+\frac{1}{2} v(1-v)\left(\frac{\Phi\left(r_{i+1 / 2}\right)}{r_{i+1 / 2}}-\Phi\left(r_{i-1 / 2}\right)\right) \leq 1
$$

which is equivalent to

$$
-\frac{2}{1-v} \leq\left(\frac{\Phi\left(r_{i+1 / 2}\right)}{r_{i+1 / 2}}-\Phi\left(r_{i-1 / 2}\right)\right) \leq \frac{2}{v}
$$

In addition, for negative $r_{i+1 / 2}$ values, $\Phi\left(r_{i+1 / 2}\right)$ is usually set to zero.

$$
\Phi\left(r_{i+1 / 2}\right)=0 \quad \text { when } r_{i+1 / 2} \leq 0
$$

Although most flux-limiters proposed so far satisfy the above condition (Eq. (19)), the violation of this condition does not cause problems in practice. This has been illustrated in schemes such as the OSPRE limiter, the Albada family limiters, the variant Arora-Roe limiter, the local double-logarithmic reconstruction (LDLR) limiter and the Čada-Torrilhon limiter [10, 11, 33, 39]. In fact, the TCDF limiter, newlyproposed in this paper, also does not satisfy this condition, which will be described in details later on. In the present stage, however, we restrict ourselves to the construction of the general limiting criterion for OTU-TVD schemes. Hence, as usual, by combining Eq. (18) with Eq. (19), the One-step Unsteady TVD criterion is defined as:

$$
\begin{cases}0 \leq \Phi\left(r_{i+1 / 2}\right) \leq \min \left(\frac{2}{1-v}, \frac{2 r_{i+1 / 2}}{v}\right) & \text { when } r_{i+1 / 2}>0 \\ 0 & \text { when } r_{i+1 / 2} \leq 0\end{cases}
$$

The above criterion is illustrated by the shaded region in Figure 1. It is apparent that this CFL-dependent TVD region, firstly conceived by Roe [44, 51], is not bounded by $\left[2,2 r_{i+1 / 2}\right]$ and is thus different from the well-known Sweby's TVD region [30], 
which is also referred to as the Semi-discrete Steady-state TVD criterion in this paper and will be discussed in the next section.

To avoid confusion, we emphasize that the retaining of the CFL-number as a parameter in the criterion is simply because the One-step Unsteady TVD criterion is designed for unsteady analysis of the 1D advection equation. This is not necessary when a steady-state solution is sought after, as pointed out by Arora and Roe [44]. Instead the Semi-discrete Steady-state TVD criterion (viz. Sweby's TVD criterion) should be employed under such a circumstance.

\subsection{OTU-TVD Flux-limiters}

As mentioned earlier, OTU-TVD schemes can be expressed by the usual explicit flux-limited form (Eq. (7)), in which the limiter function $\left(\Phi\left(r_{i+1 / 2}\right)\right)$ determines the accuracy of the scheme. In order to suppress spurious oscillations in the vicinity of steep gradients, $\Phi\left(r_{i+1 / 2}\right)$ is required to satisfy the One-step Unsteady TVD criterion (Eq. (20)), and consequently be confined to the shaded region shown in Figure 1.

Although abundant CFL-independent flux-limiters have been developed in the past decades for solving steady-state problems, only a small number of publications have been dedicated to the construction of OTU-TVD flux-limiters in the context of one-step unsteady computations, which retain the dependence on the CFL-number and are required to satisfy the One-step Unsteady TVD criterion. Some of the flux-limiters that have been used are listed below:

Arora-Roe limiter [10, 33, 34, 44, 51], also known as the Direct Scheme in [32] and referred to as CFL-Koren limiter in this paper:

$$
\Phi\left(r_{i+1},\right)=\max \left\{0, \min \left\{\frac{2}{1-v}, 1-\frac{1+v}{3}\left(1-r_{i+}\right), \frac{2 r_{i+1} /}{v}\right\}\right\}
$$

Adaptive-QUICK limiter [53], also called as CFL-WACEB limiter in this paper:

$$
\Phi\left(r_{i+1 / 2}\right)=\max \left\{0, \min \left\{\frac{2}{1-v}, \frac{1}{4} r_{i+1 / 2}+\frac{3}{4}, \frac{2 r_{i+1 / 2}}{v}\right\}\right\}
$$

CFL-Superbee limiter [33]:

$$
\Phi\left(r_{i+1 / 2}\right)=\max \left\{0, \min \left\{\frac{2}{1-v}, \max \left(1, r_{i+1 / 2}\right), \frac{2 r_{i+1 / 2}}{v}\right\}\right\}
$$

CFL-MUSCL limiter [31, 33]: 


$$
\Phi\left(r_{i+1 / 2}\right)=\max \left\{0, \min \left\{\frac{2}{1-v}, \frac{1}{2} r_{i+1 / 2}+\frac{1}{2}, \frac{2 r_{i+1 / 2}}{v}\right\}\right\}
$$

Modified CFL-Superbee limiter [33]:

$\Phi\left(r_{i+1 / 2}\right)=\max \left\{0, \min \left\{\frac{2}{1-v}, \max \left\{1+\left(\frac{1+v}{3}-\frac{\beta}{2}\right)\left(r_{i+1 / 2}-1\right), 1+\left(\frac{1+v}{3}+\frac{\beta}{2}\right)\left(r_{i+1 / 2}-1\right)\right\}, \frac{2 r_{i+1 / 2}}{v}\right\}\right\}$

(where $\beta$ is a parameter $0 \leq \beta \leq 2 / 3$ )

Hyperbee limiter [33, 51]:

$$
\Phi\left(r_{i+1 / 2}\right)= \begin{cases}0 & r_{i+1 / 2} \leq 0 \\ 1 & r_{i+1 / 2}=1 \\ \frac{2 r_{i+1 / 2}}{v(1-v)} \cdot \frac{v\left(r_{i+1 / 2}-1\right)+\left[1-\left(r_{i+1 / 2}\right)^{v}\right]}{\left(r_{i+1 / 2}-1\right)^{2}} & \text { otherwise }\end{cases}
$$

Superpower limiter [33]:

$$
\begin{aligned}
& \Phi\left(r_{i+1 / 2}\right)=\max \left\{0,\left[1-\frac{1+v}{3}\left(1-r_{i+1 / 2}\right)\right] \cdot\left(1-\left|\frac{1-\left|r_{i+1 / 2}\right|}{1+\left|r_{i+1 / 2}\right|}\right|\right)^{p(r)}\right\} \\
& \text { where } p(r)= \begin{cases}\frac{2}{v} \cdot 2\left[1-\frac{(1+v)}{3}\right] & r_{i+1 / 2} \leq 1 \\
\frac{2}{1-v} \cdot \frac{2(1+v)}{3} & r_{i+1 / 2} \geq 1\end{cases}
\end{aligned}
$$

Variant Arora-Roe limiter [33]:

$\Phi\left(r_{i+1 / 2}\right)=\min \left\{\max \left\{-(1-\beta) \frac{2}{v}, 1-\frac{1+v}{3}\left(1-r_{i+1 / 2}\right)\right\}, \max \left\{-(1-\beta) \frac{2}{1-v} r_{i+1 / 2}, \beta \frac{2}{v} r_{i+1 / 2}\right\}, \beta \frac{2}{1-v}\right\}$

(where $\beta$ is a parameter $1 / 2 \leq \beta \leq 1$ )

The above-listed flux-limiters, from Eq. (21) to Eq. (28), can be basically divided into two types: piecewise-linear and smooth limiters. The piecewise-linear flux-limiters, such as Eqs. (21-25) and Eq. (28), simply switch between different linear schemes, depending on the local gradient ratio $r_{i+1 / 2}$. But, the smooth flux-limiters, such as Eq. (26) and Eq. (27), are constructed with non-linear gradually-switching smooth functions (at least on the positive $r$-axis). Amongst these limiters, Arora-Roe limiter (Eq. (21)) maintains the same third-order accuracy both temporally and spatially, and is one of the most widely used OTU-TVD schemes [10, 32-34, 44, 51], due to its excellent overall performance in terms of accuracy and efficiency. 
In summary, this section focuses on a review of the development of OTU-TVD schemes for solving the 1D unsteady linear advection equation. As previously demonstrated, OTU-TVD schemes adopt the general explicit flux-conservative form (Eq. (4)) to discretize the unsteady advection equation, and further employ the usual explicit flux-limited form (Eq. (7)) to express the conservative flux. It is worthwhile to re-emphasize that different OTU-TVD schemes are distinguished by the form of the limiter function $\Phi\left(r_{i+1 / 2}\right)$ in Eq. (7), which also determines the accuracy of the scheme. The flux-limiter function $\Phi\left(r_{i+1 / 2}\right)$ is required to satisfy the One-step Unsteady TVD criterion which is illustrated by the shaded region in Figure 1.

\section{Semi-Discrete Steady-State TVD Schemes}

This section provides a brief review of various SS-TVD schemes designed for the steady-state solution of the 1D advection equation:

$$
q_{t}=-(a q)_{x}
$$

where $q$ denotes the dependent variable and $a$ is the advection velocity. Without losing generality, the advection velocity is assumed to be positive $(a>0)$ because the opposite case $(a<0)$ can be treated by symmetry. Note that the flux term is deliberately moved to the right-hand side of Eq. (29) to emphasize the semi-discrete nature.

It is trivial to show that obtaining the steady-state solution of Eq. (29) is essentially equivalent to solving the steady equation $(a q)_{x}=0$. Since a pseudo-timestepping approach is employed for various schemes reviewed in this section, we elect to use Eq. (29) as the model equation for steady advection problems. It should be pointed out that all SS-TVD schemes, originally developed for Eq. (29), are equally applicable to solving the boundary value problem $(a q)_{x}=0$, as described in [11, 13-14, 27-28, 36-39, 45-50, 53].

\subsection{Semi-Discrete Steady-State Linear Schemes}

Discretizing Eq. (29) over a control volume $C_{i}=\left[x_{i-\Delta x / 2}, x_{i+\Delta x / 2}\right]$, one can obtain the general semi-discrete flux-conservative form:

$$
\frac{d q_{i}}{d t}=\frac{-a\left(q_{i+1 / 2}-q_{i-1 / 2}\right)}{\Delta x}
$$

In order to predict the variable value at the cell-face, $q_{i+1 / 2}$ or $q_{i-1 / 2}$, the well- 
known $k$-schemes, firstly introduced by Van Leer [11, 54, 55], are employed in consideration of their compact 5-point stencil in each spatial direction. The numerical flux of the $k$-schemes on uniform 1D grids reads:

$$
q_{i+1 / 2}=q_{i}+\left\{\frac{1+k}{4}\left(q_{i+1}-q_{i}\right)+\frac{1-k}{4}\left(q_{i}-q_{i-1}\right)\right\}
$$

which can be extended to non-uniform $1 \mathrm{D}$ grids:

$$
\begin{gathered}
\qquad q_{i+1 / 2}=q_{i}+\frac{\Delta x_{i}}{2}\left\{\frac{1+k}{2}\left(\frac{\partial q}{\partial x}\right)_{i+1 / 2}+\frac{1-k}{2}\left(\frac{\partial q}{\partial x}\right)_{i-1 / 2}\right\} \\
\text { where }\left(\frac{\partial q}{\partial x}\right)_{i+1 / 2}=\frac{q_{i+1}-q_{i}}{x_{i+1}-x_{i}},\left(\frac{\partial q}{\partial x}\right)_{i-1 / 2}=\frac{q_{i}-q_{i-1}}{x_{i}-x_{i-1}}, \Delta x_{i}=x_{i+1 / 2}-x_{i-1 / 2}
\end{gathered}
$$

where $k$ is a variable representing different schemes. Actually, the $k$-schemes can be deemed as a combination of a dissipative FOU scheme (the first term on the right-hand side) and a high-order correction term (the rest of the right-hand side). The high-order correction term employs a linear weighted average of two local gradients: one across the cell face in question $(\partial q / \partial x)_{i+1 / 2}$ and the other across the immediate upwind cell face $(\partial q / \partial x)_{i-1 / 2}$.

As described in [11], it is quite natural to rewrite the above $k$-schemes in the usual semi-discrete flux-limited form on a uniform grid,

$$
\begin{gathered}
q_{i+1 / 2}=q_{i}+\frac{1}{2} \psi\left(\tilde{r}_{i+1 / 2}\right)\left(q_{i}-q_{i-1}\right) \\
\text { where } \psi\left(\tilde{r}_{i+1 / 2}\right)=\frac{1+k}{2} \tilde{r}_{i+1 / 2}+\frac{1-k}{2}, \quad \tilde{r}_{i+1 / 2}=\frac{\left(q_{i+1}-q_{i}\right)}{\left(q_{i}-q_{i-1}\right)}
\end{gathered}
$$

Similarly, Eq. (33) takes the following form on a non-uniform mesh,

$$
\begin{gathered}
q_{i+1 / 2}=q_{i}+\frac{\Delta x_{i}}{2} \psi\left(\tilde{r}_{i+1 / 2}\right)\left(\frac{\partial q}{\partial x}\right)_{i-1 / 2} \\
\text { where } \psi\left(\tilde{r}_{i+1 / 2}\right)=\frac{1+k}{2} \tilde{r}_{i+1 / 2}+\frac{1-k}{2}, \quad \tilde{r}_{i+1 / 2}=\left(\frac{\partial q}{\partial x}\right)_{i+1 / 2} /\left(\frac{\partial q}{\partial x}\right)_{i-1 / 2}
\end{gathered}
$$

Analogous to the usual explicit flux-limited form (Eq. (7)), the flux-limiter $\psi\left(\tilde{r}_{i+1 / 2}\right)$ in the usual semi-discrete flux-limited form (Eq. (33) or Eq. (34)) determines the accuracy of the scheme. Since the interest is on the steady-state solution in this case, we focus on the spatial accuracy only and ignore any time-step restrictions.

Although the semi-discrete flux-limited form can also be formulated in slightly different forms from the one given by Eq. (33) or Eq. (34), as those outlined in [28, 
$36,38,45,53]$, it is possible to convert those different forms and their corresponding flux-limiters into the usual semi-discrete flux-limited form through simple algebraic manipulations. Therefore, the usual semi-discrete flux-limited form (Eq. (33) or Eq. (34)) is the only form considered in this paper for the purpose of consistency.

It is well known that some of the classical linear high-order advection schemes can be derived by taking different values of $k$, e.g.

$$
\begin{array}{ll}
\operatorname{SOU}(k=-1): & \psi\left(\tilde{r}_{i+1 / 2}\right)=1 \\
\text { Fromm }(k=0): & \psi\left(\tilde{r}_{i+1 / 2}\right)=\left(\tilde{r}_{i+1 / 2}+1\right) / 2 \\
\text { CUI }(k=1 / 3): & \psi\left(\tilde{r}_{i+1 / 2}\right)=\left(2 \tilde{r}_{i+1 / 2}+1\right) / 3 \\
\text { QUICK }(k=1 / 2): & \psi\left(\tilde{r}_{i+1 / 2}\right)=\left(3 \tilde{r}_{i+1 / 2}+1\right) / 4 \\
\text { CDS }(k=1): & \psi\left(\tilde{r}_{i+1 / 2}\right)=\tilde{r}_{i+1 / 2}
\end{array}
$$

It is equally well known that linear high-order advection schemes, such as members of the $k$-schemes (Eq. (35)), are vulnerable to unphysical spatial oscillations when they are applied to capture shocks or steep variations of the dependent variable, primarily due to their unbounded nature [7-9].

\subsection{Semi-Discrete Steady-State TVD Criterion}

In order to achieve the desired boundedness property for the usual semi-discrete flux-limited form (Eq. (33) or Eq. (34)), several boundedness criteria have been proposed in the literature, such as Sweby's TVD criterion [11, 30], Gaskell's CBC criterion $[25,26]$ and Spekreijse's Positivity criterion $[14,56]$.

Sweby's TVD criterion was originally deduced in the context of an explicit time discretization, but has been broadly applied to the construction of semi-discrete steady-state CFL-independent flux-limiters [11, 13-14, 27-28, 30, 36-39, 45-50, 55-56]. In this section, Sweby's TVD criterion is adopted to construct non-linear monotonicity-preserving SS-TVD schemes in order to avoid spurious oscillations in the vicinity of discontinuities or steep gradients.

Following the same approach outlined in Subsection 2.2, it is easy to derive the Semi-discrete Steady-state TVD criterion (viz. Sweby's TVD criterion), which is also illustrated in Figure 2:

$$
\begin{cases}0 \leq \psi\left(\tilde{r}_{i+1 / 2}\right) \leq \min \left(2 \tilde{r}_{i+1 / 2}, 2\right) & \text { when } \tilde{r}_{i+1 / 2}>0 \\ \psi\left(\tilde{r}_{i+1 / 2}\right)=0 & \text { when } \tilde{r}_{i+1 / 2} \leq 0\end{cases}
$$


As previously stated, contrary to the One-step Unsteady TVD criterion (Eq. (20)), the Semi-discrete Steady-state TVD criterion (Eq. (36)) gets rid of the dependence on the CFL number, which is believed to be advisable for solving the steady-state solution of the semi-discrete advection equation (Eq. (29)) by using a pseudotime-stepping approach.

\subsection{SS-TVD Flux-Limiters}

In the past decades, a large number of SS-TVD flux-limiters that satisfy the Semi-discrete Steady-state TVD criterion (Eq. (36)) have been proposed and analyzed. Some of those limiters are listed below for the convenience of discussion.

1). Piecewise-linear SS-TVD Flux-limiters:

Chakravarthy and Osher limiter [11, 30]:

$$
\psi\left(\tilde{r}_{i+1 / 2}\right)=\operatorname{ma}\left\{0, \mathrm{mi}\left(\tilde{r}_{i+1 / 2}, \beta\right)\right], \text { where } 1 \leq \beta \leq 2
$$

BSOU limiter $[4,6,11,30]$ :

$$
\psi\left(\tilde{r}_{i+1 / 2}\right)=\operatorname{ma}\left[0, \mathrm{~m} \mathrm{i}\left(2 \tilde{r}_{i+1 / 2}, 1\right)\right]
$$

Minmod limiter [4, 6, 11-13, 15, 30, 42, 47]:

$$
\psi\left(\tilde{r}_{i+1 / 2}\right)=\operatorname{ma}\left\{0, \mathrm{~m} \text { i }\left(\tilde{r}_{i+1 / 2}, 1\right)\right]
$$

Superbee limiter $[2,4,6,11-13,15,30,33,42,47]$ :

$$
\psi\left(\tilde{r}_{i+1 / 2}\right)=\mathrm{m} \text { a }\left[0, \mathrm{mi}\left(2 \tilde{r}_{i+1 / 2}, 1\right), \mathrm{mi}\left(\tilde{r}_{i+1 / 2}, 2\right)\right]
$$

MUSCL limiter [2, 4, 6, 11-14, 30, 31, 33, 42]:

$$
\psi\left(\tilde{r}_{i+1 / 2}\right)=\mathrm{m} \text { a }\left[\mathrm{x} 0, \mathrm{mi}\left(2 \tilde{r}_{i+1 / 2}, \frac{\tilde{r}_{i+1 / 2}+1}{2}, 2\right)\right]
$$

Koren limiter [4, 6, 11, 32, 33, 39, 49, 50, 55]:

$$
\psi\left(\tilde{r}_{i+1 / 2}\right)=\mathrm{m} \text { a }\left[\mathrm{x} 0, \mathrm{mi}\left(2 \tilde{r}_{i+1 / 2}, \frac{2 \tilde{r}_{i+1 / 2}+1}{3}, 2\right)\right]
$$

WACEB limiter [4, 6, 11, 23], also known as the Bounded QUICK [48, 53]:

$$
\psi\left(\tilde{r}_{i+1 / 2}\right)=\max \left[0, \mathrm{mi}\left(2 \tilde{r}_{i+1 / 2}, \frac{3 \tilde{r}_{i+1 / 2}+1}{4}, 2\right)\right]
$$

UMIST limiter [4, 48], also known as the SPL-1/2 limiter [11]:

$$
\psi\left(\tilde{r}_{i+1 / 2}\right)=\max \left[0, \mathrm{~m} \text { i }\left(22 \tilde{r}_{i+1 / 2}, \frac{3 \tilde{r}_{i+1 / 2}+1}{4}, \frac{\tilde{r}_{i+1 / 2}+3}{4}, 2\right)\right]
$$


SPL-max-1/2 limiter [11]:

$$
\left.\psi\left(\tilde{r}_{i+1 / 2}\right)=\max 0, \mathrm{mi}\left(2 \tilde{r}_{i+1 / 2}, \mathrm{ma}\left(\frac{3 \tilde{r}_{i+1 / 2}+1}{4}, \frac{\tilde{r}_{i+1 / 2}+3}{4}\right), 2\right)\right]
$$

SPL-1/3 limiter [11]:

$$
\psi\left(\tilde{r}_{i+1 / 2}\right)=\mathrm{m} \text { a }\left[\mathrm{x} 0, \mathrm{mi}\left(2 \tilde{r}_{i+1 / 2}, \frac{2 \tilde{r}_{i+1 / 2}+1}{3}, \frac{\tilde{r}_{i+1 / 2}+2}{3}, 2\right)\right]
$$

SPL-max-1/3 limiter [11]:

$$
\left.\psi\left(\tilde{r}_{i+1 / 2}\right)=\max 0, \mathrm{mi}\left(2 \tilde{r}_{i+1 / 2}, \mathrm{~m} \mathrm{a}\left(\frac{2 \tilde{r}_{i+1 / 2}+1}{3}, \frac{\tilde{r}_{i+1 / 2}+2}{3}\right), 2\right)\right]
$$

\section{2). Smooth SS-TVD Flux-limiters:}

Harmonic limiter [4, 11, 12, 30, 31, 33, 42, 47, 50]:

$$
\psi\left(\tilde{r}_{i+1 / 2}\right)=\frac{\tilde{r}_{i+1 / 2}+\left|\tilde{r}_{i+1 / 2}\right|}{\tilde{r}_{i+1 / 2}+1}
$$

OSPRE limiter [4, 11, 33, 47]:

$$
\psi\left(\tilde{r}_{i+1 / 2}\right)=\frac{3 \tilde{r}_{i+1 / 2}\left(\tilde{r}_{i+1 / 2}+1\right)}{2\left[\left(\tilde{r}_{i+1 / 2}\right)^{2}+\tilde{r}_{i+1 / 2}+1\right]}
$$

Albada limiter [4, 11, 14, 33, 57], also known as the GVA-0 limiter [11]:

$$
\psi\left(\tilde{r}_{i+1 / 2}\right)=\frac{\tilde{r}_{i+1 / 2}\left(\tilde{r}_{i+1 / 2}+1\right)}{\left(\tilde{r}_{i+1 / 2}\right)^{2}+1}
$$

GVA-1/3 limiter [11]:

$$
\psi\left(\tilde{r}_{i+1 / 2}\right)=\frac{\tilde{r}_{i+1 / 2}\left(\tilde{r}_{i+1 / 2}+2\right)}{\left(\tilde{r}_{i+1 / 2}\right)^{2}+2}
$$

GVA-1/2 limiter [11]:

$$
\psi\left(\tilde{r}_{i+1 / 2}\right)=\frac{\tilde{r}_{i+1 / 2}\left(\tilde{r}_{i+1 / 2}+3\right)}{\left(\tilde{r}_{i+1 / 2}\right)^{2}+3}
$$

GPR-0 limiter [11]:

$$
\psi\left(\tilde{r}_{i+1 / 2}\right)=\frac{\tilde{r}_{i+1 / 2}\left(3 \tilde{r}_{i+1 / 2}+1\right)}{2\left(\tilde{r}_{i+1 / 2}\right)^{2}+\tilde{r}_{i+1 / 2}+1}
$$

GPR-1/3 limiter [11]:

$$
\psi\left(\tilde{r}_{i+1 / 2}\right)=\frac{\tilde{r}_{i+1 / 2}\left(2 \tilde{r}_{i+1 / 2}+1\right)}{\left(\tilde{r}_{i+1 / 2}\right)^{2}+\tilde{r}_{i+1 / 2}+1}
$$

GPR-1/2 limiter [11]: 


$$
\psi\left(\tilde{r}_{i+1 / 2}\right)=\frac{2 \tilde{r}_{i+1 / 2}\left(\tilde{r}_{i+1 / 2}+1\right)}{\left(\tilde{r}_{i+1 / 2}\right)^{2}+\tilde{r}_{i+1 / 2}+2}
$$

As a matter of fact, many flux-limiters of this kind, which are not listed in this study, can be found in the literature. Interested readers are referred to [10-11, 28, 38-39, 42]. Here, we only present several representatives of the piecewise-linear flux-limiters and gradually-switching smooth flux-limiters for the convenience of discussions. Generally speaking, the piecewise-linear flux-limiters (e.g. Eqs. (37-47)) act simply as switches between different linear schemes and offer the advantage of great flexibility. The overall accuracy of the piecewise-linear flux-limiters can be improved by enlarging the region of a specified higher-order scheme (e.g. QUICK or CUI). However, the flux-limiters of this kind suffer from an adverse effect on convergence behavior under some circumstances because of their discontinuous nature. In contrast, the gradually-switching smooth flux-limiters (e.g. Eqs. (48-55)) exhibit better convergence behavior than the piecewise-linear flux-limiters at a price of accuracy [11]. This motivates the present study to develop a refined CFLindependent gradually-switching smooth flux-limiter (TCDF) that simultaneously maintains the advantage of the piecewise-linear flux-limiters for steady-state calculations within the SS-TVD framework.

In summary, this section provides a brief review of various SS-TVD schemes designed for the steady-state solution of the 1D advection equation. The key elements of the SS-TVD schemes include the general semi-discrete flux-conservative form (Eq. (30)) to discretize the advection term and the usual semi-discrete flux-limited form, Eq. (33) or Eq. (34), to approximate the conservative flux. It is the CFL-independent limiter $\psi\left(\tilde{r}_{i+1 / 2}\right)$ that distinguishes different SS-TVD schemes from each other and determines the accuracy of the scheme.

\section{Multi-Step Time-Space-Separated Unsteady TVD Schemes}

As discussed by Daru and Tenaud [10], the MTU-TVD schemes follow the methodof-lines approach $[32,55]$ by implementing the spatial and temporal discretizations separately. Furthermore, these schemes employ Runge-Kutta type time-stepping methods rather than the Taylor series expansion approach for the temporal discretization. A widely-used third-order TVD RK3 scheme [10, 32, 55, 58-60] is outlined to explain the fundamental philosophy of the MTU-TVD schemes: 


$$
\left\{\begin{array}{l}
q_{i}^{1}=q_{i}^{n}+\Delta t \cdot L_{\Delta x}\left(q_{i}^{n}\right) \\
q_{i}^{2}=\frac{3}{4} q_{i}^{n}+\frac{1}{4} q_{i}^{1}+\frac{\Delta t}{4} \cdot L_{\Delta x}\left(q_{i}^{1}\right) \\
q_{i}^{n+1}=\frac{1}{3} q_{i}^{n}+\frac{2}{3} q_{i}^{2}+\frac{2 \Delta t}{3} \cdot L_{\Delta x}\left(q_{i}^{2}\right)
\end{array}\right.
$$

where $L_{\Delta x}\left(q_{i}\right)$ is the spatial discretization operator, defined as:

$$
L_{\Delta x}\left(q_{i}\right)=\frac{-a\left(q_{i+1 / 2}-q_{i-1 / 2}\right)}{\Delta x}
$$

It is seen from Eq. (56) that a multi-stage Runge-Kutta method is made up of repeated applications of a single-stage scheme. Therefore, once $L_{\Delta x}\left(q_{i}\right)$ is determined, the description of a Runge-Kutta method is completed.

To ensure the TVD property of a multistage scheme in a strict sense, not all Runge-Kutta schemes can be directly used to deal with the time discretization because both the Runge-Kutta solver and each space-discretization step of the solver are required to be TVD. For this reason, this section is limited to the construction of high-resolution TVD schemes for each space-discretization step $\left(L_{\Delta x}\left(q_{i}\right)\right)$ only, and ignores the problem of choosing different Runge-Kutta schemes.

\subsection{Multi-Step Time-Space-Separated Spatial Linear Schemes}

As aforementioned, in order to assure the TVD property of a multistage scheme, we must make each space-discretization step $\left(L_{\Delta x}\left(q_{i}\right)\right)$ of the Runge-Kutta solver to be TVD. It is apparent that $L_{\Delta x}\left(q_{i}\right)$ is equal to the right-hand side of the general semi-discrete flux-conservative form (Eq. (30)).

As discussed in Subsection 3.1, the well-known $k$-schemes are adopted to predict the convected variable value at the cell-face, $q_{i+1 / 2}$ or $q_{i-1 / 2}$, in consideration of their compact 5-point stencil in each spatial direction. Similarly, the usual semi-discrete flux-limited form (Eq. (33) or Eq. (34)) are employed, and the flux-limiter $\psi\left(\tilde{r}_{i+1 / 2}\right)$ determines the accuracy of the scheme. Members of the $k$-schemes can be expressed with different linear flux-limiters, as demonstrated in Eq. (35).

\subsection{Multi-Step Unsteady TVD Criterion}

In Subsection 3.2, the Semi-discrete Steady-state TVD criterion, viz. Sweby's TVD criterion given by Eq. (36), is used to achieve the desired TVD property for the usual 
semi-discrete flux-limited form (Eq. (33) or Eq. (34)). Although Sweby's TVD criterion works well for obtaining the steady-state solution of the semi-discrete advection equation (Eq. (29)), it is not suitable for the multi-step unsteady computations. A specific TVD criterion is needed under this circumstance.

Through simple algebraic manipulations, Eq. (33) can be converted into the following form:

$$
q_{i+1 / 2}=q_{i}+\frac{1}{2} \tilde{\Phi}\left(r_{i+1 / 2}\right)\left(q_{i+1}-q_{i}\right)
$$

where the gradient ratio $r_{i+1 / 2}$ is defined as $r_{i+1 / 2}=1 / \tilde{r}_{i+1 / 2}=\left(q_{i}-q_{i-1}\right) /\left(q_{i+1}-q_{i}\right)$, the same as that defined in Eq. (8). $\tilde{\Phi}\left(r_{i+1 / 2}\right)$ is a new flux-limiter function slightly different from $\psi\left(\tilde{r}_{i+1 / 2}\right)$ in Eq. (33). The two functions are related in the following manner [45]:

$$
\tilde{\Phi}\left(r_{i+1 / 2}\right)=\frac{1}{\tilde{r}_{i+1 / 2}} \psi\left(\tilde{r}_{i+1 / 2}\right)
$$

For a multi-stage Runge-Kutta method, each sub-step of the time integration is essentially equivalent to explicitly solving the unsteady advection equation (Eq. (1)). So it is reasonable for us to transform the MTU-TVD flux-limited form (Eq. (58)) into the usual explicit flux-limited form (Eq. (7)), aiming at deriving the conditions to ensure the TVD property in each sub-step of the time integration. Hence, we further rewrite Eq. (58) as:

$$
q_{i+1 / 2}=q_{i}+\frac{\tilde{\Phi}\left(r_{i+1 / 2}\right)}{1-v} \frac{(1-v)}{2}\left(q_{i+1}-q_{i}\right)
$$

Obviously, differences do exist between OTU-TVD schemes and MTU-TVD schemes. In the former, the spatial discretization is completely coupled with the temporal discretization, so the usual explicit flux-limited form (Eq. (7)) for the space reconstruction is CFL-dependent. In the latter, the spatial discretization is absolutely independent of the time integration, thus the MTU-TVD flux-limited form (Eq. (58) or Eq. (60)) is essentially CFL-independent.

Replacing Eq. (7) by Eq. (60) and following the same derivation process outlined in Subsection 2.2, we can obtain the following Multi-step Unsteady TVD criterion [10], which is also illustrated graphically in Figure 3:

$$
\left\{\begin{array}{ll}
0 \leq \frac{\tilde{\Phi}\left(r_{i+1 / 2}\right)}{1-v} \leq \min \left(\frac{2}{1-v}, \frac{2 r_{i+1 / 2}}{v}\right) & \text { when } r_{i+1 / 2}>0 \\
0 & \text { when } r_{i+1 / 2} \leq 0
\end{array} \Rightarrow\right.
$$




$$
\begin{cases}0 \leq \tilde{\Phi}\left(r_{i+1 / 2}\right) \leq \min \left(2, \frac{(1-v) \cdot 2 r_{i+1 / 2}}{v}\right) & \text { when } r_{i+1 / 2}>0 \\ 0 & \text { when } r_{i+1 / 2} \leq 0\end{cases}
$$

In order to keep at least second-order accuracy in smooth regions, it is necessary that $\tilde{\Phi}(1)=1$, which implies the following CFL condition:

$$
1 \leq \frac{(1-v) \cdot 2}{v} \Rightarrow v \leq \frac{2}{3}
$$

From Eq. (36) and Eq. (61), it can be concluded that the MTU-TVD criterion is more restrictive than the SS-TVD criterion when the CFL number lies in the range of $(0.5,1.0]$. However, the former is less restrictive than the latter for the CFL values lower than 0.5. Specifically, when the CFL number is equal to 0.5, the former (Eq. (61)) is essentially equal to the latter (viz. the Sweby's TVD criterion, Eq. (36)). In other words, for CFL-numbers lower than or equal to 0.5, the SS-TVD criterion is the most demanding situation of the MTU-TVD criterion [10].

We would like to re-emphasize that, for the MTU-TVD schemes, the spatial discretization is absolutely independent of the time integration. Therefore, we adopt the CFL-independent flux-limited form (Eq. (58) or Eq. (60)) for the space reconstruction. In order to ensure the TVD property in each sub-step of the time integration of the Runge-Kutta solver, time-step restrictions need to be reintroduced. This consequently results in the Multi-step Unsteady TVD criterion being CFL-dependent (Eq. (61)).

\subsection{MTU-TVD Flux-Limiters}

Theoretically speaking, various piecewise-linear MTU-TVD flux-limiters can be obtained by simply replacing the Semi-discrete Steady-state TVD criterion (Eq. (36)) with the Multi-step Unsteady TVD criterion (Eq. (61)) in Eqs. (40-47). Furthermore, different smooth MTU-TVD flux-limiters can also be formulated by adopting the upper bound value of Eq. (61), viz. $\max \left\{0, \min \left(2,2 r_{i+1 / 2} \cdot(1-v) / v\right)\right\}$, rather than $\max \left\{0, \min \left(2,2 r_{i+1 / 2}\right)\right\}$ specified by Eq. (36).

In practice, either the OTU-TVD flux-limiters (Eqs. (21-28)) or the SS-TVD fluxlimiters (Eqs. (37-55)) have been used in each sub-step of the time integration of the Runge-Kutta solver $[10,32,55,60]$. It should be pointed out that the MTU-TVD criterion (Eq. (61)) is more restrictive than the OTU-TVD criterion (Eq. (20)). Besides, the use of the OTU-TVD flux-limiters in this situation is not time-efficient, 
because the temporal accuracy has already been guaranteed by the Runge-Kutta solver. In addition, it is also not all that sensible to adopt the SS-TVD flux-limiters at this moment because the Semi-discrete Steady-state TVD criterion (Eq. (36)) is excessively restrictive (relative to Eq. (61)) for the CFL values lower than 0.5.

Although the MTU-TVD flux-limiters can be classified as one of the basic flux-limiter types, it is not our intention to propose new schemes of this kind. Therefore they are not compared with other kinds of flux-limiters quantitatively here. We would like to maintain our focus on presenting a detailed classification of TVD schemes and developing a refined SS-TVD flux-limiter for steady-state calculations.

\subsection{Relationships between Different TVD limiters}

All the three kinds of TVD limiters, including the OTU-TVD limiters (Eqs. (21-28)), the SS-TVD limiters (Eqs. (37-55)) and the potential MTU-TVD limiters (designed according to Eq. (61)), are closely related to each other, but distinct differences do exist between them, as discussed in the following paragraphs.

Firstly, it deserves our attention that both the OTU-TVD and the MTU-TVD limiters are developed in the context of unsteady calculations with the purpose of obtaining both the temporal and spatial accuracy. Although the OTU-TVD schemes achieve the temporal and spatial accuracy automatically, the MTU-TVD limiters ensure the temporal accuracy with the aid of the multi-stage Runge-Kutta solver. Furthermore, it should be noted that, for both the OTU-TVD criterion and the MTU-TVD criterion, the CFL number is required to be in the range of $[0,1]$, as indicated by Eq. (20) and Eq. (61).

Secondly, since the SS-TVD flux-limiters are originally proposed for the steady-state solution of the advection equation, the focus of these flux-limiters is mainly on the accuracy of the spatial discretization. It is obvious that these limiters are CFL-independent, as demonstrated by Eqs. (37-55), and therefore larger CFL numbers (than 1.0) can be adopted for the SS-TVD limiters, which are particularly useful when searching for the steady-state solutions.

Thirdly, in theory, when the CFL values lie in the range of $[0,1]$, both the OTU-TVD and SS-TVD limiters can be employed to solve the unsteady advection equation, but we have reasons to believe that the former is likely to result in better numerical accuracy than the latter in this circumstance, because the former maintains both the temporal and spatial accuracy. This will be demonstrated by the numerical 
results presented in the seventh test case of this paper later on. For CFL values larger than 1.0, the OTU-TVD limiters are physically meaningless, but the SS-TVD limiters are still applicable. In other words, the former provide better accuracy for CFL numbers lower than 1.0, whereas, the latter have a greater scope of application in terms of the CFL values.

Additionally, as aforementioned, the MTU-TVD criterion (Eq. (61)) is more restrictive than the OTU-TVD criterion (Eq. (20)), and the SS-TVD criterion (Eq. (36)) is more restrictive than the MTU-TVD criterion when the CFL value is lower than 0.5. But, for CFL numbers in the range of $(0.5,1.0)$, the SS-TVD criterion is actually less restrictive than the MTU-TVD criterion. More importantly, when the CFL number is equal to 0.5 , the former (viz. the Sweby's TVD criterion) is equivalent to the latter. In other words, for CFL-numbers lower than or equal to 0.5, the SS-TVD criterion is the most restrictive criterion.

\subsection{Other Miscellaneous TVD Schemes}

Most TVD flux-limiters proposed so far can be grouped into the aforementioned three broad categories. Nevertheless, a number of miscellaneous flux-limiters, which do not fit into the above classifications for various reasons, do exist in the literature, such as those in $[34,39,40,42,47,61-62]$. To keep the presentation concise, those limiters will not be reviewed here. Interested readers are advised to refer to the relevant references.

\section{A Refined Semi-Discrete Steady-State Flux-Limiter}

In this section, a refined SS-TVD flux-limiter (TCDF) for the steady-state solution of the $1 \mathrm{D}$ advection equation (Eq. (29)) is proposed based on a pseudo-time stepping approach. The newly-proposed TCDF, through maximizing the region of a wellbehaved linear scheme (viz. third-order QUICK) and simultaneously employing a continuously-switching smooth function on the positive $r$-axis, has a better overall performance than the existing SS-TVD flux-limiters in terms of accuracy and convergence.

The general semi-discrete flux-conservative form (Eq. (30)) is employed for the construction of the TCDF, where the flux is estimated by the usual semi-discrete flux-limited form (Eq. (33) for uniform meshes or Eq. (34) for non-uniform meshes). 
Additionally, in order to achieve the desired monotonicity property, the Semi-discrete Steady-state TVD criterion (viz. Sweby's TVD criterion, (Eq. (36)) is adopted for the TCDF, which is described in detail below.

\subsection{On the Positive $r$-axis}

For steady flow computations, Leonard's QUICK scheme [63] is almost the most popular high-order convection scheme. QUICK is an upwind-biased scheme and of third-order accuracy on uniform grids. As demonstrated by Table-B1 in Ref. [11], among all linear $k$-schemes, QUICK shows the highest level of accuracy in the two steady 2D advection test cases. Although handling well the convective transport in problems with smoothly-varying dependent variables, it inevitably generates spurious oscillations in the vicinity of steep-gradients or discontinuities [7, 9, 35, 48, 53, 64].

On the basis of QUICK, a number of high-resolution schemes, such as SHARP [35], SMART [26], AVL-SMART [37], CUBISTA [19], STOTIC [24] and VONOS [65], have been proposed in the normalized variable (NV) diagram to overcome the above defect by employing Gaskell's CBC criterion [25, 26]. In view of the fact that CBC is weaker than TVD [11], these NVD schemes, though performing well in linear advection problems, are not completely bounded in non-linear situations such as shock-tube flows. In addition, originating from QUICK, several piecewise-linear SS-TVD schemes, which strictly satisfy Sweby's TVD criterion, are also developed in the literature, such as WACEB [4, 6, 11, 23] (also known as the Bounded QUICK [48, 53, 64]), UMIST [4, 48] (also known as the SPL-1/2 [11]) and SPL-max-1/2 [11]. It should be noted that, despite offering the advantage of great flexibility and increasing the overall accuracy to some degree by enlarging the region of a specified higher-order scheme (e.g. QUICK), the piecewise-linear TVD schemes suffer from an adverse effect on convergence behavior because of their discontinuous nature. For instance, numerical comparisons in Ref. [11] do reveal that the piecewise-linear SMART scheme, by maximizing the region of QUICK comparatively, indeed leads to the most accurate predictions, but possesses a poor convergence behavior because of its discontinuous nature. On the contrary, continuously-switching smooth limiters, such as OSPRE and Albada, perform very well in terms of convergence behavior.

To ensure both accuracy and convergence, a CFL-independent flux-limiter (TCDF) is delicately designed here within the SS-TVD framework. On the one hand, TCDF coincides with the QUICK scheme to a great degree in smooth regions to achieve 
higher order accuracy. On the other hand, TCDF makes use of a gradually-switching smooth function (at least on the positive $r$-axis), which behaves much better than most of piecewise-linear flux-limiters in terms of convergence.

Firstly, in TCDF, we stick to the third-order QUICK scheme over the interval $0.5 \leq \tilde{r}_{i+1 / 2} \leq 2.0$, which corresponds to the relatively smooth regions. Thus,

$$
\psi\left(\tilde{r}_{i+1 / 2}\right)=0.75 \tilde{r}_{i+1 / 2}+0.25 \quad \text { when } 0.5 \leq \tilde{r}_{i+1 / 2} \leq 2.0
$$

Secondly, in order to obtain a smooth function, a cubic profile is selected to represent this flux-limiter in the region of $0 \leq \tilde{r}_{i+1 / 2}<0.5$ :

$$
\psi\left(\tilde{r}_{i+1 / 2}\right)=a\left(\tilde{r}_{i+1 / 2}\right)^{3}+b\left(\tilde{r}_{i+1 / 2}\right)^{2}+c \tilde{r}_{i+1 / 2}+d
$$

which passes through two points: $(0,0)$ and $(0.5,0.625)$, and is subjected to the gradient conditions: $\psi^{\prime}(0.5)=0.75$ and $\psi^{\prime}(0)=2.0$. This result in

$$
\psi\left(\tilde{r}_{i+1 / 2}\right)=\left(\tilde{r}_{i+1 / 2}\right)^{3}-2\left(\tilde{r}_{i+1 / 2}\right)^{2}+2 \tilde{r}_{i+1 / 2} \quad \text { when } 0 \leq \tilde{r}_{i+1 / 2} \leq 0.5
$$

Additionally, in order to construct gradually-switching smooth limiter function, for the region of $2.0 \leq \tilde{r}_{i+1 / 2}<+\infty$, the general polynomial-ratio (PR) expression of power $n=2$ is utilized:

$$
\psi\left(\tilde{r}_{i+1 / 2}\right)=\frac{M\left(\tilde{r}_{i+1 / 2}\right)^{2}+b_{1} \cdot \tilde{r}_{i+1 / 2}+\mathrm{c}_{1}}{\left(\tilde{r}_{i+1 / 2}\right)^{2}+b_{0} \cdot \tilde{r}_{i+1 / 2}+\mathrm{c}_{0}}
$$

where $M$ indicates the limit value of this function when $\tilde{r}_{i+1 / 2} \rightarrow+\infty$. Waterson et al. [11] argued that a larger value of $M$ results in better resolution of steep gradients. So, the maximum value of Sweby's TVD criterion, viz. $M=2$, is selected here. The other two constraints are that Eq. (66) goes through the point $(2.0,1.75)$ with a gradient of 0.75, which guarantees a smooth switching from QUICK to the quadratic PR function at this point. Supposing that the two parameters in the denominator are equal to -1.0 (viz. $b_{0}=c_{0}=-1$ ) for simplicity and implementing the previous two constraints, one can obtain:

$$
\psi\left(\tilde{r}_{i+1 / 2}\right)=\frac{2\left(\tilde{r}_{i+1 / 2}\right)^{2}-2 \tilde{r}_{i+1 / 2}-\frac{9}{4}}{\left(\tilde{r}_{i+1 / 2}\right)^{2}-\tilde{r}_{i+1 / 2}-1} \quad \text { when } 2.0 \leq \tilde{r}_{i+1 / 2}<+\infty
$$

To sum this up, the TCDF scheme is a gradually-switching smooth multicomponent function on the positive $r$-axis and can be written in a compact form as: 


$$
\psi\left(\tilde{r}_{i+1 / 2}\right)= \begin{cases}\left(\tilde{r}_{i+1 / 2}\right)^{3}-2\left(\tilde{r}_{i+1 / 2}\right)^{2}+2 \tilde{r}_{i+1 / 2} & \text { when } 0 \leq \tilde{r}_{i+1 / 2}<0.5 \\ 0.75 \tilde{r}_{i+1 / 2}+0.25 & \text { when } 0.5 \leq \tilde{r}_{i+1 / 2}<2.0 \\ \frac{2\left(\tilde{r}_{i+1 / 2}\right)^{2}-2 \tilde{r}_{i+1 / 2}-\frac{9}{4}}{\left(\tilde{r}_{i+1 / 2}\right)^{2}-\tilde{r}_{i+1 / 2}-1} & \text { when } 2.0 \leq \tilde{r}_{i+1 / 2}<+\infty\end{cases}
$$

5.2. On the Negative $r$-axis

As is well known, conventional SS-TVD flux-limiters, based on Sweby's TVD criterion (Eq. (36)), often degenerate to the excessively diffusive FOU scheme $\left(\psi\left(\tilde{r}_{i+1 / 2}\right)=0\right)$ for $\tilde{r}_{i+1 / 2}<0$. However, as discussed in Subsection 2.2, the violation of this condition does not cause problems in practice. In the light of the striking convergence behavior of the Albada scheme (Eq. (50)) [11], its quadratic PR smooth function is used for negative $\tilde{r}_{i+1 / 2}$ values in the development of the TCDF. Therefore, the ultimate TCDF limiter can be written as:

$$
\psi\left(\tilde{r}_{i+1 / 2}\right)= \begin{cases}\tilde{r}_{i+1 / 2}\left(\tilde{r}_{i+1 / 2}+1\right) /\left(\left(\tilde{r}_{i+1 / 2}\right)^{2}+1\right) & \text { when } \tilde{r}_{i+1 / 2}<0 \\ \left(\tilde{r}_{i+1 / 2}\right)^{3}-2\left(\tilde{r}_{i+1 / 2}\right)^{2}+2 \tilde{r}_{i+1 / 2} & \text { when } 0 \leq \tilde{r}_{i+1 / 2}<0.5 \\ 0.75 \tilde{r}_{i+1 / 2}+0.25 & \text { when } 0.5 \leq \tilde{r}_{i+1 / 2}<2.0 \\ 2\left(\tilde{r}_{i+1 / 2}\right)^{2}-2 \tilde{r}_{i+1 / 2}-\frac{9}{4} & \text { when } 2.0 \leq \tilde{r}_{i+1 / 2}<+\infty \\ \left(\tilde{r}_{i+1 / 2}\right)^{2}-\tilde{r}_{i+1 / 2}-1 & \end{cases}
$$

As demonstrated in Figure 4, the TCDF limiter coincides with the third-order QUICK scheme for a wide range of $\tilde{r}_{i+1 / 2}$ values in smooth regions. Furthermore, with the aid of a cubic function and a quadratic PR function, the TCDF limiter becomes a gradually-switching smooth multi-component function on the positive $r$-axis, which contributes to achieving better convergence performance. Therefore, it is reasonable for us to believe that the newly-proposed TCDF limiter can ensure a better overall performance than the existing SS-TVD schemes in terms of accuracy and convergence.

\section{Numerical Test Cases}

This section presents several two-dimensional (2D) steady or unsteady advection test 
cases to evaluate the relative performance of nine existing classical SS-TVD flux-limiters, including Albada, Harmonic, Koren, Minmod, MUSCL, OSPRE, Superbee, UMIST and WACEB, and the newly-proposed TCDF. In these tests, the accuracy is quantitatively measured by the average $L_{2}$ norm of the difference between the exact and numerical solutions:

$$
E=\frac{1}{N} \cdot \operatorname{Sqrt}\left(\sum_{i=1}^{N}\left(q_{i}^{n}-q_{i}^{a}\right)^{2}\right)
$$

where $q_{i}^{n}$ is the calculated solution after $n$ time steps, $q_{i}^{a}$ the exact analytical solution and $N$ the number of grid points.

\subsection{Test 1: Pure convection of a step profile}

In this problem we consider the advective transport of a passive scalar by a given uniform velocity field, oblique to the horizontal direction at an angle 45 degree, in a 2D situation. The governing conservation equation of this problem is:

$$
\frac{\partial q}{\partial t}+\frac{\partial(u q)}{\partial x}+\frac{\partial(v q)}{\partial y}=0
$$

where $q$ is the advected variable, and $u=1$ and $v=1$ are the Cartesian components of the given velocity vector. As shown in Figure 5, the inlet boundary conditions are defined as:

$$
\begin{aligned}
& q(0, y)=1 \quad \text { for } \quad 0 \leq y \leq 1 \\
& q(x, 0)=0 \quad \text { for } \quad 0<x \leq 1
\end{aligned}
$$

Initially, the variable values in all the computational cells are set to 0 . The step profile provides the most stringent gradient variation, with the purpose of evaluating the method's ability to resolve a sharp front, with minimum numerical diffusion and without oscillations $[4,6]$.

A uniformly spaced mesh of $60 \times 60$ cells is employed in this test. To reach steady state, a pseudo-time stepping approach is adopted and computations are performed at six different time steps, yielding maximum Courant numbers $(\mathrm{Cu})$ of $0.4,0.8,1.2,2.0$, 2.5 and 3.0, respectively. Moreover, the first-order implicit Euler method is used for the time discretization because it allows large time steps to be taken, which is especially useful for solving steady flow problems. Specifically, the FOU value $\left(q_{i}\right)$ in Eq. (33) or Eq. (34) is implicitly discretized and the high-order correction term is explicitly treated as the source term. 
The first column in Table 1 summarizes the average errors of various SS-TVD flux-limiters along the line $x=0.7$ when the steady-state solution is achieved, and Figure 6 compares the resulting profiles of $q$ along this line for different schemes. In addition, the number of iterations needed to achieve convergence (when the residual is smaller than $10^{-6}$ ) at different Courant numbers, viz. using different relaxation factors, is listed in Table 1 for the aforementioned ten flux-limiters. The corresponding decay processes of the residuals at a Courant number of 3.0 are demonstrated in Figure 7. Note that, as shown in Figure 6, the FOU scheme does introduce unacceptable level of numerical diffusion.

Obviously, when compared with the Minmod, UMIST, Albada, Harmonic and OSPRE, the newly-proposed TCDF scheme significantly improves the accuracy of the solution. Although the former methods give better convergence properties, the latter (TCDF) is still preferred in consideration of its much better resolution and only slightly worse convergence rate, as displayed in Table 1 and Figures 6-7.

When compared to the well-known MUSCL and Koren schemes, the computational results presented in Table 1 and Figures 6-7 show that the TCDF provides a slightly more accurate solution but with a much better convergence behavior (especially at high Courant numbers), which is in accordance with the results given by the Table-B1 in Ref. [11] and demonstrates the advantage of the newly-proposed limiter once again.

As expected, in comparison with the WACEB scheme, the TCDF predicts almost the same scalar profile (actually slightly worse). However, significant improvement in convergence can be realized by using the newly-proposed limiter, which is therefore still recommended, as illustrated in Figures 6-7. Special attention needs to be paid to the well-known Superbee scheme, which is known as one of the most compressive differencing schemes, and does produce the most accurate result in this case but with the worst convergence behavior [11]. The excellent accuracy displayed by the Superbee scheme appears to be related to a property of the scheme that it tends to compress any gradient into a step profile. This is also demonstrated in Test 3 where the Superbee scheme yields severe numerical distortions for the advection of a smooth profile, which is thought to be due to its inherent squaring effect.

All in all, the numerical results clearly show that the newly-proposed TCDF scheme results in a better overall performance on accuracy and convergence in this test, compared to the remaining nine existing classical SS-TVD flux-limiters. 


\subsection{Test 2: Pure convection of a sine-square profile}

As illustrated in Figure 8, the same physical domain, governing equation, initial condition, time discretization scheme and velocity field as the step profile case are adopted in conducting the well-known advection test of a sine-square profile. The inlet boundary conditions are described as:

$$
\begin{aligned}
& q(0, y)=\sin ^{2}\left(\frac{10}{3} \pi y\right) \quad \text { for } \quad 0 \leq y \leq \frac{3}{10} \\
& q(0, y)=0 \quad \text { for } \quad 3 / 10<y \leq 1 \\
& q(x, 0)=0 \quad \text { for } 0<x \leq 1
\end{aligned}
$$

The sine-square is a relatively steep profile that enables an assessment of the influence of implementing various flux-limiters in steep gradient regions. In this test, a slightly non-uniform structured mesh of $65 \times 65$ cells is employed. Computations are performed at six different time steps as well, yielding maximum Courant numbers of $0.4,0.8,1.2,2.0,2.5$ and 3.0, respectively.

The average errors of various SS-TVD flux-limiters along the line $x=0.6$ when the steady-state is reached are listed in the first column of Table 2, and the resulting profiles of $q$ along this line for the above ten flux-limiters are presented in Figure 9. Furthermore, Table 2 gives the number of iterations needed to achieve convergence at different Courant numbers for various schemes, and the decay processes of the residuals at a Courant number of 3.0 are displayed in Figure 10.

In contrast to the Minmod, UMIST, Albada, Harmonic and OSPRE limiters, the superiority of TCDF is more clearly demonstrated than that in the first test case in view of the significant improvement in accuracy, as shown in Table 2 and Figure 9. Relative to the MUSCL scheme, TCDF has a slightly smaller average error here, which is in accordance with the result of the first test. However, in this case, TCDF leads to a slightly larger average error in comparison with the Koren limiter, which is contrary to the conclusion obtained in the previous test. Nevertheless, TCDF does show a more rapid convergence rate than both the MUSCL and Koren schemes, as evidenced in Table 2 and Figure 10. In addition, as expected, when compared to the WACEB limiter, TCDF results in a slightly larger average error but a significantly improved convergence property, as displayed in Figures 9-10.

Overall, almost the same conclusions as drawn from the first test can be reached in this test case. Again the TCDF scheme displays the excellent overall performance in terms of accuracy and convergence. 


\subsection{Test 3: Pure convection of a semi-ellipse profile}

The semi-ellipse profile, due to the combination of opposite gradient discontinuities at the base with a region of slowly varying curvature without a sharp maximum, serves as a good benchmark problem for the assessment of the steepening/clipping characteristics of various SS-TVD flux-limiters [4, 6, 19].

As described in Figure 11, the same physical domain, governing equation, initial condition, velocity vector and time discretization scheme as the sine-square profile case are employed in this test. Further, a slightly non-uniform structured mesh of $63 \times 63$ cells is utilized and computations have been carried out at six different time steps, yielding maximum Courant numbers of 0.4, 0.8, 1.2, 2.0, 2.5 and 3.0, respectively. The inlet boundary conditions are:

$$
\begin{array}{lll}
q(0, y)=\sqrt{1-[y /(1 / 6)]^{2}} & \text { for } & |y| \leq 1 / 6 \\
q(0, y)=0 & & \text { elsewhere }
\end{array}
$$

and

$$
\begin{array}{ll}
q(x, 0)=\sqrt{1-[x /(1 / 6)]^{2}} & \text { for }|x| \leq 1 / 6 \\
q(x, 0)=0 & \text { elsewhere }
\end{array}
$$

When the steady-state solution is obtained, the average errors along the line $x=0.7$ for different flux-limiters are summarized in the first column of Table 3, and a graphical representation of the resulting profiles of $q$ along this line is given by Figure 12. Table 3 shows the number of iterations needed to achieve convergence at different Courant numbers for various schemes, and the decay processes of the residuals at a Courant number of 3.0 are presented in Figure 13.

The advantages of TCDF over the MUSCL, Harmonic and Koren schemes are demonstrated more clearly in this test case than that in the previous test cases, because the TCDF gives a much better performance in terms of both accuracy and convergence. Particularly, as displayed in Table 3, when the Courant number is larger than 1.2, the number of iterations needed to achieve convergence actually increases with the increase of the adopted pseudo-time step for the Harmonic limiter, which can be attributed to the necessity of a stronger relaxation (i.e. a smaller pseudo time-step) for this limiter in order to rapidly reach the steady-state in this test.

Furthermore, it is interesting to observe that the Superbee scheme does tend to 
compress the relatively smooth semi-ellipse profile into a step-like profile due to its inherent squaring effect, as evidenced in Figure 12. In line with what was observed in the previous tests, the Superbee limiter yields a much worse convergence rate than the newly-proposed TCDF.

Except for the above differences, similar conclusions to those drawn in Test 1 and Test 2 can be derived in this test. The TCDF scheme again demonstrates excellent overall performance in terms of accuracy and convergence.

\subsection{Test 4: Pure convection of a one-double-step profile (Rotational)}

The previous three tests show an excellent overall performance of the TCDF in the uniform flow field. In order to evaluate the ability of the TCDF to handle non-uniform flows, the advection of a one-double-step profile in a rotational velocity field is simulated in Test 4. The test conditions are illustrated in Figure 14 and the double-step profile is specified as:

$$
\begin{array}{ll}
q(x, 0)=1 & \text { for }-0.8 \leq x \leq-0.6 \\
q(x, 0)=0 & \text { for }-0.6 \leq x \leq 0 \text { and }-1 \leq x \leq-0.8
\end{array}
$$

The double-step profile is transported clockwise from the inlet boundary $(x<0, y=0)$ to the outlet boundary $(x>0, y=0)$ by a rotational velocity field defined as:

$$
U=y, \quad V=-x
$$

This problem is solved on a uniformly spaced mesh of $120 \times 60$ cells at six different time steps, yielding maximum Courant numbers of 0.4, 0.8, 1.2, 2.0, 3.0 and 4.0 , respectively. The same governing equation, time discretization scheme and initial condition as that in Test 1 are selected to conduct this test. The first column in Table 4 summarizes the accuracy of different SS-TVD flux-limiters, and the accuracy is quantitatively measured by the average $L_{2}$ norm of the difference between the exact and numerical solutions at the outlet plane $(0 \leqslant x \leqslant 1.0, y=0)$. Further, a graphical representation of the resulting profiles of $q$ along this plane is given by Figure 15. The number of iterations needed to achieve convergence at different Courant numbers for various schemes is detailed in Table 4. The decay processes of the residuals at a Courant number of 4.0 are presented in Figure 16.

Table 4 and Figures 15-16 reveal that, in contrast to the well-known Minmod MUSCL and Koren schemes, the newly-developed TCDF limiter produces a more accurate prediction, and at the same time enjoys a much better convergence property 
in this test. This clearly demonstrates its advantages once again. Moreover, as evidenced in Table 4, in comparison with the UMIST, Albada, Harmonic and OSPRE, the TCDF limiter provides a much better accuracy and leads to only a slightly worse convergence. The superiority of the TCDF over other schemes is more distinctly shown in this test than that in the previous three uniform velocity-field tests.

As with the first three tests, WACEB produces a slightly more accurate numerical prediction than TCDF in this test. However, the latter (TCDF) does result in a much better convergence rate here, compared to the former. Although the Superbee limiter shows excellent ability to simulate discontinuities, it is not able to converge to the prescribed residual tolerance of $10^{-6}$ in this circumstance, as evidenced in Table 4. Besides, it should be noted that, when the Courant number is larger than 3.0, the number of iterations needed to achieve convergence actually increases with the increase of the pseudo-time step for the MUSCL and WACEB limiters, which means that a stronger relaxation (i.e. a smaller pseudo time-step) is required for the two limiters in order to quickly achieve convergence here.

In conclusion, the numerical results in this more challenging situation again demonstrate the apparent superiority of TCDF over the remaining nine classical SS-TVD flux-limiters in terms of accuracy and convergence. This is consistent with the conclusions drawn from the previous three test cases.

\subsection{Test 5: Pure convection of a two-double-step profile (Rotational)}

In order to investigate the performance of different SS-TVD flux-limiters in more demanding situations and further analyze their grid convergence properties involving a sequence of grids with different fineness, we shall present two other test examples, Test 5 and Test 6 , which correspond to the pure convection of different profile shapes in a prescribed rotational velocity field.

In the fifth test, illustrated in Figure 17, a two-double-step profile is advected from the inlet plane $(x<0, y=0)$ to the outlet plane $(x>0, y=0)$ by a rotational velocity field $(U=y, V=-x)$. The inlet boundary conditions are defined as:

$$
\begin{array}{ll}
q(x, 0)=1 & \text { for }-0.8 \leq x \leq-0.6 \text { and }-0.4 \leq x \leq-0.2 \\
q(x, 0)=0 & \text { for }-1 \leq x \leq-0.8,-0.6 \leq x \leq-0.4 \text { and }-0.2 \leq x \leq 0
\end{array}
$$

The same governing equation, initial condition and time discretization scheme as those in Test 4 are adopted as well. Computations are performed on uniformly-spaced 
grids of four different resolutions, consisting of $20 \times 40,40 \times 80,80 \times 160$ and $160 \times 320$ cells, respectively. Two different pseudo-time steps, yielding maximum Courant numbers of 2.0 and 3.0 respectively, are employed with the purpose of analyzing the accuracy and convergence of various schemes with relatively large CFL values.

In fact, with regard to each of the ten SS-TVD flux-limiters in question, almost the same average errors can be obtained for the aforementioned two time steps, as displayed in Table 5. When reaching the steady state, the corresponding profiles of $q$ at the outlet plane on the mesh composed of $40 \times 80$ cells are demonstrated in Figure 18. In addition, Table 6 shows the number of iterations needed to achieve convergence at different Courant numbers on various mesh resolutions for all the ten SS-TVD limiters. Specifically, for the mesh comprised of $80 \times 160$ elements, the decay processes of the residuals at a Courant number of 3.0 are presented in Figure 19.

As expected, except for the Superbee scheme, TCDF and WACEB, which nearly produce the same numerical accuracy, are the most accurate flux-limiters under this circumstance. However, as mentioned earlier, the Superbee limiter can't be applied to solve the smooth-solution transport problems, and, moreover, TCDF is able to provide a much better convergence rate than the Superbee and WACEB schemes, as proved by Table 6 and Figure 19. Actually, in this more challenging case, TCDF even possesses better convergence property than the Albada and Minmod limiters, which is contrary to the conclusions deduced from the previous four tests. In other words, the superiority of TCDF over the other schemes is even more apparent here.

The advantage of the newly proposed TCDF in terms of accuracy and convergence over the remaining flux-limiters is demonstrated once again in this test case. For brevity, besides Tables 5-6 and Figures 18-19, only the resulting profiles of $q$ at the outlet plane achieved on different levels of uniform structured meshes for the TCDF limiter are displayed in Figure 20.

\subsection{Test 6: Pure convection of a relatively smooth profile (Grid Convergence)}

In view of the fact that the formal order of accuracy can be misleading in the presence of step functions $[1,2,6]$, the advection of a relatively smooth profile should be taken into account in order to attain the true grid-convergence orders of different TVD flux-limiters. That is also the reason for omitting the calculation of the convergence orders in the previous tests.

As demonstrated in Figure 21, the sixth test case involves the pure advection of a 
relatively smooth multi-component profile from the inlet plane $(x<0, y=0)$ to the outlet plane $(x>0, y=0)$ by the same velocity field $(U=y, V=-x)$. The inlet boundary conditions are described as:

$$
\begin{array}{ll}
q(x, 0)=0.6 \cdot \sin ^{2}[(-x-0.25) \cdot 2 \pi] & \text { for }-0.25 \leq x \leq 0 \\
q(x, 0)=-0.6 \cdot \sin ^{2}[(-x-0.25) \cdot 2 \pi] & \text { for }-0.5 \leq x \leq-0.25 \\
q(x, 0)=-0.6 & \text { for }-0.6 \leq x \leq-0.5 \\
q(x, 0)=-1.2 \cdot \sin ^{2}[(-x-1.0) \cdot 1.25 \pi]+0.6 & \text { for }-1 \leq x \leq-0.6
\end{array}
$$

The same governing equation, initial condition, time discretization scheme and computational meshes as those in Test 5 are utilized as well. Only one pseudo-time step, yielding a maximum Courant number of 1.0, is chosen to conduct the calculations for simplicity.

Table 7 presents the average numerical errors of all the ten SS-TVD flux-limiters for various grid resolutions, and Figure 22 gives a graphic presentation of the resulting profiles of $q$ at the outlet plane for the mesh consisting of $80 \times 160$ cells. In addition, the corresponding profiles of $q$ at the outlet plane achieved on different levels of uniform structured meshes for the TCDF limiter are displayed in Figure 23.

We would like to emphasize that, although the well-known Superbee limiter results in much smaller numerical errors than the newly-proposed TCDF in the previous discontinuities or steep-gradients problems, the latter is actually much more accurate and leads to much better grid convergence than the former on fine grids for a relatively smooth profile, as in this test. This can be attributed to the inherent squaring effect of the Superbee scheme, which has a tendency to compress any gradient into a step-like profile and becomes even more severe with the increase of the mesh resolution, as shown by Fig. 22 and Table 7.

In addition, it is important to note that, in contrary to the conclusions derived from the first five test cases, TCDF even results in slightly smaller numerical errors than the WACEB limiter, in spite of the fact that the latter sticks to the third-order QUICK scheme in a larger range than the former. Furthermore, it can be seen from Table 7 that the TCDF, WACEB, Koren and MUSCL schemes nearly enjoy the same convergence order, which is approximately between 2.55 and 2.62 depending on the mesh resolution and is much larger than those of the remaining six flux-limiters. It is not difficult to explain the results in view of that all the aforementioned four schemes adhere to a very accurate linear scheme, such as QUICK, CUI and Fromm, in a large 
range, as displayed in Eqs. (41-43) and Eq. (69).

It can be concluded that the superiority of the TCDF limiter is even more pronounced for the advection of a relatively smooth profile.

\subsection{Test 7: Unsteady advection in a rotating flow field}

Obviously, the previous six tests focus on the comparison between different SS-TVD flux-limiters in the context of the steady-state solution of the advection equation. Although, in Section 2 and Section 3, we declare that the OTU-TVD and SS-TVD limiters are originally developed based on the unsteady and steady-state advection problems respectively, it has also been pointed out in Section 4.4 that, theoretical speaking, when the CFL values lie in the range of $[0,1]$, both kinds of limiters are applicable to unsteady advection problems. Therefore, in Test 7, an unsteady advection test is carried out, which is about the movement of a square patch of solute in a rotational velocity field, with the purpose of evaluating the performance of different OTU-TVD and SS-TVD limiters within the unsteady-calculation context.

Similar to the test parameters set by Liang et al. [66], the computational domain is contained inside a square, with a side of length $80 \mathrm{~m}$. The prescribed velocity field can be written as follows:

$$
U=-(y-40) \times \frac{2 \pi}{360} \quad, \quad V=(x-40) \times \frac{2 \pi}{360}
$$

Initially, unit concentration is specified within a square, which has a side length of $8 \mathrm{~m}$ and is centered at $x=20 \mathrm{~m}$ and $y=40 \mathrm{~m}$, and the concentration elsewhere is set to zero, as illustrated in Figure 24. Eq. (80) indicates that the concentration field rotates counter-clockwise around the center of the domain as a rigid body, and one rotation takes $360 \mathrm{~s}$. In this pure advection scenario, the square column should theoretically return to its original position after one rotation, with its original shape.

Computations have been conducted on two mesh resolutions (viz. $80 \times 80$ and $160 \times 160$ uniformly spaced cells) with five different time steps (i.e. $0.05 s, 0.1 s, 0.2 s$, $0.4 s$ and $0.6 s$ ). Table 8 and Table 9 give the average numerical errors of different schemes, including the aforementioned ten SS-TVD limiters and other four OTUTVD limiters, namely the CFL-MUSCL (Eq. (24)), CFL-WACEB (Eq. (22)), CFLSuperbee (Eq. (23)) and CFL-Koren (Eq. (21), also referred to as the Arora-Roe limiter). The predicted concentration distributions after one rotation for all the TVD limiters in question are demonstrated by Figures 25-38. 
In terms of overall numerical accuracy, the newly-developed TCDF is slightly inferior to the WACEB and Koren limiters, but is more accurate than the OSPRE, Albada, Harmonic, Minmod and UMIST limiters. Although TCDF leads to slightly larger numerical errors than the well-known MUSCL limiter at relatively large time steps, the former is actually more accurate than the later when adopting small time steps, such as $t=0.05 \mathrm{~s}$ and $t=0.1 \mathrm{~s}$.

The only difference between MUSCL and CFL-MUSCL (or between Superbee and CFL-Superbee, between WACEB and CFL-WACEB, between Koren and CFL-Koren) lies in the adoption of different TVD limiting criteria, because both limiters share the same basic linear schemes, as shown in Eq. (24) and Eq. (41). Table 9 indicates that the latter results in more accurate numerical predictions than the former for this problem, which is mainly due to the fact that the temporal accuracy can be guaranteed by the OTU-TVD schemes, but not by the SS-TVD limiters.

Moreover, Figure 39 and Figure 40 present the concentration variations along the lines $x=20 m$ and $y=40 m$, respectively, after one rotation for the ten considered SS-TVD limiters. Due to the variation of numerical diffusion and dispersion in different directions, the shape of the square column becomes asymmetrical as it rotates. In particular, less dissipation is experienced at the inner face of the square column than in the other directions, as reflected by the more rapid decrease to zero at this location, which can be seen from Figures 39-44.

It is important to note that, although the four OTU-TVD limiters (viz. CFLMUSCL, CFL-Superbee, CFL-WACEB and CFL-Koren) possess better overall accuracy than their SS-TVD counterparts (viz. MUSCL, Superbee, WACEB and Koren) for the unsteady advection calculation, it does not mean that better resulting profiles can be achieved in all the directions with the former. In fact, it can be concluded from Figures 41-44 that, although the accuracy in the direction where the square column travels can be significantly improved, more numerical dissipation is actually introduced at the outer face of the square column by the OTU-TVD schemes relative to their SS-TVD counterparts (seeing Figures 41(b)-44(b)).

The main purpose of this test case is to verify several viewpoints discussed in Subsection 4.4. Firstly, for the CFL values in the range of $(0,1)$, both the OTU-TVD and SS-TVD limiters can be used to solve unsteady advection problems. Secondly, the former do provide more accurate numerical solutions than the latter in this circumstance, because the former guarantee both temporal and spatial accuracy, while 
only the spatial accuracy is ensured by the latter. Thirdly, for CFL values larger than 1.0, the OTU-TVD limiters are unusable, but the SS-TVD limiters are still applicable, as evidenced by Table 9. All in all, for unsteady advection problems, the former provide better accuracy for CFL numbers smaller than 1.0, whereas the latter have a greater scope of application in terms of the CFL values.

\subsection{Test 8: Lid-driven flow in a square cavity}

The previous seven tests show the superiority of TCDF for linear advection problems concerning discontinuities, steep-gradients and relatively smooth profiles. In order to investigate the performance of different SS-TVD limiters in nonlinear flow problems, the eighth test solves the lid-driven flow problem in a square cavity, which has been widely studied numerically and has become a typical benchmark problem in Computational Fluid Dynamics (CFD) and Numerical Heat Transfer (NHT) [67-68].

The computational domain and the boundary conditions are demonstrated by Figure 45, and calculations are carried out at a prescribed Reynolds number (viz. $\mathrm{Re}=5000$ ), which defined as $R_{e}=\rho U_{0} L / \mu$, with the side length of the cavity $L=1$, the velocity at the top face $U_{0}=1$, the fluid density $\rho=1$ and the dynamic viscosity $\mu=0.0002$. The motion of the fluid is governed by the Navier-Stokes equations:

$$
\begin{gathered}
\frac{\partial \rho}{\partial t}+\frac{\partial \rho u_{i}}{\partial x_{i}}=0 \\
\frac{\partial \rho u_{i}}{\partial t}+\nabla \cdot\left(\rho u_{i} u_{j}\right)=\nabla \cdot T+\rho g_{i}
\end{gathered}
$$

where the subscripts $i$ and $j$ indicate the $i^{\text {th }}$ and $j^{\text {th }}$ components of the Cartesian coordinates respectively, $u$ is velocity, $g$ is the gravitational acceleration, $t$ represents the time and $T$ is the stress tensor which contains pressure $P$.

The Pressure Implicit with Splitting of Operators (PISO) algorithm [1, 2] is employed to deal with the pressure-velocity coupling problem on a collocated grid system in the context of finite volume method (FVM). The same technique is adopted to deal with the diffusion and source terms, but ten different SS-TVD flux-limiters are employed to discretize the advection term. Calculations are carried out on a uniformly spaced mesh of $160 \times 160$ cells, and the pseudo-time step is set to $0.1 s$ for this steady flow problem. In addition, the benchmark solution of Erturk and Corke [68] based on a fine uniform grid mesh of $601 \times 601$ cells serves as the exact solution. 
Figures 46 presents the steady-state velocity vector of the flow field when using the newly-developed TCDF limiter, and Tables 10-11 give the $U$-velocity profiles along the vertical centerline of the cavity and the $V$-velocity profiles along the horizontal centerline of the cavity, respectively. The difference between the results of various TVD limiters is relatively small because of the existing of the diffusion and source terms. For brevity and clarity, we only give a graphic representation of the velocity profiles for three representative SS-TVD limiters, namely the TCDF, Superbee and Minmod, in Figures 47-48.

It is obvious that the well-known Superbee limiter, representing the upper bound of the second-order SS-TVD region [30], does lead to the severe squaring effect when used to capture a relative smooth solution. Actually, as aforementioned, with the increase of the mesh resolution, this scheme will produce even more distorted profiles in this relatively smooth problem. Besides, the Minmod limiter does result in the excessive numerical dissipation, as this scheme follows the lower bound of the second-order SS-TVD region. Nevertheless, the newly developed TCDF limiter is able to provide a well-resolved solution, which exhibits its advantage once again over the classical Superbee and Minmod limiters.

Tables 10-11 indicate that the TCDF, WACEB, Koren and Harmonic schemes actually enjoy almost the same accuracy here. When compared with the well-known MUSCL scheme, they produce more accurate predictions near the center of the cavity, but the MUSCL scheme results in slightly better numerical results near the walls. The above five limiters generate more accurate solutions than the other five schemes, namely OSPRE, Albada, Minmod, UMIST and Superbee.

\subsection{Summary of the test results}

Overall, in this section, eight test cases are carried out in order to evaluate the numerical performance of the TCDF in terms of accuracy and convergence. Numerical results demonstrate that, when it comes to the advection of discontinuities or steep-gradients, the well-known Superbee scheme does produce the most accurate solutions, as displayed in Tests 1-2, Tests 4-5 and Test 7. However, two inherent drawbacks exist in this flux-limiter, namely the bad convergence properties in the process of obtaining the steady-state solution and the poor accuracy when used to predict a relatively smooth solution. For instance, in Tests 1-5, the Superbee scheme needs much more iteration steps to meet the convergence condition, and, in some 
situations, it cannot converge to the prescribed residual tolerance. Moreover, if utilized to solve a relatively smooth solution, this limiter will unavoidably lead to excessive numerical distortion, especially on high-resolution meshes, due to its inherent squaring effect, viz. the tendency to compress any gradient into a step-like profile, as evidenced in Test 3, Test 6 and Test 8.

For the reasons given above, it is necessary to develop a universal SS-TVD limiter, aiming for general situations (viz. the coexistence of discontinuities, steepgradients and smooth regions in the solution). Apparently, in spite of its remarkable superiority in terms of simulating the advection of discontinuities and steep-gradients, the classical Superbee limiter is not appropriate for this purpose, because it fails to resolve relatively smooth parts of the solution properly.

On the one hand, except for the Superbee scheme, TCDF is able to provide a better overall performance than the other existing SS-TVD limiters under the condition of discontinuities and steep-gradients, as evidenced in Tests 1-2, Tests 4-5 and Test 7. On the other hand, when it comes to the smooth-solution circumstance, TCDF can produce much better predictions than the Superbee limiter, and is one of the most accurate SS-TVD limiters when compared with the other existing ones, as displayed in Test 3, Test 6 and Test 8. Based on the above discussions, we believe that TCDF serves as one of the best SS-TVD limiters currently available for general situations. Additionally, it can be concluded from Test 7 that, for the CFL values lower than 1.0, the OTU-TVD limiters do provide more accurate solutions than their SS-TVD counterparts, because the former guarantee both the temporal and spatial accuracy, while only the spatial accuracy can be ensured by the latter. The above conclusions are in accordance with the theoretical analysis in Sections 2-4.

\section{CONCLUSIONS}

Firstly, most TVD schemes available in the literature are reviewed and their design principles are discussed. These TVD schemes have been grouped into three broad categories, viz. OTU-TVD, MTU-TVD and SS-TVD, based on the types of flux-limiters they use. It is shown that different flux-limiter forms are necessary for various types of discretization methods (steady or unsteady, time-space-coupled or time-space-separated) in order to achieve good accuracy, convergence and efficiency. Secondly, a refined CFL-independent SS-TVD limiter, referred to as TCDF, is proposed for steady-state computations based on the review. Essentially, TCDF 
coincides with the well-known third-order QUICK scheme in smooth regions to guarantee high accuracy, and employs a gradually-switching smooth function on the positive $r$-axis with the aid of a cubic function and a quadratic PR function to achieve good convergence. Nine existing classical SS-TVD limiters, including Albada, Harmonic, Koren, Minmod, MUSCL, OSPRE, Superbee, UMIST and WACEB, are compared with the newly-proposed TCDF by solving eight $2 \mathrm{D}$ flow problems. The numerical results consistently show that TCDF results in a better overall performance in terms of accuracy and convergence in general situations.

\section{ACKNOWLEDGEMENTS}

The authors gratefully acknowledge the financial support provided by the National Natural Science Foundation of China (Grant No. 51279082 and 51511130073) and the support from Australian Research Council through a Discovery Grant (Project ID: DP110105171). 


\section{REFERENCES}

1. O. Ubbink, R.I. Issa. A method for capturing sharp fluid interfaces on arbitrary meshes. Journal of Computational Physics; 153 (1) (1999), 26-50.

2. D. Zhang, C. Jiang, D. Liang, Z. Chen, Y. Yang, Y. Shi. A refined volume-of-fluid algorithm for capturing sharp fluid interfaces on arbitrary meshes. Journal of Computational Physics, 274 (2014), 709-736.

3. R. Scardovelli, S. Zaleski. Direct numerical simulation of free-surface and interfacial flow. Annual Review of Fluid Mechanics, 31 (1) (1999), 567-603.

4. D. Zhang, C. Jiang, L. Cheng, D. Liang. A refined $r$-factor algorithm for TVD schemes on arbitrary unstructured meshes. International Journal for Numerical Methods in Fluids, DOI: 10.1002/fld.4073 (2015).

5. B.P. Leonard, J.E. Drummond. Why you should not use 'Hybrid', 'Power-Law' or related exponential schemes for convective modelling - there are much better alternatives. International Journal for Numerical Methods in Fluids, 20 (6) (1995), 421-442.

6. D. Zhang, C. Jiang, C. Yang, Y. Yang. Assessment of different reconstruction techniques for implementing the NVSF schemes on unstructured meshes. International Journal for Numerical Methods in Fluids, 74 (3) (2014), 189-221.

7. T. Hayase, J.A.C. Humphrey, R. Greif. A consistently formulated QUICK scheme for fast and stable convergence using finite-volume iterative calculation procedures. Journal of Computational Physics, 98 (1) (1992), 108-118.

8. D.B. Spalding. A novel finite difference formulation for differential expressions involving both first and second derivatives. International Journal for Numerical Methods in Engineering, 4 (4) (1972), 551-559.

9. P. Tamamidis, D.N. Assanis. Evaluation of various high-order-accuracy schemes with and without flux limiters. International Journal for Numerical Methods in Fluids, 16 (10) (1993), 931-948.

10. V. Daru, C. Tenaud. High order one-step monotonicity-preserving schemes for unsteady compressible flow calculations. Journal of Computational Physics, 193 (2) (2004), 563-594.

11. N.P. Waterson, H. Deconinck. Design principles for bounded higher-order convection schemes - a unified approach. Journal of Computational Physics, 224 (1) (2007), 182-207.

12. R.J. LeVeque. High-resolution conservative algorithms for advection in incompressible flow. SIAM Journal on Numerical Analysis, 33 (2) (1996), 627-665. 
13. Y. Wang, K. Hutter. Comparisons of numerical methods with respect to convectively dominated problems. International Journal for Numerical Methods in Fluids, 37 (6) (2001), 721-745.

14. V. Venkatakrishnan. Convergence to steady state solutions of the Euler equations on unstructured grids with limiters. Journal of Computational Physics, 118 (1) (1995), $120-130$.

15. F. Denner, B.G. van Wachem. TVD differencing on three-dimensional unstructured meshes with monotonicity-preserving correction of mesh skewness. Journal of Computational Physics, 298 (2015), 466-479.

16. J.P. Boris, D.L. Book. Flux-corrected transport. I. SHASTA, A fluid transport algorithm that works. Journal of Computational Physics, 11 (1) (1973), 38-69.

17. S.T. Zalesak, Fully multidimensional flux-corrected transport algorithms for fluids. Journal of Computational Physics, 31 (3) (1979), 335-362.

18. G. Tóth, D. Odstrčil. Comparison of some flux corrected transport and total variation diminishing numerical schemes for hydrodynamic and magneto-hydrodynamic problems. Journal of Computational Physics, 128 (1) (1996), 82-100.

19. M.A. Alves, P.J. Oliveira, F.T. Pinho. A convergent and universally bounded interpolation scheme for the treatment of advection. International Journal for Numerical Methods in Fluids, 41 (1) (2003), 47-75.

20. H. Jasak, H.G. Weller, A.D. Gosman. High resolution NVD differencing scheme for arbitrarily unstructured meshes. International Journal for Numerical Methods in Fluids, 31 (2) (1999), 431-449.

21. M.S. Darwish, F.H. Moukalled. Normalized variable and space formulation methodology for high-resolution schemes. Numerical Heat Transfer, 26 (1) (1994), 79-96.

22. J. Zhu. A low-diffusive and oscillation-free convection scheme. Communications in Applied Numerical Methods, 7 (3) (1991), 225-232.

23. B. Song, G.R. Liu, K.Y. Lam, R.S. Amano. On a higher-order bounded discretization scheme. International Journal for Numerical Methods in Fluids, 32 (7) (2000), 881-897.

24. M.S. Darwish. A new high-resolution scheme based on the normalized variable formulation. Numerical Heat Transfer, Part B: Fundamentals, 24 (3) (1993), 353-371.

25. B.P. Leonard. The ULTIMATE conservative difference scheme applied to unsteady one-dimensional advection. Computer Methods in Applied Mechanics and Engineering, 88 (1) (1991), 17-74.

26. P.H. Gaskell, A.K.C. Lau. Curvature-compensated convective transport: SMART, A new 
boundedness-preserving transport algorithm. International Journal for Numerical Methods in Fluids, 8 (6) (1988), 617-641.

27. M. Zijlema. On the construction of a third-order accurate monotone convection scheme with application to turbulent flows in general domains. International Journal for Numerical Methods in Fluids, 22 (7) (1996), 619-641.

28. V.G. Ferreira, R.A.B. de Queiroz, G.A.B. Lima, R.G. Cuenca, C.M. Oishi, J.L.F. Azevedo, S. McKee. A bounded upwinding scheme for computing convectiondominated transport problems. Computers \& Fluids, 57 (2012), 208-224.

29. A. Harten. High resolution schemes for hyperbolic conservation laws. Journal of Computational Physics, 49 (3) (1983), 357-393.

30. P.K. Sweby. High resolution schemes using flux limiters for hyperbolic conservation laws. SIAM Journal on Numerical Analysis, 21 (5) (1984), 995-1011.

31. B. Van Leer. Towards the ultimate conservative difference scheme. IV. A new approach to numerical convection. Journal of Computational Physics, 23 (3) (1977), 276-299.

32. W. Hundsdorfer, R.A. Trompert. Method of lines and direct discretization: a comparison for linear advection. Applied Numerical Mathematics, 13 (6) (1994), 469-490.

33. F. Kemm. A comparative study of TVD-limiters-well-known limiters and an introduction of new ones. International Journal for Numerical Methods in Fluids, 67 (4) (2011), 404-440.

34. Y.N. Jeng, U.J. Payne. An adaptive TVD limiter. Journal of Computational Physics, 118 (2) (1995), 229-241.

35. B.P. Leonard. Simple high-accuracy resolution program for convective modelling of discontinuities. International Journal for Numerical Methods in Fluids, 8 (10) (1988), 1291-1318.

36. M.K. Kadalbajoo, R. Kumar. A high resolution total variation diminishing scheme for hyperbolic conservation law and related problems. Applied Mathematics and Computation, 175 (2) (2006), 1556-1573.

37. V. Przulj, B. Basara. Bounded convection schemes for unstructured grids. 15th AIAA Computational Fluid Dynamics Conference, Anaheim, CA, 2001.

38. R.K. Dubey. Flux limited schemes: Their classification and accuracy based on total variation stability regions. Applied Mathematics and Computation, 224 (2013), 325-336.

39. M. Čada, M. Torrilhon. Compact third-order limiter functions for finite volume methods. Journal of Computational Physics, 228 (11) (2009), 4118-4145.

40. R. Artebrant, H.J. Schroll. Limiter-free third order logarithmic reconstruction. SIAM 
Journal on Scientific Computing, 28 (1) (2006), 359-381.

41. A. Harten, B. Engquist, S. Osher, S.R. Chakravarthy. Uniformly high order accurate essentially non-oscillatory schemes, III. Journal of Computational Physics, 71 (2) (1987), 231-303.

42. H.Q. Yang, A.J. Przekwas. A comparative study of advanced shock-capturing schemes applied to Burgers' equation. Journal of Computational Physics, 102(1) (1992), 139-159.

43. H. Nessyahu, E. Tadmor. Non-oscillatory central differencing for hyperbolic conservation laws. Journal of Computational Physics, 87 (2) (1990), 408-463.

44. M. Arora, P.L. Roe. A well-behaved TVD limiter for high-resolution calculations of unsteady flow. Journal of Computational Physics, 132 (1) (1997), 3-11.

45. M.S. Darwish, F. Moukalled. TVD schemes for unstructured grids. International Journal of Heat and Mass Transfer, 46 (4) (2003), 599-611.

46. L.X. Li, H.S. Liao, L.J. Qi. An improved $r$-factor algorithm for TVD schemes. International Journal of Heat and Mass Transfer, 51 (3) (2008), 610-617.

47. J. Hou, F. Simons, R. Hinkelmann. A new TVD method for advection simulation on 2D unstructured grids. International Journal for Numerical Methods in Fluids, 71 (10), (2013), 1260-1281.

48. F.S. Lien, M.A. Leschziner. Upstream monotonic interpolation for scalar transport with application to complex turbulent flows. International Journal for Numerical Methods in Fluids, 19 (6) (1994), 527-548.

49. J. Liu, M. Delshad, G.A. Pope, K. Sepehrnoori. Application of higher-order flux-limited methods in compositional simulation. Transport in Porous media, 16 (1) (1994), 1-29.

50. C.M. Oldenburg, K. Pruess. Simulation of propagating fronts in geothermal reservoirs with the implicit Leonard total variation diminishing scheme. Geothermics, 29 (1) (2000), 1-25.

51. P.L. Roe. Some contributions to the modelling of discontinuous flows. Large-scale Computations in Fluid Mechanics, 22 (1985), 163-193.

52. P. Lax, B. Wendroff. Systems of conservation laws. Communications on Pure and Applied Mathematics, 13 (2) (1960), 217-237.

53. K.B. Kuan, C.A. Lin. Adaptive QUICK-based scheme to approximate convective transport. AIAA Journal, 38 (12) (2000), 2233-2237.

54. B. Van Leer. Upwind-difference methods for aerodynamic problems governed by the Euler equations. In Large-scale Computations in Fluid Mechanics, 1 (1985), 327-336.

55. W. Hundsdorfer, B. Koren, M. Van Loon, J.G. Verwer. A positive finite-difference 
advection scheme. Journal of Computational Physics, 117 (1) (1995), 35-46.

56. S.P. Spekreijse, Multi-grid solution of the steady Euler equations, Doctoral Thesis, Delft University of Technology, The Netherlands, 1986.

57. G.D. Van Albada, B. Van Leer, W.W. Roberts Jr. A comparative study of computational methods in cosmic gas dynamics. Astronomy and Astrophysics, 108 (1982), 76-84.

58. S. Gottlieb, C.W. Shu. Total variation diminishing Runge-Kutta schemes. Mathematics of Computation of the American Mathematical Society, 67 (221) (1998), 73-85.

59. C.W. Shu, T.A. Zang, G. Erlebacher, D. Whitaker, S. Osher. High-order ENO schemes applied to two-and three-dimensional compressible flow. Applied Numerical Mathematics, 9 (1) (1992), 45-71.

60. B. Koren. A robust upwind discretization method for advection, diffusion and source terms. Amsterdam: Centrum voor Wiskunde en Informatica, (1993).

61. H.C. Yee. Construction of explicit and implicit symmetric TVD schemes and their applications. Journal of Computational Physics, 68 (1) (1987), 151-179.

62. S.R. Chakravarthy, S. Osher. A new class of high accuracy TVD schemes for hyperbolic conservation laws. AIAA paper, 85 (1985), 0363.

63. B.P. Leonard. A stable and accurate convective modelling procedure based on quadratic upstream interpolation. Computer Methods in Applied Mechanics and Engineering, 19 (1) (1979), 59-98.

64. C.H. Lin, C.A. Lin. Simple high-order bounded convection scheme to model discontinuities. AIAA Journal, 35 (3) (1997), 563-565.

65. A. Varonos, G. Bergeles. Development and assessment of a variable-order non-oscillatory scheme for convection term discretization. International Journal for Numerical Methods in Fluids, 26 (1) (1998), 1-16.

66. D. Liang, X. Wang, R.A. Falconer, B.N. Bockelmann-Evans. Solving the depthintegrated solute transport equation with a TVD-MacCormack scheme. Environmental Modelling \& Software, 25 (12) (2010), 1619-1629.

67. B.E. Griffith. An accurate and efficient method for the incompressible Navier-Stokes equations using the projection method as a preconditioner. Journal of Computational Physics, 228 (20) (2009), 7565-7595.

68. E. Erturk, T.C. Corke, C. Gökçöl. Numerical solutions of 2-D steady incompressible driven cavity flow at high Reynolds numbers. International Journal for Numerical Methods in Fluids, 48 (7) (2005), 747-774. 
Table 1. Accuracy and convergence of various SS-TVD flux-limiters at different Courant numbers for Test 1

\begin{tabular}{cccccccc}
\hline \multirow{2}{*}{ Scheme } & $\begin{array}{c}\text { Accuracy } \\
(\varepsilon)\end{array}$ & \multicolumn{7}{c}{ Convergence $\left(N_{\text {its }}\right)$} \\
\cline { 2 - 8 } & $\mathrm{Cu}=0.4$ & $\mathrm{Cu}=0.8$ & $\mathrm{Cu}=1.2$ & $\mathrm{Cu}=2.0$ & $\mathrm{Cu}=2.5$ & $\mathrm{Cu}=3.0$ \\
\hline TCDF & $7.436 \mathrm{E}-03$ & 450 & 253 & 187 & 133 & 117 & 106 \\
Minmod & $9.523 \mathrm{E}-03$ & 427 & 233 & 164 & 109 & 95 & 85 \\
UMIST & $8.690 \mathrm{E}-03$ & 408 & 220 & 153 & 109 & 94 & 84 \\
Albada & $8.543 \mathrm{E}-03$ & 409 & 219 & 153 & 102 & 87 & 76 \\
Harmonic & $8.186 \mathrm{E}-03$ & 400 & 214 & 152 & 104 & 89 & 79 \\
OSPRE & $8.313 \mathrm{E}-03$ & 403 & 216 & 150 & 103 & 88 & 77 \\
MUSCL & $7.787 \mathrm{E}-03$ & 445 & 301 & 233 & 177 & 160 & 149 \\
Koren & $7.545 \mathrm{E}-03$ & 479 & 290 & 224 & 169 & 152 & 140 \\
WACEB & $7.387 \mathrm{E}-03$ & 460 & 268 & 204 & 153 & 137 & 126 \\
Superbee & $5.816 \mathrm{E}-03$ & 554 & 366 & 297 & 235 & 216 & 203 \\
\hline
\end{tabular}

$N_{\text {its }}$ : Number of iterations needed to achieve convergence: the residual is smaller than $10^{-6}$.

Table 2. Accuracy and convergence of various SS-TVD flux-limiters at different Courant numbers for Test 2

\begin{tabular}{cccccccc}
\hline \multirow{2}{*}{ Scheme } & $\begin{array}{c}\text { Accuracy } \\
(\varepsilon)\end{array}$ & $\mathrm{Cu}=0.4$ & $\mathrm{Cu}=0.8$ & $\mathrm{Cu}=1.2$ & $\mathrm{Cu}=2.0$ & $\mathrm{Cu}=2.5$ & $\mathrm{Cu}=3.0$ \\
\cline { 2 - 7 } & $2.202 \mathrm{E}-03$ & 433 & 245 & 181 & 130 & 115 & 105 \\
\hline TCDF & 419 & 224 & 158 & 105 & 88 & 77 \\
Minmod & $6.650 \mathrm{E}-03$ & 408 & 221 & 160 & 109 & 92 & 79 \\
UMIST & $4.380 \mathrm{E}-03$ & 418 & 219 & 156 & 105 & 89 & 78 \\
Albada & $4.537 \mathrm{E}-03$ & 399 & 216 & 155 & 104 & 89 & 79 \\
Harmonic & $3.309 \mathrm{E}-03$ & 438 & 230 & 161 & 104 & 89 & 82 \\
OSPRE & $3.752 \mathrm{E}-03$ & 461 & 287 & 233 & 183 & 166 & 154 \\
MUSCL & $2.247 \mathrm{E}-03$ & 483 & 291 & 224 & 171 & 155 & 145 \\
Koren & $2.037 \mathrm{E}-03$ & 453 & 267 & 209 & 156 & 139 & 128 \\
WACEB & $2.026 \mathrm{E}-03$ & 458 & 263 & 226 & 195 \\
Superbee & $5.599 \mathrm{E}-04$ & 586 & 351 & 255 & 226 & 205 & 195 \\
\hline
\end{tabular}

$N_{\text {its }}$ : Number of iterations needed to achieve convergence: the residual is smaller than $10^{-6}$. 
Table 3. Accuracy and convergence of various SS-TVD flux-limiters at different Courant numbers for Test 3

\begin{tabular}{|c|c|c|c|c|c|c|c|}
\hline \multirow{2}{*}{ Scheme } & \multirow{2}{*}{$\begin{array}{l}\text { Accuracy } \\
(\varepsilon)\end{array}$} & \multicolumn{6}{|c|}{ Convergence $\left(N_{\text {its }}\right)$} \\
\hline & & $\mathrm{Cu}=0.4$ & $\mathrm{Cu}=0.8$ & $\mathrm{Cu}=1.2$ & $\mathrm{Cu}=2.0$ & $\mathrm{Cu}=2.5$ & $\mathrm{Cu}=3.0$ \\
\hline TCDF & $2.399 \mathrm{E}-03$ & 591 & 321 & 231 & 158 & 135 & 122 \\
\hline Minmod & 4.296E-03 & 602 & 315 & 218 & 139 & 116 & 101 \\
\hline UMIST & $3.381 \mathrm{E}-03$ & 572 & 298 & 207 & 135 & 114 & 100 \\
\hline Albada & $3.139 \mathrm{E}-03$ & 599 & 312 & 216 & 139 & 116 & 101 \\
\hline Harmonic & 2.871E-03 & 585 & 304 & 211 & 262 & 290 & 320 \\
\hline OSPRE & $2.951 \mathrm{E}-03$ & 605 & 317 & 217 & 143 & 118 & 99 \\
\hline MUSCL & $2.701 \mathrm{E}-03$ & 611 & 411 & 299 & 196 & 173 & 159 \\
\hline Koren & $2.503 \mathrm{E}-03$ & 590 & 338 & 254 & 186 & 166 & 152 \\
\hline WACEB & 2.390E-03 & 589 & 321 & 233 & 170 & 149 & 135 \\
\hline Superbee & 2.297E-03 & 719 & 400 & 345 & 310 & 302 & 299 \\
\hline
\end{tabular}

$N_{\text {its }}$ : Number of iterations needed to achieve convergence: the residual is smaller than $10^{-6}$.

Table 4. Accuracy and convergence of various SS-TVD flux-limiters at different Courant numbers for Test 4

\begin{tabular}{cccccccc}
\hline \multirow{2}{*}{ Scheme } & $\begin{array}{c}\text { Accuracy } \\
(\varepsilon)\end{array}$ & \multicolumn{7}{c}{ Convergence $\left(N_{\text {its }}\right)$} \\
\cline { 2 - 7 } & $\mathrm{Cu}=0.4$ & $\mathrm{Cu}=0.8$ & $\mathrm{Cu}=1.2$ & $\mathrm{Cu}=2.0$ & $\mathrm{Cu}=3.0$ & $\mathrm{Cu}=4.0$ \\
\hline TCDF & $8.610 \mathrm{E}-03$ & 1194 & 612 & 419 & 269 & 196 & 160 \\
Minmod & $1.104 \mathrm{E}-02$ & 1578 & 748 & 492 & 304 & 215 & 173 \\
UMIST & $9.961 \mathrm{E}-03$ & 1164 & 598 & 410 & 260 & 185 & 148 \\
Albada & $9.662 \mathrm{E}-03$ & 1200 & 617 & 421 & 265 & 187 & 149 \\
Harmonic & $9.305 \mathrm{E}-03$ & 1141 & 591 & 407 & 259 & 185 & 148 \\
OSPRE & $9.422 \mathrm{E}-03$ & 1184 & 611 & 419 & 265 & 189 & 150 \\
MUSCL & $8.893 \mathrm{E}-03$ & 1395 & 885 & 579 & 383 & 281 & 354 \\
Koren & $8.662 \mathrm{E}-03$ & 1155 & 601 & 414 & 305 & 271 & 203 \\
WACEB & $8.587 \mathrm{E}-03$ & 1192 & 613 & 422 & 276 & 225 & 240 \\
Superbee & $6.530 \mathrm{E}-03$ & $/$ & $/$ & $/$ & $/$ & $/$ & $/$ \\
\hline
\end{tabular}

$N_{\text {its }}:$ Number of iterations needed to achieve convergence: the residual is smaller than $10^{-6}$. 
Table 5. Accuracy of various SS-TVD flux-limiters on uniformly-spaced grids of four different resolutions in Test 5

\begin{tabular}{cccccc}
\hline Mesh & TCDF & MUSCL & WACEB & Koren & OSPRE \\
\hline $20 \times 40$ & $5.116 \mathrm{E}-02$ & $4.773 \mathrm{E}-02$ & $4.907 \mathrm{E}-02$ & $4.789 \mathrm{E}-02$ & $6.073 \mathrm{E}-02$ \\
$40 \times 80$ & $2.229 \mathrm{E}-02$ & $2.341 \mathrm{E}-02$ & $2.214 \mathrm{E}-02$ & $2.248 \mathrm{E}-02$ & $2.652 \mathrm{E}-02$ \\
$80 \times 160$ & $1.316 \mathrm{E}-02$ & $1.391 \mathrm{E}-02$ & $1.307 \mathrm{E}-02$ & $1.327 \mathrm{E}-02$ & $1.558 \mathrm{E}-02$ \\
$160 \times 320$ & $7.600 \mathrm{E}-03$ & $8.143 \mathrm{E}-03$ & $7.553 \mathrm{E}-03$ & $7.692 \mathrm{E}-03$ & $9.061 \mathrm{E}-03$ \\
\hline
\end{tabular}

Table 5. (Continued)

\begin{tabular}{cccccc}
\hline Mesh & Albada & Harmonic & Minmod & UMIST & Superbee \\
\hline $20 \times 40$ & $6.577 \mathrm{E}-02$ & $5.719 \mathrm{E}-02$ & $7.325 \mathrm{E}-02$ & $5.980 \mathrm{E}-02$ & $2.835 \mathrm{E}-02$ \\
$40 \times 80$ & $2.861 \mathrm{E}-02$ & $2.553 \mathrm{E}-02$ & $3.537 \mathrm{E}-02$ & $2.840 \mathrm{E}-02$ & $1.320 \mathrm{E}-02$ \\
$80 \times 160$ & $1.629 \mathrm{E}-02$ & $1.518 \mathrm{E}-02$ & $1.965 \mathrm{E}-02$ & $1.700 \mathrm{E}-02$ & $7.132 \mathrm{E}-03$ \\
$160 \times 320$ & $9.470 \mathrm{E}-03$ & $8.840 \mathrm{E}-03$ & $1.165 \mathrm{E}-02$ & $1.007 \mathrm{E}-02$ & $3.782 \mathrm{E}-03$ \\
\hline
\end{tabular}

Table 6. Convergence of various SS-TVD flux-limiters on uniformly-spaced grids of four different resolutions at different Courant numbers in Test 5

\begin{tabular}{ccccccc}
\hline $\begin{array}{c}\text { CFL } \\
\text { Number }\end{array}$ & Mesh & TCDF & MUSCL & WACEB & Koren & OSPRE \\
\hline & $20 \times 40$ & 112 & 117 & 167 & 118 & 130 \\
& $40 \times 80$ & 173 & 795 & 176 & 287 & 172 \\
& $80 \times 160$ & 268 & $(3.89 \mathrm{E}-06)$ & $(4.98 \mathrm{E}-06)$ & 296 & 256 \\
& $160 \times 320$ & 453 & 895 & $(3.51 \mathrm{E}-06)$ & $(1.27 \mathrm{E}-06)$ & 435 \\
3.0 & & & & & \\
& Mesh & Albada & Harmonic & Minmod & UMIST & Superbee \\
& $20 \times 40$ & 128 & 520 & 125 & 105 & 175 \\
& $40 \times 80$ & 190 & 175 & 194 & 162 & $(5.70 \mathrm{E}-06)$ \\
& $80 \times 160$ & 282 & 248 & 346 & 252 & $(4.49 \mathrm{E}-04)$ \\
& $160 \times 320$ & 443 & 432 & 552 & 435 & $(6.38 \mathrm{E}-04)$ \\
\hline
\end{tabular}

Table 6. (Continued)

\begin{tabular}{ccccccc}
\hline $\begin{array}{c}\text { CFL } \\
\text { Number }\end{array}$ & Mesh & TCDF & MUSCL & WACEB & Koren & OSPRE \\
\hline \multirow{3}{*}{2.0} & $20 \times 40$ & 158 & 167 & 194 & 221 & 184 \\
& $40 \times 80$ & 249 & 923 & 258 & 268 & 248 \\
& $80 \times 160$ & 388 & $(2.74 \mathrm{E}-06)$ & $(3.91 \mathrm{E}-06)$ & 478 & 375 \\
& $160 \times 320$ & 664 & 1390 & $(2.25 \mathrm{E}-06)$ & 813 & 634
\end{tabular}




\begin{tabular}{cccccc} 
Mesh & Albada & Harmonic & Minmod & UMIST & Superbee \\
$20 \times 40$ & 182 & 688 & 179 & 143 & 208 \\
$40 \times 80$ & 274 & 236 & 281 & 233 & $(3.89 \mathrm{E}-04)$ \\
$80 \times 160$ & 418 & 360 & 527 & 366 & $(3.61 \mathrm{E}-04)$ \\
$160 \times 320$ & 657 & 631 & 849 & 636 & $(5.33 \mathrm{E}-04)$ \\
\hline
\end{tabular}

Blue Bracket: It can't converge to the prescribed residual tolerance (smaller than $10^{-6}$ ).

Table 7. Accuracy and convergence orders of various SS-TVD flux-limiters on grids of four different resolutions in Test 6

\begin{tabular}{ccccccccccc}
\hline Mesh & TCDF & Order & MUSCL & Order & WACEB & Order & Koren & Order & OSPRE & Order \\
\hline $20 \times 40$ & $8.779 \mathrm{E}-3$ & - & $9.812 \mathrm{E}-3$ & - & $8.930 \mathrm{E}-3$ & - & $9.203 \mathrm{E}-3$ & - & $1.077 \mathrm{E}-2$ & - \\
$40 \times 80$ & $1.433 \mathrm{E}-3$ & 2.62 & $1.668 \mathrm{E}-3$ & 2.56 & $1.449 \mathrm{E}-3$ & 2.62 & $1.514 \mathrm{E}-3$ & 2.60 & $1.942 \mathrm{E}-3$ & 2.47 \\
$80 \times 160$ & $2.381 \mathrm{E}-4$ & 2.59 & $2.741 \mathrm{E}-4$ & 2.60 & $2.390 \mathrm{E}-4$ & 2.60 & $2.491 \mathrm{E}-4$ & 2.60 & $3.464 \mathrm{E}-4$ & 2.49 \\
$160 \times 320$ & $4.067 \mathrm{E}-5$ & 2.55 & $4.640 \mathrm{E}-5$ & 2.56 & $4.072 \mathrm{E}-5$ & 2.55 & $4.235 \mathrm{E}-5$ & 2.56 & $6.340 \mathrm{E}-5$ & 2.45 \\
\hline
\end{tabular}

Table 7. (Continued)

\begin{tabular}{ccccccccccc}
\hline Mesh & Albada & Order & Harmonic & Order & Minmod & Order & UMIST & Order & Superbee & Order \\
\hline $20 \times 40$ & $1.202 \mathrm{E}-2$ & - & $1.075 \mathrm{E}-2$ & - & $1.653 \mathrm{E}-2$ & - & $1.245 \mathrm{E}-2$ & - & $8.252 \mathrm{E}-3$ & - \\
$40 \times 80$ & $2.252 \mathrm{E}-3$ & 2.42 & $1.879 \mathrm{E}-3$ & 2.52 & $3.758 \mathrm{E}-3$ & 2.14 & $2.598 \mathrm{E}-3$ & 2.26 & $2.045 \mathrm{E}-3$ & 2.01 \\
$80 \times 160$ & $4.148 \mathrm{E}-4$ & 2.44 & $3.243 \mathrm{E}-4$ & 2.53 & $9.344 \mathrm{E}-4$ & 2.01 & $5.729 \mathrm{E}-4$ & 2.18 & $7.322 \mathrm{E}-4$ & 1.48 \\
$160 \times 320$ & $7.715 \mathrm{E}-5$ & 2.43 & $5.676 \mathrm{E}-5$ & 2.51 & $2.102 \mathrm{E}-4$ & 2.15 & $1.218 \mathrm{E}-4$ & 2.23 & $1.842 \mathrm{E}-4$ & 1.99 \\
\hline
\end{tabular}


Table 8. Accuracy of various SS-TVD flux-limiters after one rotation on two mesh resolutions with five different time steps in Test 7

\begin{tabular}{ccccccc}
\hline $\begin{array}{c}\text { Time } \\
\text { Step }\end{array}$ & Mesh & TCDF & MUSCL & WACEB & Koren & OSPRE \\
\hline \multirow{2}{*}{$0.6 \mathrm{~s}$} & $80 * 80$ & $2.607 \mathrm{E}-02$ & $2.548 \mathrm{E}-02$ & $2.598 \mathrm{E}-02$ & $2.563 \mathrm{E}-02$ & $2.735 \mathrm{E}-02$ \\
& $160 * 160$ & $2.337 \mathrm{E}-02$ & $2.313 \mathrm{E}-02$ & $2.335 \mathrm{E}-02$ & $2.321 \mathrm{E}-02$ & $2.394 \mathrm{E}-02$ \\
$0.4 \mathrm{~s}$ & $80 * 80$ & $2.302 \mathrm{E}-02$ & $2.250 \mathrm{E}-02$ & $2.290 \mathrm{E}-02$ & $2.251 \mathrm{E}-02$ & $2.463 \mathrm{E}-02$ \\
& $160 * 160$ & $1.993 \mathrm{E}-02$ & $1.969 \mathrm{E}-02$ & $1.989 \mathrm{E}-02$ & $1.974 \mathrm{E}-02$ & $2.066 \mathrm{E}-02$ \\
$0.2 \mathrm{~s}$ & $80 * 80$ & $1.933 \mathrm{E}-02$ & $1.909 \mathrm{E}-02$ & $1.918 \mathrm{E}-02$ & $1.874 \mathrm{E}-02$ & $2.157 \mathrm{E}-02$ \\
& $160 * 160$ & $1.554 \mathrm{E}-02$ & $1.536 \mathrm{E}-02$ & $1.549 \mathrm{E}-02$ & $1.530 \mathrm{E}-02$ & $1.658 \mathrm{E}-02$ \\
$0.1 \mathrm{~s}$ & $80 * 80$ & $1.713 \mathrm{E}-02$ & $1.724 \mathrm{E}-02$ & $1.696 \mathrm{E}-02$ & $1.651 \mathrm{E}-02$ & $1.994 \mathrm{E}-02$ \\
& $160 * 160$ & $1.267 \mathrm{E}-02$ & $1.264 \mathrm{E}-02$ & $1.261 \mathrm{E}-02$ & $1.236 \mathrm{E}-02$ & $1.407 \mathrm{E}-02$ \\
$0.05 \mathrm{~s}$ & $80 * 80$ & $1.590 \mathrm{E}-02$ & $1.629 \mathrm{E}-02$ & $1.573 \mathrm{E}-02$ & $1.529 \mathrm{E}-02$ & $1.913 \mathrm{E}-02$ \\
& $160 * 160$ & $1.090 \mathrm{E}-02$ & $1.109 \mathrm{E}-02$ & $1.082 \mathrm{E}-02$ & $1.053 \mathrm{E}-02$ & $1.268 \mathrm{E}-02$ \\
\hline
\end{tabular}

Table 8. (Continued)

\begin{tabular}{ccccccc}
\hline $\begin{array}{c}\text { Time } \\
\text { Step }\end{array}$ & Mesh & Albada & Harmonic & Minmod & UMIST & Superbee \\
\hline \multirow{2}{*}{$0.6 \mathrm{~s}$} & $80 * 80$ & $2.817 \mathrm{E}-02$ & $2.684 \mathrm{E}-02$ & $3.194 \mathrm{E}-02$ & $2.870 \mathrm{E}-02$ & $2.068 \mathrm{E}-02$ \\
& $160 * 160$ & $2.432 \mathrm{E}-02$ & $2.372 \mathrm{E}-02$ & $2.643 \mathrm{E}-02$ & $2.483 \mathrm{E}-02$ & $2.073 \mathrm{E}-02$ \\
$0.4 \mathrm{~s}$ & $80 * 80$ & $2.559 \mathrm{E}-02$ & $2.405 \mathrm{E}-02$ & $2.984 \mathrm{E}-02$ & $2.616 \mathrm{E}-02$ & $1.730 \mathrm{E}-02$ \\
& $160 * 160$ & $2.110 \mathrm{E}-02$ & $2.040 \mathrm{E}-02$ & $2.356 \mathrm{E}-02$ & $2.169 \mathrm{E}-02$ & $1.686 \mathrm{E}-02$ \\
$0.2 \mathrm{~s}$ & $80 * 80$ & $2.272 \mathrm{E}-02$ & $2.088 \mathrm{E}-02$ & $2.755 \mathrm{E}-02$ & $2.331 \mathrm{E}-02$ & $1.335 \mathrm{E}-02$ \\
& $160 * 160$ & $1.714 \mathrm{E}-02$ & $1.626 \mathrm{E}-02$ & $2.019 \mathrm{E}-02$ & $1.786 \mathrm{E}-02$ & $1.202 \mathrm{E}-02$ \\
$0.1 \mathrm{~s}$ & $80 * 80$ & $2.121 \mathrm{E}-02$ & $1.919 \mathrm{E}-02$ & $2.633 \mathrm{E}-02$ & $2.178 \mathrm{E}-02$ & $1.089 \mathrm{E}-02$ \\
& $160 * 160$ & $1.474 \mathrm{E}-02$ & $1.368 \mathrm{E}-02$ & $1.829 \mathrm{E}-02$ & $1.557 \mathrm{E}-02$ & $8.935 \mathrm{E}-03$ \\
$0.05 \mathrm{~s}$ & $80 * 80$ & $2.046 \mathrm{E}-02$ & $1.835 \mathrm{E}-02$ & $2.572 \mathrm{E}-02$ & $2.100 \mathrm{E}-02$ & $9.418 \mathrm{E}-03$ \\
& $160 * 160$ & $1.344 \mathrm{E}-02$ & $1.224 \mathrm{E}-02$ & $1.729 \mathrm{E}-02$ & $1.431 \mathrm{E}-02$ & $6.920 \mathrm{E}-03$ \\
\hline
\end{tabular}

Table 9. Accuracy of various OTU-TVD flux-limiters and their SS-TVD counterparts after one rotation on two mesh resolutions with five different time steps in Test 7

\begin{tabular}{cccccccccc}
\hline $\begin{array}{c}\text { Time } \\
\text { Step }\end{array}$ & Mesh & MUSCL & $\begin{array}{c}\text { CFL- } \\
\text { MUSCL }\end{array}$ & WACEB & $\begin{array}{c}\text { CEL- } \\
\text { WACEB }\end{array}$ & Koren & $\begin{array}{c}\text { CFL- } \\
\text { Koren }\end{array}$ & Superbee & $\begin{array}{c}\text { CFL- } \\
\text { Superbee }\end{array}$ \\
\hline \multirow{2}{*}{$0.6 \mathrm{~s}$} & $80 * 80$ & $2.548 \mathrm{E}-02$ & $2.410 \mathrm{E}-02$ & $2.598 \mathrm{E}-02$ & $2.437 \mathrm{E}-02$ & $2.563 \mathrm{E}-02$ & $2.389 \mathrm{E}-02$ & $2.073 \mathrm{E}-02$ & $1.988 \mathrm{E}-02$ \\
& $160 * 160$ & $2.313 \mathrm{E}-02$ & $\backslash$ & $2.335 \mathrm{E}-02$ & $\backslash$ & $2.321 \mathrm{E}-02$ & $\backslash$ & $2.068 \mathrm{E}-02$ & $\backslash$ \\
$0.4 \mathrm{~s}$ & $80 * 80$ & $2.250 \mathrm{E}-02$ & $2.156 \mathrm{E}-02$ & $2.290 \mathrm{E}-02$ & $2.157 \mathrm{E}-02$ & $2.251 \mathrm{E}-02$ & $2.111 \mathrm{E}-02$ & $1.730 \mathrm{E}-02$ & $1.616 \mathrm{E}-02$ \\
& $160 * 160$ & $1.969 \mathrm{E}-02$ & $\backslash$ & $1.989 \mathrm{E}-02$ & $\backslash$ & $1.974 \mathrm{E}-02$ & $\backslash$ & $1.686 \mathrm{E}-02$ & $\backslash$ \\
$0.2 \mathrm{~s}$ & $80 * 80$ & $1.909 \mathrm{E}-02$ & $1.859 \mathrm{E}-02$ & $1.918 \mathrm{E}-02$ & $1.810 \mathrm{E}-02$ & $1.874 \mathrm{E}-02$ & $1.772 \mathrm{E}-02$ & $1.335 \mathrm{E}-02$ & $1.144 \mathrm{E}-02$ \\
& $160 * 160$ & $1.536 \mathrm{E}-02$ & $1.479 \mathrm{E}-02$ & $1.549 \mathrm{E}-02$ & $1.486 \mathrm{E}-02$ & $1.530 \mathrm{E}-02$ & $1.448 \mathrm{E}-02$ & $1.202 \mathrm{E}-02$ & $1.171 \mathrm{E}-02$ \\
$0.1 \mathrm{~s}$ & $80 * 80$ & $1.724 \mathrm{E}-02$ & $1.689 \mathrm{E}-02$ & $1.696 \mathrm{E}-02$ & $1.598 \mathrm{E}-02$ & $1.651 \mathrm{E}-02$ & $1.567 \mathrm{E}-02$ & $1.089 \mathrm{E}-02$ & $8.471 \mathrm{E}-03$ \\
& $160 * 160$ & $1.264 \mathrm{E}-02$ & $1.235 \mathrm{E}-02$ & $1.261 \mathrm{E}-02$ & $1.206 \mathrm{E}-02$ & $1.236 \mathrm{E}-02$ & $1.171 \mathrm{E}-02$ & $8.935 \mathrm{E}-03$ & $8.052 \mathrm{E}-03$ \\
$0.05 \mathrm{~s}$ & $80 * 80$ & $1.629 \mathrm{E}-02$ & $1.597 \mathrm{E}-02$ & $1.573 \mathrm{E}-02$ & $1.479 \mathrm{E}-02$ & $1.529 \mathrm{E}-02$ & $1.452 \mathrm{E}-02$ & $9.418 \mathrm{E}-03$ & $6.825 \mathrm{E}-03$ \\
& $160 * 160$ & $1.109 \mathrm{E}-02$ & $1.094 \mathrm{E}-02$ & $1.082 \mathrm{E}-02$ & $1.034 \mathrm{E}-02$ & $1.053 \mathrm{E}-02$ & $1.002 \mathrm{E}-02$ & $6.920 \mathrm{E}-03$ & $5.576 \mathrm{E}-03$ \\
\hline
\end{tabular}

'I' indicates the condition with a CFL number larger than 1.0 (viz. the OTU-TVD limiters are unusable) 
Table 10 . The $U$-velocity profiles along the vertical centerline for various SS-TVD limiters on a mesh composed $160 \times 160$ cells at a time step of $0.1 \mathrm{~s}$ in Test 8

\begin{tabular}{|c|c|c|c|c|c|c|c|c|c|c|c|}
\hline $\mathrm{Y}$ & Exact & TCDF & WACEB & Koren & Harmonic & MUSCL & Superbee & OSPRE & Albada & Minmod & UMIST \\
\hline 1.000 & 1.000 & 1.000 & 1.000 & 1.000 & 1.000 & 1.000 & 1.000 & 1.000 & 1.000 & 1.000 & 1.000 \\
\hline 0.990 & $6.87 \mathrm{E}-1$ & $6.77 \mathrm{E}-1$ & $6.77 \mathrm{E}-1$ & $6.78 \mathrm{E}-1$ & $6.77 \mathrm{E}-1$ & $6.79 \mathrm{E}-1$ & $6.83 \mathrm{E}-1$ & $6.77 \mathrm{E}-1$ & $6.76 \mathrm{E}-1$ & $6.72 \mathrm{E}-1$ & $6.75 \mathrm{E}-1$ \\
\hline 0.980 & $5.16 \mathrm{E}-1$ & $5.12 \mathrm{E}-1$ & $5.13 \mathrm{E}-1$ & $5.14 \mathrm{E}-1$ & $5.14 \mathrm{E}-1$ & $5.16 \mathrm{E}-1$ & $5.27 \mathrm{E}-1$ & $5.13 \mathrm{E}-1$ & $5.12 \mathrm{E}-1$ & $5.04 \mathrm{E}-1$ & $5.10 \mathrm{E}-1$ \\
\hline 0.970 & $4.75 \mathrm{E}-1$ & $4.68 \mathrm{E}-1$ & $4.69 \mathrm{E}-1$ & $4.70 \mathrm{E}-1$ & $4.70 \mathrm{E}-1$ & $4.73 \mathrm{E}-1$ & $4.91 \mathrm{E}-1$ & $4.68 \mathrm{E}-1$ & 4.66E-1 & $4.53 \mathrm{E}-1$ & 4.63E-1 \\
\hline 0.960 & $4.74 \mathrm{E}-1$ & 4.64E-1 & $4.65 \mathrm{E}-1$ & $4.66 \mathrm{E}-1$ & 4.65E-1 & 4.69E-1 & 4.91E-1 & 4.63E-1 & 4.61E-1 & $4.46 \mathrm{E}-1$ & 4.57E-1 \\
\hline 0.950 & 4.74E-1 & 4.64E-1 & 4.65E-1 & $4.66 \mathrm{E}-1$ & 4.64E-1 & $4.69 \mathrm{E}-1$ & $4.90 \mathrm{E}-1$ & $4.62 \mathrm{E}-1$ & 4.60E-1 & 4.45E-1 & 4.57E-1 \\
\hline 0.940 & $4.68 \mathrm{E}-1$ & $4.59 \mathrm{E}-1$ & $4.60 \mathrm{E}-1$ & $4.61 \mathrm{E}-1$ & 4.60E-1 & 4.64E-1 & 4.83E-1 & $4.57 \mathrm{E}-1$ & 4.56E-1 & $4.42 \mathrm{E}-1$ & 4.53E-1 \\
\hline 0.930 & $4.58 \mathrm{E}-1$ & $4.49 \mathrm{E}-1$ & $4.50 \mathrm{E}-1$ & $4.51 \mathrm{E}-1$ & $4.51 \mathrm{E}-1$ & $4.54 \mathrm{E}-1$ & $4.71 \mathrm{E}-1$ & $4.48 \mathrm{E}-1$ & 4.47E-1 & $4.34 \mathrm{E}-1$ & 4.44E-1 \\
\hline 0.920 & 4.45E-1 & $4.37 \mathrm{E}-1$ & $4.38 \mathrm{E}-1$ & $4.39 \mathrm{E}-1$ & $4.38 \mathrm{E}-1$ & $4.41 \mathrm{E}-1$ & 4.56E-1 & $4.36 \mathrm{E}-1$ & $4.35 \mathrm{E}-1$ & $4.24 \mathrm{E}-1$ & 4.33E-1 \\
\hline 0.910 & 4.31E-1 & $4.23 \mathrm{E}-1$ & 4.24E-1 & $4.25 \mathrm{E}-1$ & 4.24E-1 & 4.27E-1 & 4.41E-1 & $4.22 \mathrm{E}-1$ & $4.21 \mathrm{E}-1$ & 4.12E-1 & 4.19E-1 \\
\hline 0.900 & 4.16E-1 & $4.08 \mathrm{E}-1$ & $4.09 \mathrm{E}-1$ & $4.10 \mathrm{E}-1$ & $4.09 \mathrm{E}-1$ & 4.12E-1 & $4.25 \mathrm{E}-1$ & 4.07E-1 & $4.06 \mathrm{E}-1$ & $3.98 \mathrm{E}-1$ & 4.05E-1 \\
\hline 0.500 & $-3.19 \mathrm{E}-2$ & $-3.22 \mathrm{E}-2$ & $-3.23 \mathrm{E}-02$ & $-3.22 \mathrm{E}-02$ & $-3.22 \mathrm{E}-2$ & $-3.26 \mathrm{E}-2$ & $-3.46 \mathrm{E}-2$ & $-3.20 \mathrm{E}-2$ & $-3.19 E-2$ & $-3.07 E-2$ & $-3.16 \mathrm{E}-2$ \\
\hline 0.200 & $-3.10 \mathrm{E}-1$ & $-3.05 \mathrm{E}-1$ & $-3.06 \mathrm{E}-1$ & $-3.06 \mathrm{E}-1$ & $-3.06 \mathrm{E}-1$ & $-3.08 \mathrm{E}-1$ & $-3.19 \mathrm{E}-1$ & $-3.04 \mathrm{E}-1$ & $-3.04 \mathrm{E}-1$ & $-2.96 \mathrm{E}-1$ & $-3.02 \mathrm{E}-1$ \\
\hline 0.180 & $-3.29 \mathrm{E}-1$ & $-3.23 \mathrm{E}-1$ & $-3.24 \mathrm{E}-1$ & $-3.24 \mathrm{E}-1$ & $-3.24 \mathrm{E}-1$ & $-3.27 \mathrm{E}-1$ & $-3.38 \mathrm{E}-1$ & $-3.22 \mathrm{E}-1$ & $-3.21 \mathrm{E}-1$ & $-3.14 \mathrm{E}-1$ & $-3.20 \mathrm{E}-1$ \\
\hline 0.160 & $-3.47 \mathrm{E}-1$ & $-3.41 \mathrm{E}-1$ & $-3.42 \mathrm{E}-1$ & $-3.42 \mathrm{E}-1$ & $-3.42 \mathrm{E}-1$ & $-3.44 \mathrm{E}-1$ & $-3.56 \mathrm{E}-1$ & $-3.40 \mathrm{E}-1$ & $-3.39 \mathrm{E}-1$ & $-3.31 \mathrm{E}-1$ & $-3.38 \mathrm{E}-1$ \\
\hline 0.140 & $-3.65 \mathrm{E}-1$ & $-3.59 \mathrm{E}-1$ & $-3.60 \mathrm{E}-1$ & $-3.60 \mathrm{E}-1$ & $-3.60 \mathrm{E}-1$ & $-3.63 \mathrm{E}-1$ & $-3.74 \mathrm{E}-1$ & $-3.59 \mathrm{E}-1$ & $-3.58 \mathrm{E}-1$ & $-3.51 \mathrm{E}-1$ & $-3.56 \mathrm{E}-1$ \\
\hline 0.120 & $-3.88 \mathrm{E}-1$ & $-3.82 \mathrm{E}-1$ & $-3.83 \mathrm{E}-1$ & $-3.84 \mathrm{E}-1$ & $-3.83 \mathrm{E}-1$ & $-3.86 \mathrm{E}-1$ & $-3.98 \mathrm{E}-1$ & $-3.82 \mathrm{E}-1$ & $-3.81 \mathrm{E}-1$ & $-3.74 \mathrm{E}-1$ & $-3.80 \mathrm{E}-1$ \\
\hline 0.100 & $-4.17 \mathrm{E}-1$ & $-4.11 \mathrm{E}-1$ & $-4.12 \mathrm{E}-1$ & $-4.13 \mathrm{E}-1$ & $-4.12 \mathrm{E}-1$ & $-4.15 \mathrm{E}-1$ & $-4.29 \mathrm{E}-1$ & $-4.10 \mathrm{E}-1$ & $-4.09 \mathrm{E}-1$ & $-4.00 \mathrm{E}-1$ & $-4.07 \mathrm{E}-1$ \\
\hline 0.080 & $-4.42 \mathrm{E}-1$ & $-4.30 \mathrm{E}-1$ & $-4.31 \mathrm{E}-1$ & $-4.32 \mathrm{E}-1$ & $-4.30 \mathrm{E}-1$ & $-4.35 \mathrm{E}-1$ & $-4.54 \mathrm{E}-1$ & $-4.27 \mathrm{E}-1$ & $-4.25 \mathrm{E}-1$ & $-4.12 \mathrm{E}-1$ & $-4.23 \mathrm{E}-1$ \\
\hline 0.060 & $-4.27 \mathrm{E}-1$ & $-4.12 \mathrm{E}-1$ & $-4.12 \mathrm{E}-1$ & $-4.13 \mathrm{E}-1$ & $-4.11 \mathrm{E}-1$ & $-4.14 \mathrm{E}-1$ & $-4.27 \mathrm{E}-1$ & $-4.09 \mathrm{E}-1$ & $-4.07 \mathrm{E}-1$ & $-3.95 \mathrm{E}-1$ & $-4.05 \mathrm{E}-1$ \\
\hline 0.040 & $-3.48 \mathrm{E}-1$ & $-3.31 \mathrm{E}-1$ & $-3.31 \mathrm{E}-1$ & $-3.32 \mathrm{E}-1$ & $-3.33 \mathrm{E}-1$ & $-3.31 \mathrm{E}-1$ & $-3.23 \mathrm{E}-1$ & $-3.34 \mathrm{E}-1$ & $-3.34 \mathrm{E}-1$ & $-3.34 \mathrm{E}-1$ & $-3.34 \mathrm{E}-1$ \\
\hline 0.020 & $-2.22 \mathrm{E}-1$ & $-2.10 \mathrm{E}-1$ & $-2.10 \mathrm{E}-1$ & $-2.09 \mathrm{E}-1$ & $-2.11 \mathrm{E}-1$ & $-2.07 \mathrm{E}-1$ & $-1.93 \mathrm{E}-1$ & $-2.12 \mathrm{E}-1$ & $-2.14 \mathrm{E}-1$ & $-2.22 \mathrm{E}-1$ & $-2.16 \mathrm{E}-1$ \\
\hline 0.000 & 0.000 & 0.000 & 0.000 & 0.000 & 0.000 & 0.000 & 0.000 & 0.000 & 0.000 & 0.000 & 0.000 \\
\hline
\end{tabular}


Table 11 . The $V$-velocity profiles along the horizontal centerline for various SS-TVD limiters on a mesh composed $160 \times 160$ cells at a time step of $0.1 \mathrm{~s}$ in Test 8

\begin{tabular}{|c|c|c|c|c|c|c|c|c|c|c|c|}
\hline $\mathrm{X}$ & Exact & TCDF & WACEB & Koren & Harmonic & MUSCL & Superbee & OSPRE & Albada & Minmod & UMIST \\
\hline 0.000 & 0.000 & 0.000 & 0.000 & 0.000 & 0.000 & 0.000 & 0.000 & 0.000 & 0.000 & 0.000 & 0.000 \\
\hline 0.985 & $-2.44 \mathrm{E}-01$ & $-2.18 \mathrm{E}-01$ & $-2.19 \mathrm{E}-01$ & $-2.17 \mathrm{E}-01$ & $-2.15 \mathrm{E}-01$ & $-2.15 \mathrm{E}-01$ & $-2.07 \mathrm{E}-01$ & $-2.16 \mathrm{E}-01$ & $-2.16 \mathrm{E}-01$ & $-2.22 \mathrm{E}-01$ & $-2.18 \mathrm{E}-01$ \\
\hline 0.970 & $-5.02 \mathrm{E}-01$ & $-4.72 \mathrm{E}-01$ & $-4.72 \mathrm{E}-01$ & $-4.71 \mathrm{E}-01$ & $-4.70 \mathrm{E}-01$ & $-4.70 \mathrm{E}-01$ & $-4.71 \mathrm{E}-01$ & $-4.70 \mathrm{E}-01$ & $-4.70 \mathrm{E}-01$ & $-4.68 \mathrm{E}-01$ & $-4.69 \mathrm{E}-01$ \\
\hline 0.955 & $-5.70 \mathrm{E}-01$ & $-5.50 \mathrm{E}-01$ & $-5.52 \mathrm{E}-01$ & $-5.53 \mathrm{E}-01$ & $-5.51 \mathrm{E}-01$ & $-5.56 \mathrm{E}-01$ & $-5.69 \mathrm{E}-01$ & $-5.48 \mathrm{E}-01$ & $-5.46 \mathrm{E}-01$ & $-5.38 \mathrm{E}-01$ & $-5.47 \mathrm{E}-01$ \\
\hline 0.940 & $-5.14 \mathrm{E}-01$ & $-5.10 \mathrm{E}-01$ & $-5.11 \mathrm{E}-01$ & $-5.12 \mathrm{E}-01$ & $-5.13 \mathrm{E}-01$ & $-5.15 \mathrm{E}-01$ & $-5.27 \mathrm{E}-01$ & $-5.11 \mathrm{E}-01$ & $-5.11 \mathrm{E}-01$ & $-5.02 \mathrm{E}-01$ & $-5.09 \mathrm{E}-01$ \\
\hline 0.925 & $-4.60 \mathrm{E}-01$ & $-4.56 \mathrm{E}-01$ & $-4.56 \mathrm{E}-01$ & $-4.57 \mathrm{E}-01$ & $-4.57 \mathrm{E}-01$ & $-4.59 \mathrm{E}-01$ & $-4.73 \mathrm{E}-01$ & $-4.56 \mathrm{E}-01$ & $-4.55 \mathrm{E}-01$ & $-4.46 \mathrm{E}-01$ & $-4.52 \mathrm{E}-01$ \\
\hline 0.910 & $-4.32 \mathrm{E}-01$ & $-4.25 \mathrm{E}-01$ & $-4.26 \mathrm{E}-01$ & $-4.27 \mathrm{E}-01$ & $-4.26 \mathrm{E}-01$ & $-4.29 \mathrm{E}-01$ & $-4.42 \mathrm{E}-01$ & $-4.24 \mathrm{E}-01$ & $-4.23 \mathrm{E}-01$ & $-4.14 \mathrm{E}-01$ & $-4.21 \mathrm{E}-01$ \\
\hline 0.895 & $-4.15 \mathrm{E}-01$ & $-4.07 \mathrm{E}-01$ & $-4.08 \mathrm{E}-01$ & $-4.09 \mathrm{E}-01$ & $-4.09 \mathrm{E}-01$ & $-4.11 E-01$ & $-4.25 \mathrm{E}-01$ & $-4.07 \mathrm{E}-01$ & $-4.06 \mathrm{E}-01$ & $-3.96 \mathrm{E}-01$ & $-4.04 \mathrm{E}-01$ \\
\hline 0.880 & $-3.98 \mathrm{E}-01$ & $-3.91 \mathrm{E}-01$ & $-3.92 \mathrm{E}-01$ & $-3.93 E-01$ & $-3.93 \mathrm{E}-01$ & $-3.95 \mathrm{E}-01$ & $-4.08 \mathrm{E}-01$ & $-3.91 \mathrm{E}-01$ & $-3.90 \mathrm{E}-01$ & $-3.81 \mathrm{E}-01$ & $-3.88 \mathrm{E}-01$ \\
\hline 0.865 & $-3.81 \mathrm{E}-01$ & $-3.75 E-01$ & $-3.75 E-01$ & $-3.76 \mathrm{E}-01$ & $-3.76 \mathrm{E}-01$ & $-3.78 \mathrm{E}-01$ & $-3.90 \mathrm{E}-01$ & $-3.74 \mathrm{E}-01$ & $-3.73 E-01$ & $-3.65 \mathrm{E}-01$ & $-3.71 \mathrm{E}-01$ \\
\hline 0.850 & $-3.62 \mathrm{E}-01$ & $-3.57 \mathrm{E}-01$ & $-3.57 \mathrm{E}-01$ & $-3.58 \mathrm{E}-01$ & $-3.58 \mathrm{E}-01$ & $-3.60 \mathrm{E}-01$ & $-3.71 \mathrm{E}-01$ & $-3.56 \mathrm{E}-01$ & $-3.55 \mathrm{E}-01$ & $-3.48 \mathrm{E}-01$ & $-3.54 \mathrm{E}-01$ \\
\hline 0.500 & 1.17E-02 & 1.06E-02 & 1.06E-02 & 1.05E-02 & 1.05E-02 & 1.03E-02 & 9.23E-03 & $1.06 \mathrm{E}-02$ & 1.07E-02 & 1.18E-02 & 1.10E-02 \\
\hline 0.150 & $3.70 \mathrm{E}-01$ & $3.64 \mathrm{E}-01$ & $3.64 \mathrm{E}-01$ & $3.65 \mathrm{E}-01$ & $3.65 \mathrm{E}-01$ & $3.67 \mathrm{E}-01$ & $3.76 \mathrm{E}-01$ & $3.63 \mathrm{E}-01$ & $3.63 \mathrm{E}-01$ & $3.57 \mathrm{E}-01$ & $3.62 \mathrm{E}-01$ \\
\hline 0.135 & $3.88 \mathrm{E}-01$ & $3.82 \mathrm{E}-01$ & $3.82 \mathrm{E}-01$ & $3.83 \mathrm{E}-01$ & 3.83E-01 & $3.85 \mathrm{E}-01$ & $3.94 \mathrm{E}-01$ & $3.81 \mathrm{E}-01$ & $3.81 \mathrm{E}-01$ & $3.74 \mathrm{E}-01$ & $3.80 \mathrm{E}-01$ \\
\hline 0.120 & 4.07E-01 & $4.01 \mathrm{E}-01$ & $4.01 \mathrm{E}-01$ & $4.02 \mathrm{E}-01$ & $4.02 \mathrm{E}-01$ & $4.04 \mathrm{E}-01$ & $4.15 \mathrm{E}-01$ & $4.00 \mathrm{E}-01$ & 3.99E-01 & $3.92 \mathrm{E}-01$ & $3.98 \mathrm{E}-01$ \\
\hline 0.105 & $4.26 \mathrm{E}-01$ & 4.19E-01 & $4.20 \mathrm{E}-01$ & $4.21 \mathrm{E}-01$ & 4.20E-01 & $4.23 \mathrm{E}-01$ & $4.36 \mathrm{E}-01$ & 4.18E-01 & 4.17E-01 & $4.07 \mathrm{E}-01$ & $4.15 \mathrm{E}-01$ \\
\hline 0.090 & $4.40 \mathrm{E}-01$ & 4.32E-01 & 4.33E-01 & 4.34E-01 & 4.33E-01 & 4.36E-01 & $4.54 \mathrm{E}-01$ & 4.30E-01 & 4.29E-01 & 4.15E-01 & $4.26 \mathrm{E}-01$ \\
\hline 0.075 & 4.43E-01 & 4.33E-01 & 4.34E-01 & $4.35 \mathrm{E}-01$ & 4.33E-01 & $4.37 \mathrm{E}-01$ & $4.59 \mathrm{E}-01$ & $4.30 \mathrm{E}-01$ & $4.28 \mathrm{E}-01$ & $4.12 \mathrm{E}-01$ & $4.25 \mathrm{E}-01$ \\
\hline 0.060 & $4.26 \mathrm{E}-01$ & $4.16 \mathrm{E}-01$ & 4.17E-01 & $4.18 \mathrm{E}-01$ & 4.17E-01 & $4.21 \mathrm{E}-01$ & $4.41 \mathrm{E}-01$ & 4.14E-01 & $4.12 \mathrm{E}-01$ & $3.96 \mathrm{E}-01$ & $4.09 \mathrm{E}-01$ \\
\hline 0.045 & $3.87 \mathrm{E}-01$ & $3.78 \mathrm{E}-01$ & $3.78 \mathrm{E}-01$ & $3.80 \mathrm{E}-01$ & $3.79 \mathrm{E}-01$ & $3.82 \mathrm{E}-01$ & $3.96 \mathrm{E}-01$ & $3.77 \mathrm{E}-01$ & $3.75 \mathrm{E}-01$ & $3.62 \mathrm{E}-01$ & $3.72 \mathrm{E}-01$ \\
\hline 0.030 & $3.26 \mathrm{E}-01$ & $3.18 \mathrm{E}-01$ & $3.19 \mathrm{E}-01$ & $3.20 \mathrm{E}-01$ & $3.20 \mathrm{E}-01$ & $3.22 \mathrm{E}-01$ & $3.33 \mathrm{E}-01$ & $3.18 \mathrm{E}-01$ & $3.17 \mathrm{E}-01$ & $3.07 \mathrm{E}-01$ & $3.15 \mathrm{E}-01$ \\
\hline 0.015 & $2.16 \mathrm{E}-01$ & $2.11 \mathrm{E}-01$ & $2.11 \mathrm{E}-01$ & $2.12 \mathrm{E}-01$ & $2.12 \mathrm{E}-01$ & $2.13 \mathrm{E}-01$ & $2.20 \mathrm{E}-01$ & $2.10 \mathrm{E}-01$ & $2.10 \mathrm{E}-01$ & $2.03 \mathrm{E}-01$ & $2.08 \mathrm{E}-01$ \\
\hline 0.000 & 0.000 & 0.000 & 0.000 & 0.000 & 0.000 & 0.000 & 0.000 & 0.000 & 0.000 & 0.000 & 0.000 \\
\hline
\end{tabular}




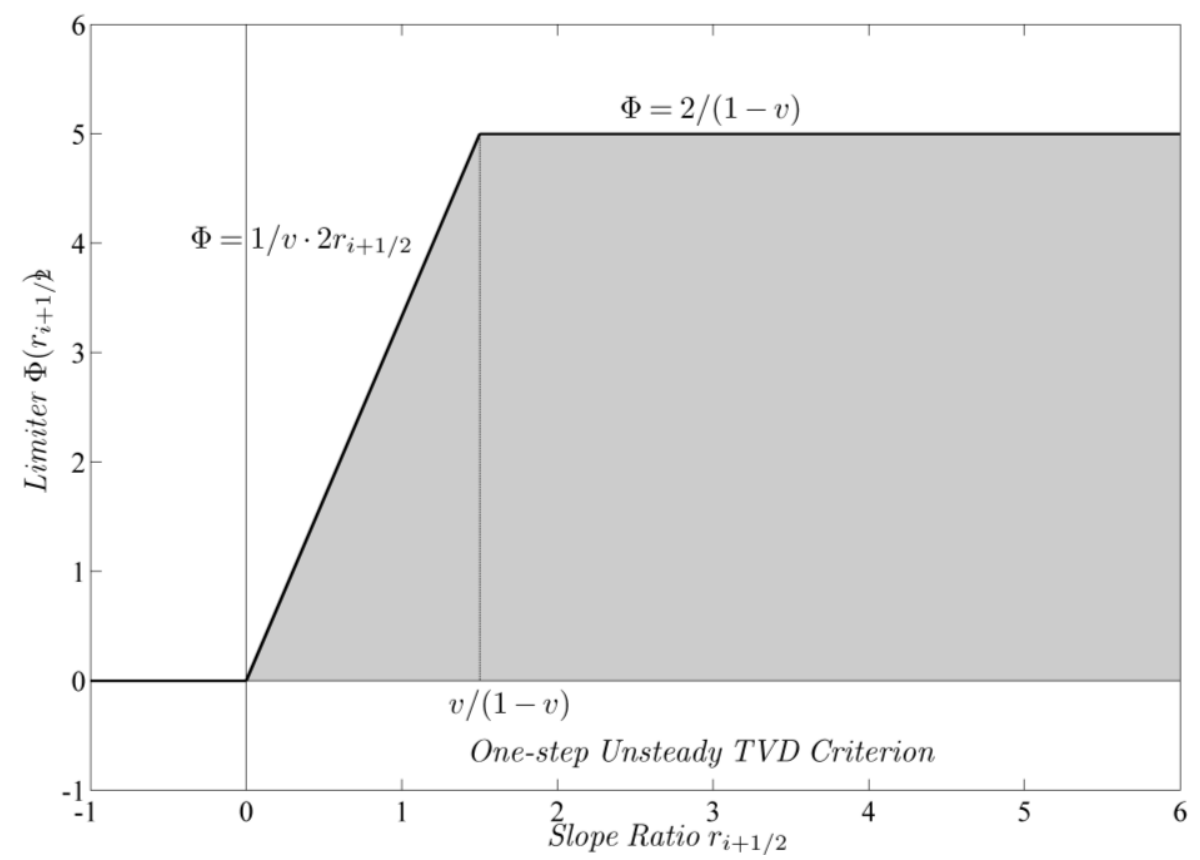

Figure 1. A graphical representation of the One-step Time-space-coupled Unsteady TVD criterion (OTU-TVD).

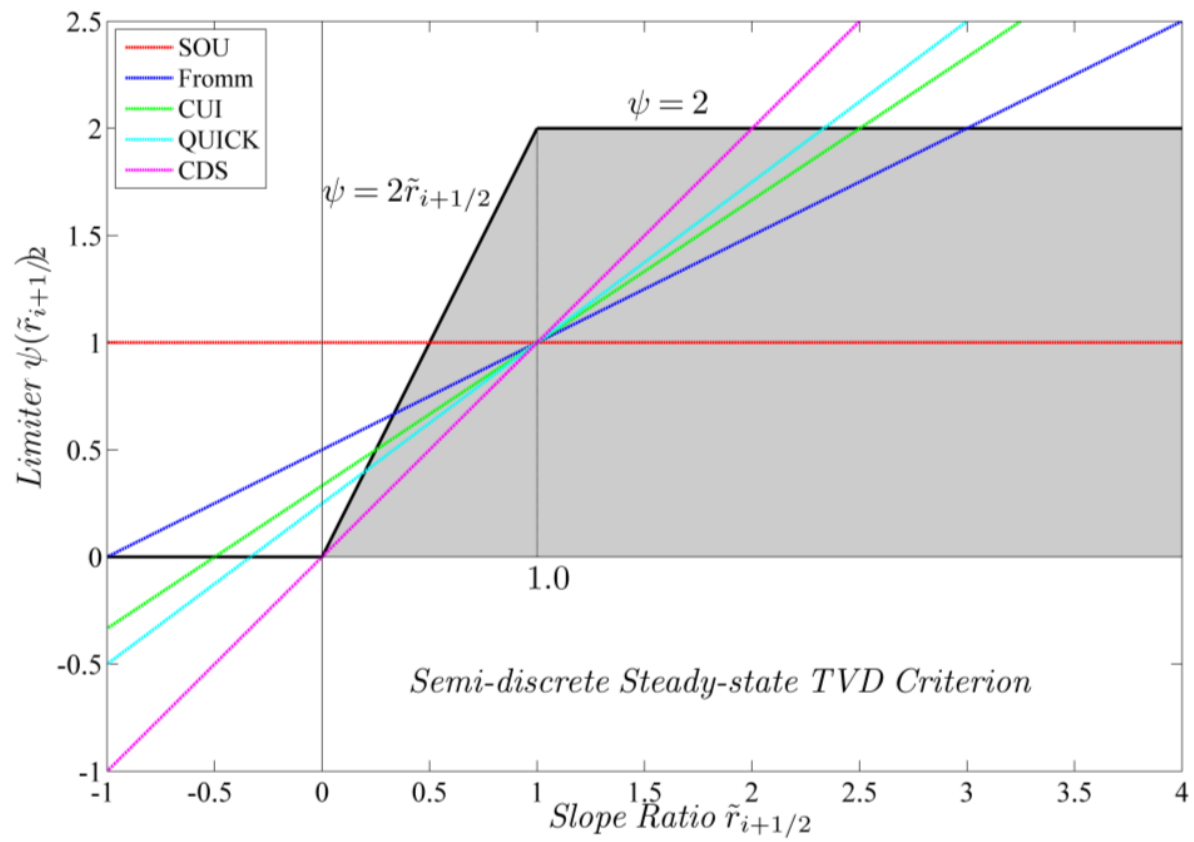

Figure 2. A graphical representation of the Semi-discrete Steady-state TVD criterion (viz. Sweby's TVD criterion) and the $k$-schemes. 


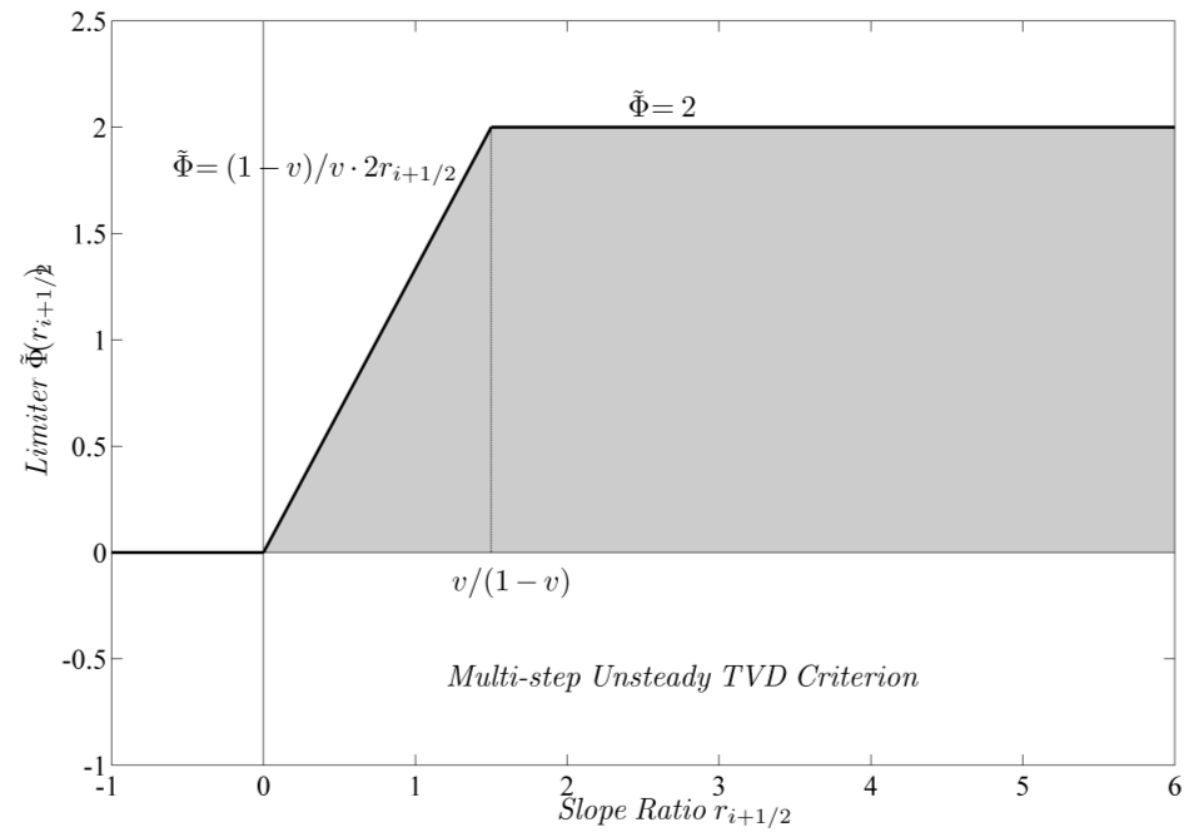

Figure 3. A graphical representation of the Multi-step Time-space-separated Unsteady TVD criterion (MTU-TVD).

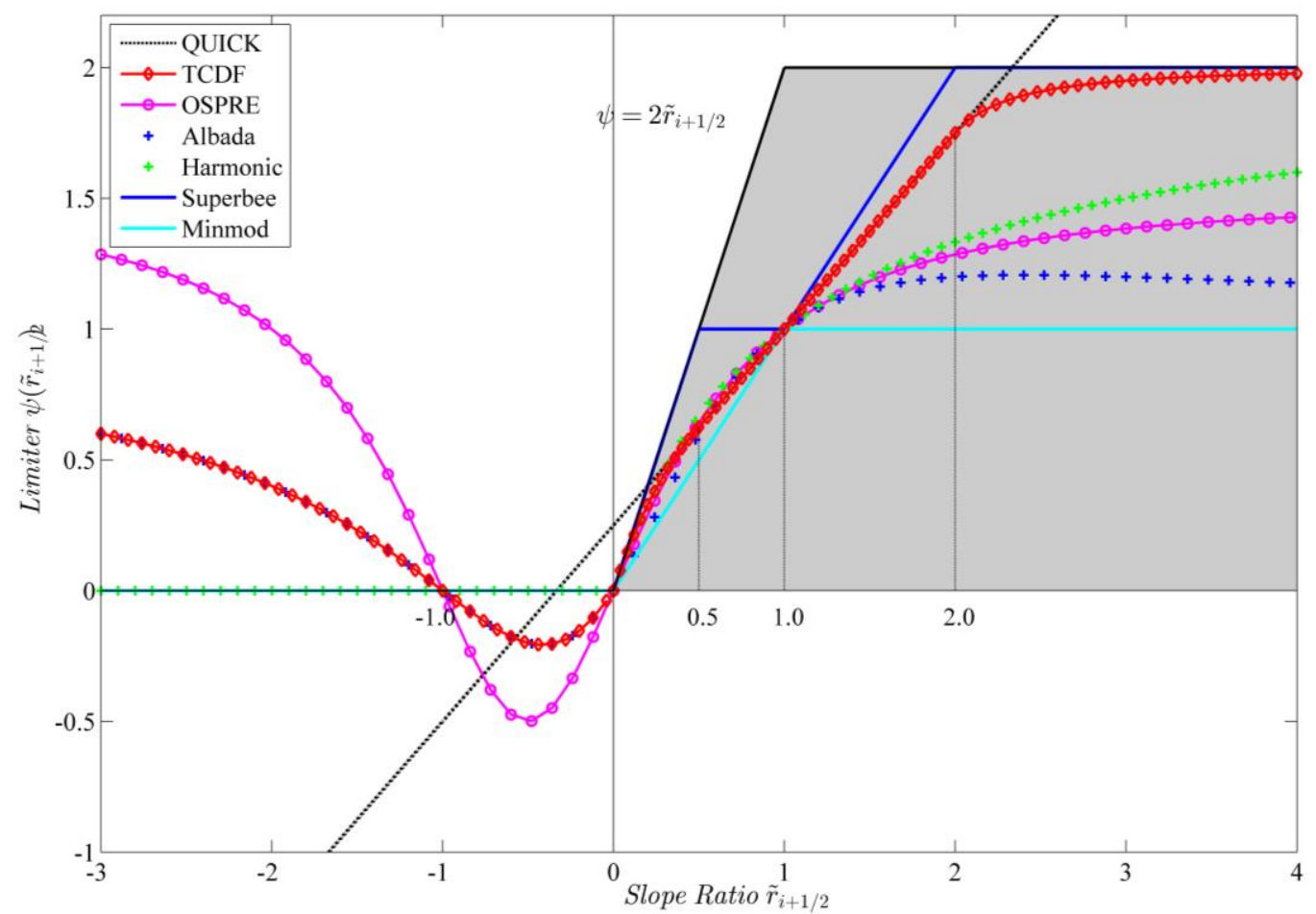

Figure 4. A graphical representation of the newly proposed TCDF limiter and several existing classical SS-TVD limiters. 


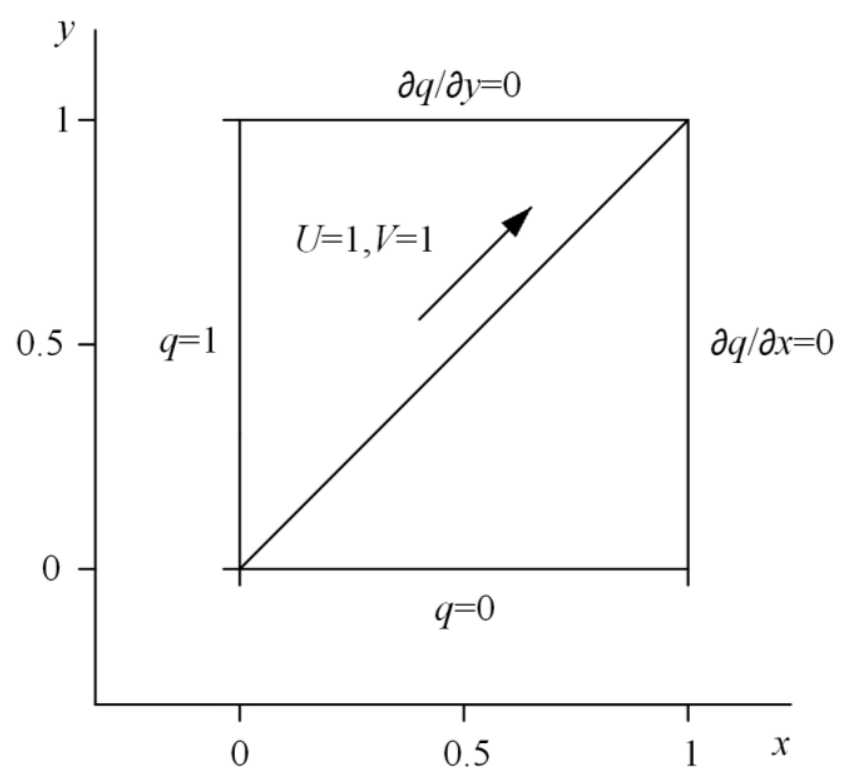

Figure 5. Pure convection of a step profile by a uniform velocity field.

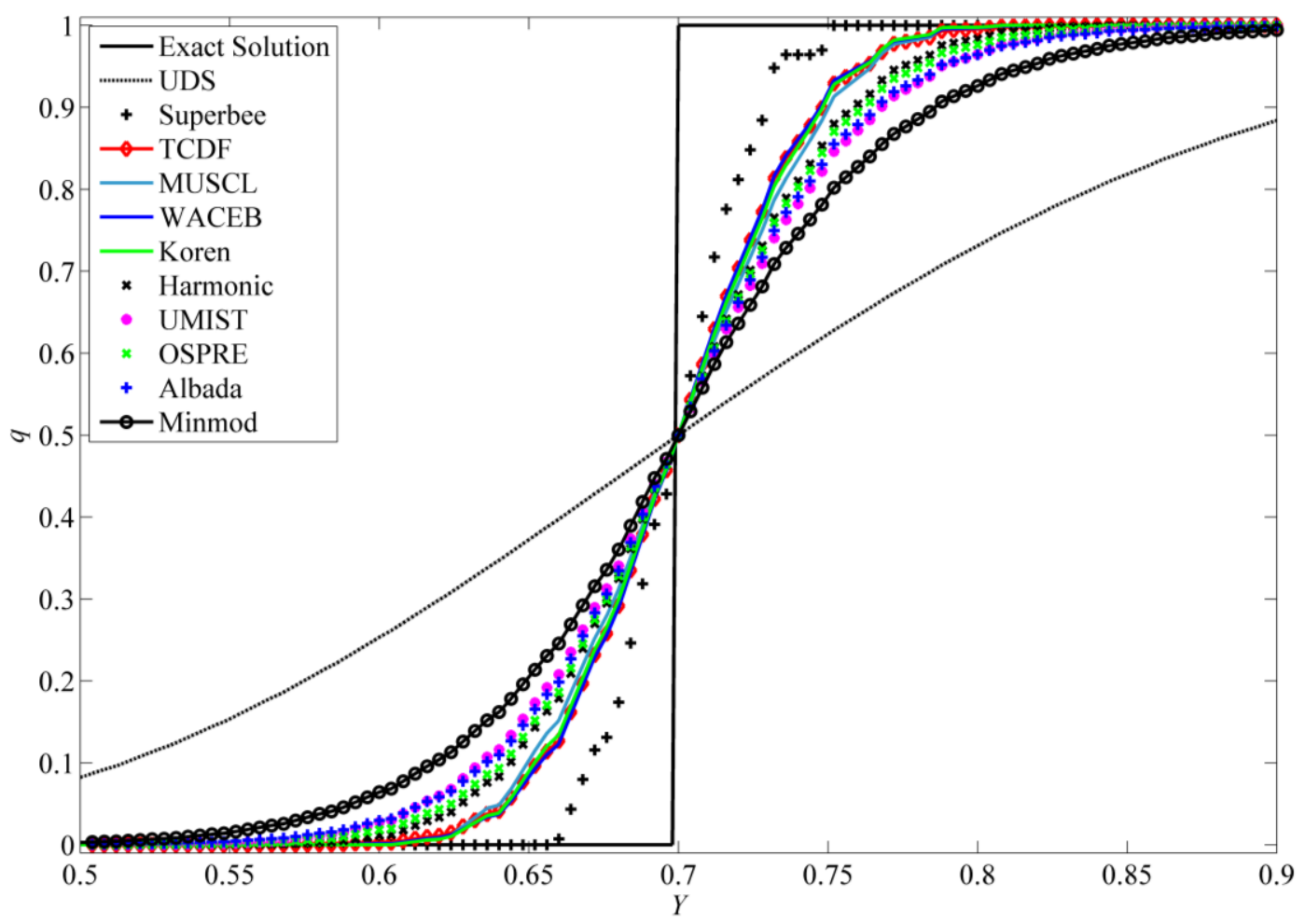

Figure 6. Comparison of accuracy of various SS-TVD flux-limiters at a Courant number of 3.0 in Test 1. 


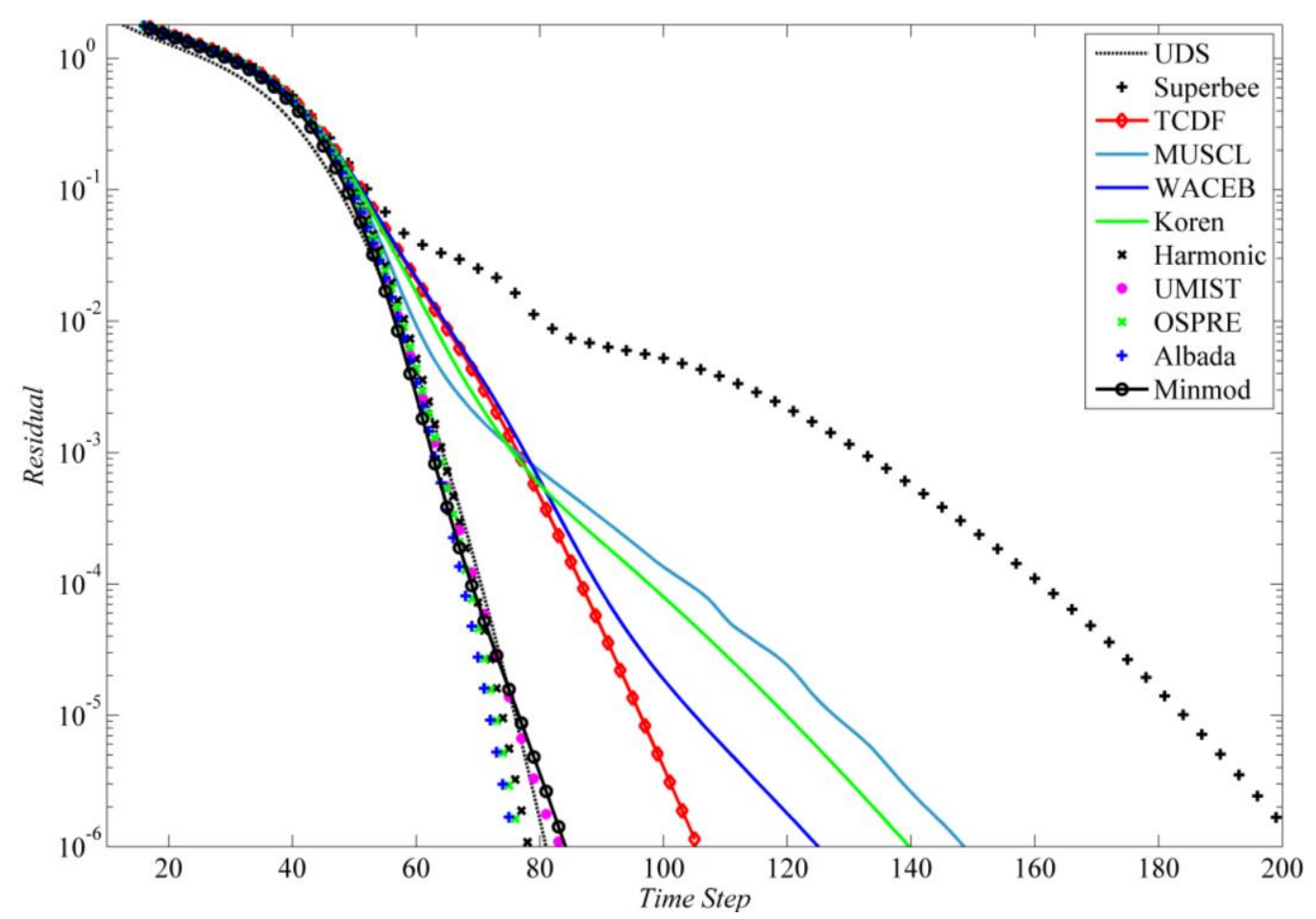

Figure 7. Comparison of convergence of various SS-TVD flux-limiters at a Courant number of 3.0 in Test 1.

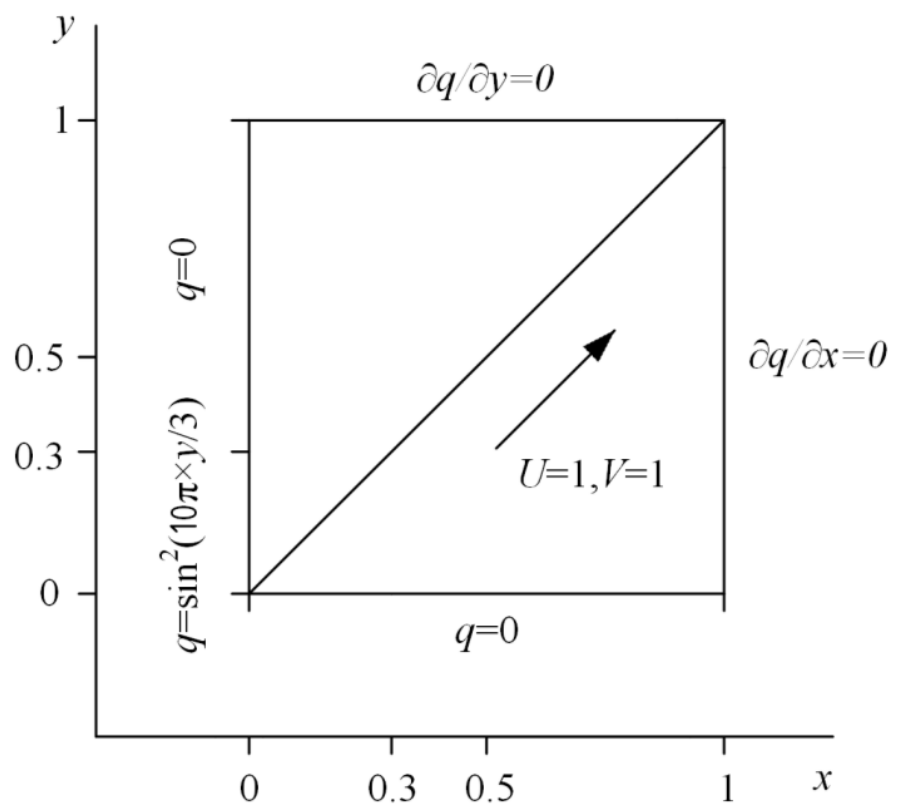

Figure 8 . Pure convection of a sine-square profile by a uniform velocity field. 


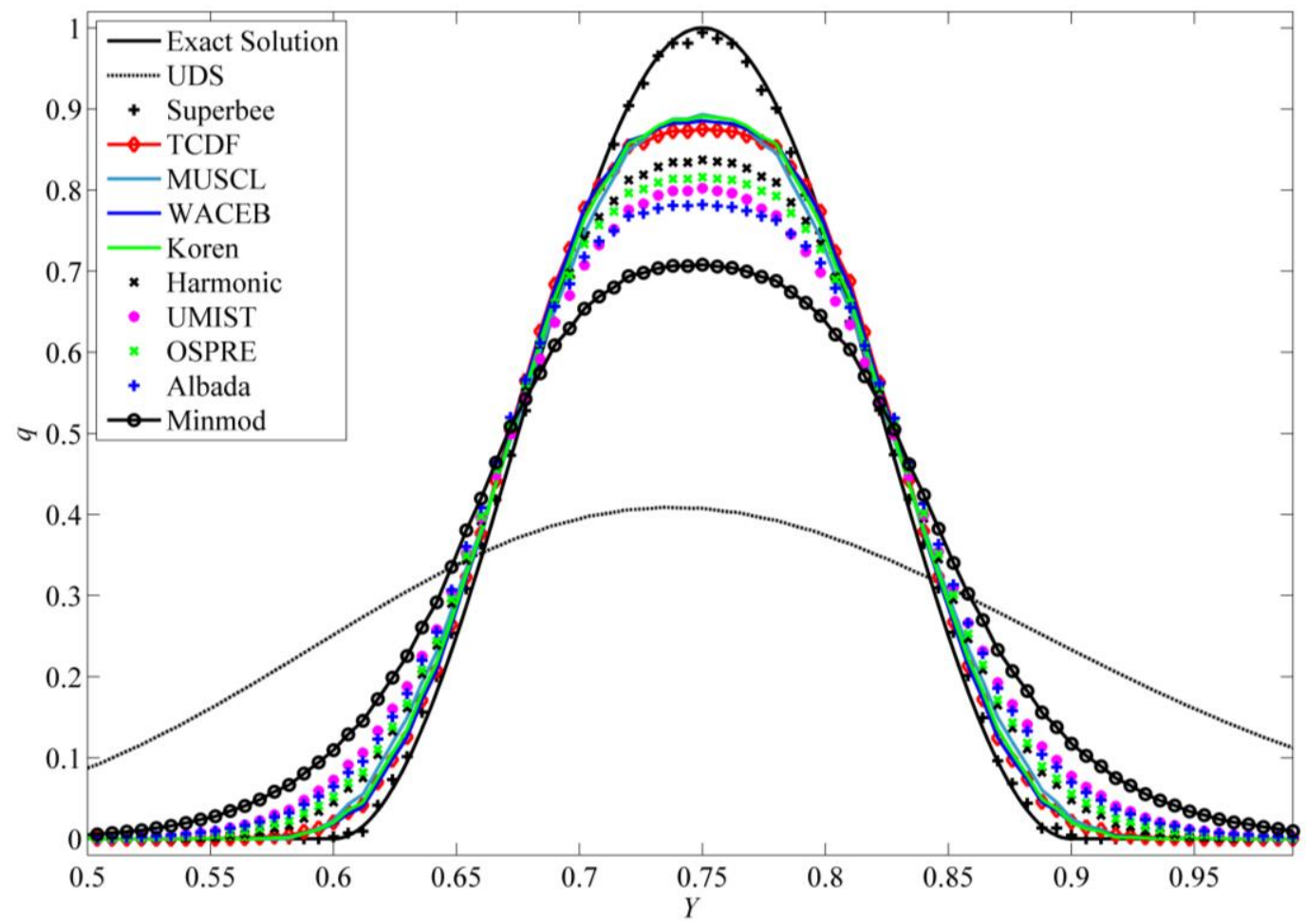

Figure 9. Comparison of accuracy of various SS-TVD flux-limiters at a Courant number of 3.0 in Test 2.

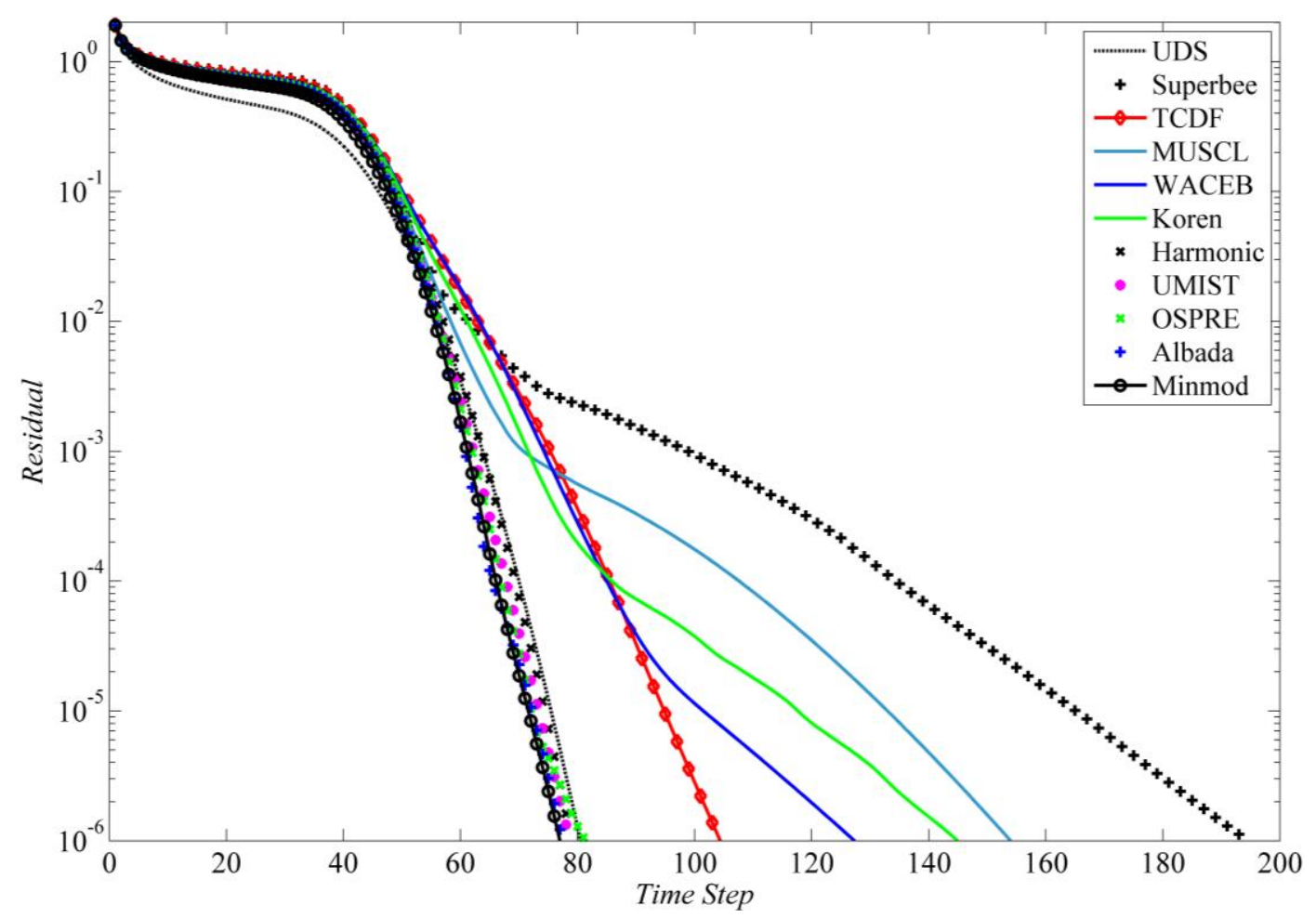

Figure 10. Comparison of convergence of various SS-TVD flux-limiters at a Courant number of 3.0 in Test 2. 


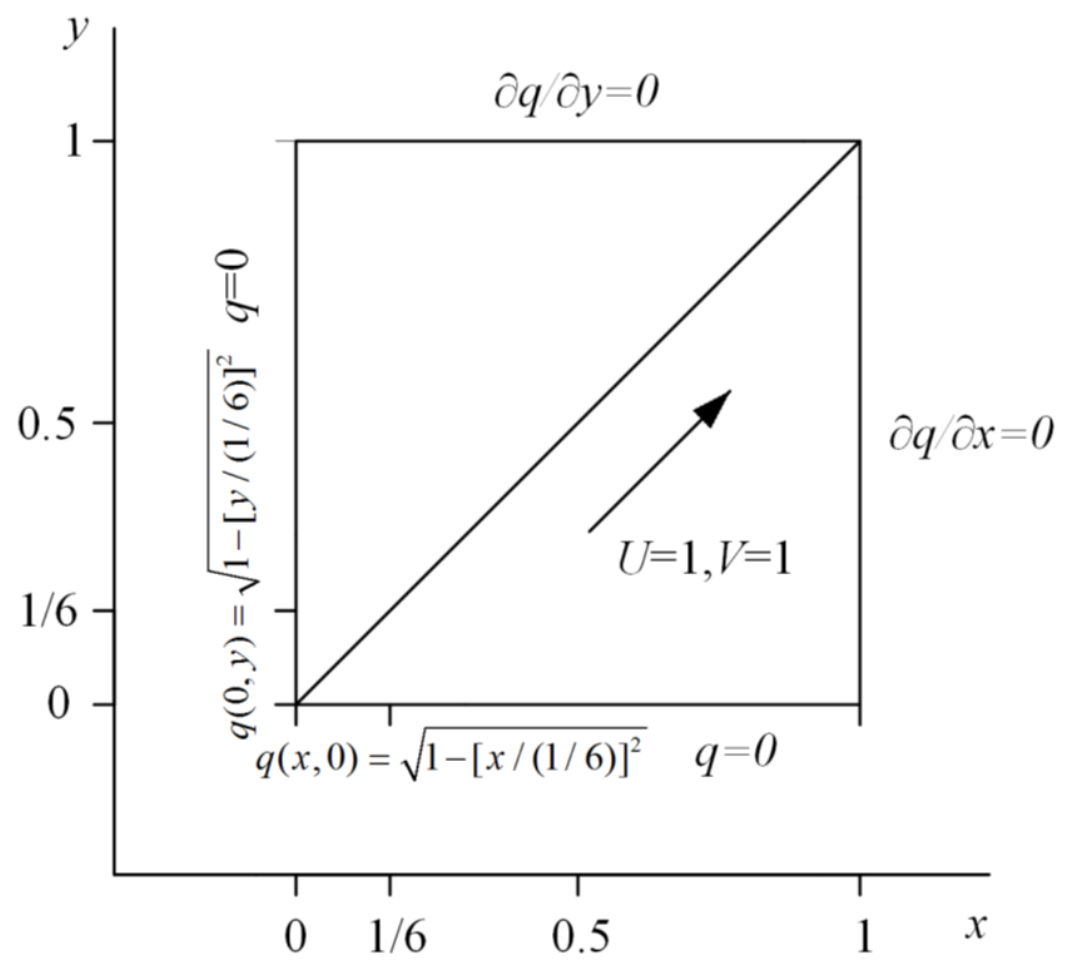

Figure 11. Pure convection of a semi-ellipse profile by a uniform velocity field.

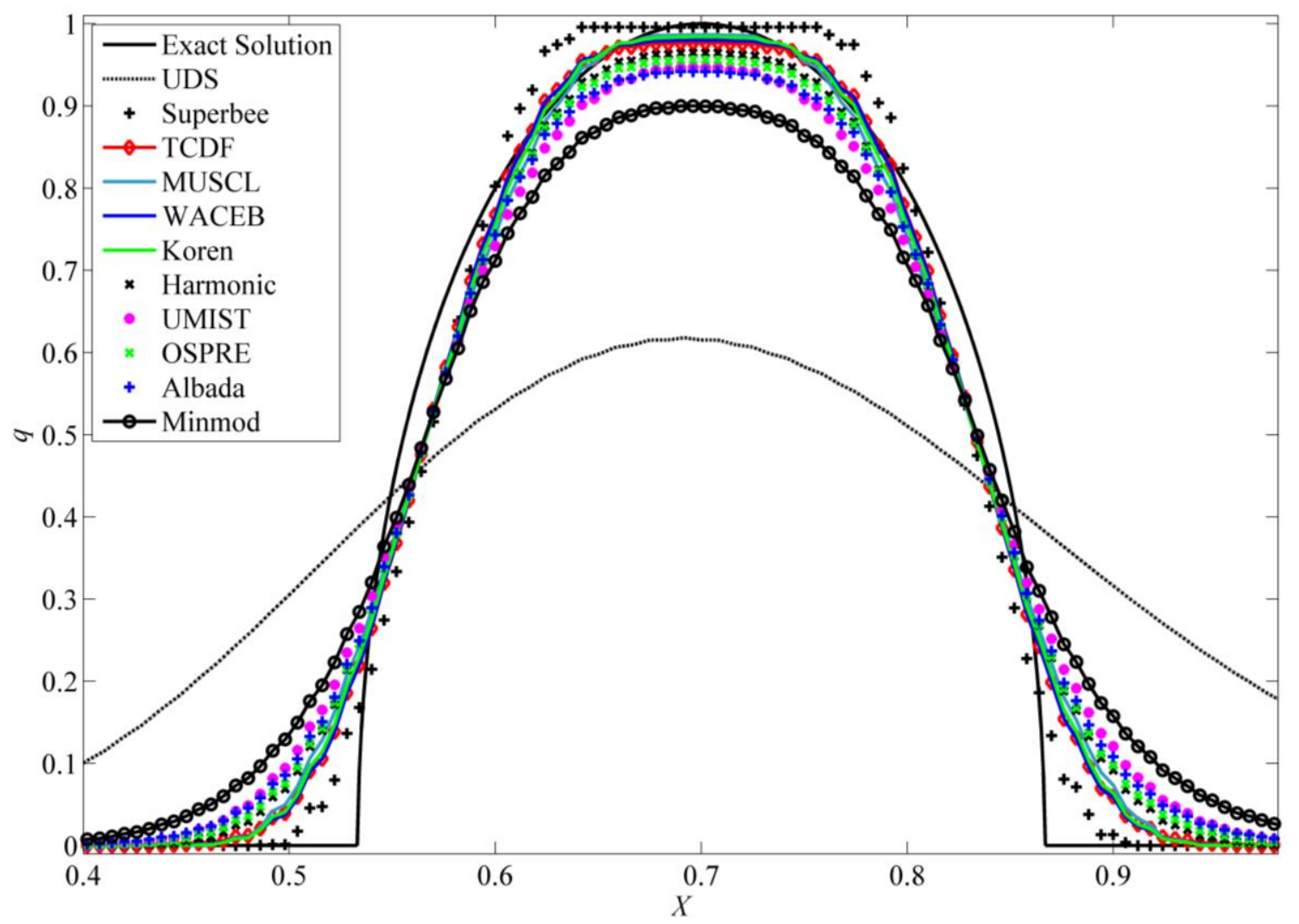

Figure 12. Comparison of accuracy of various SS-TVD flux-limiters at a Courant number of 3.0 in Test 3. 


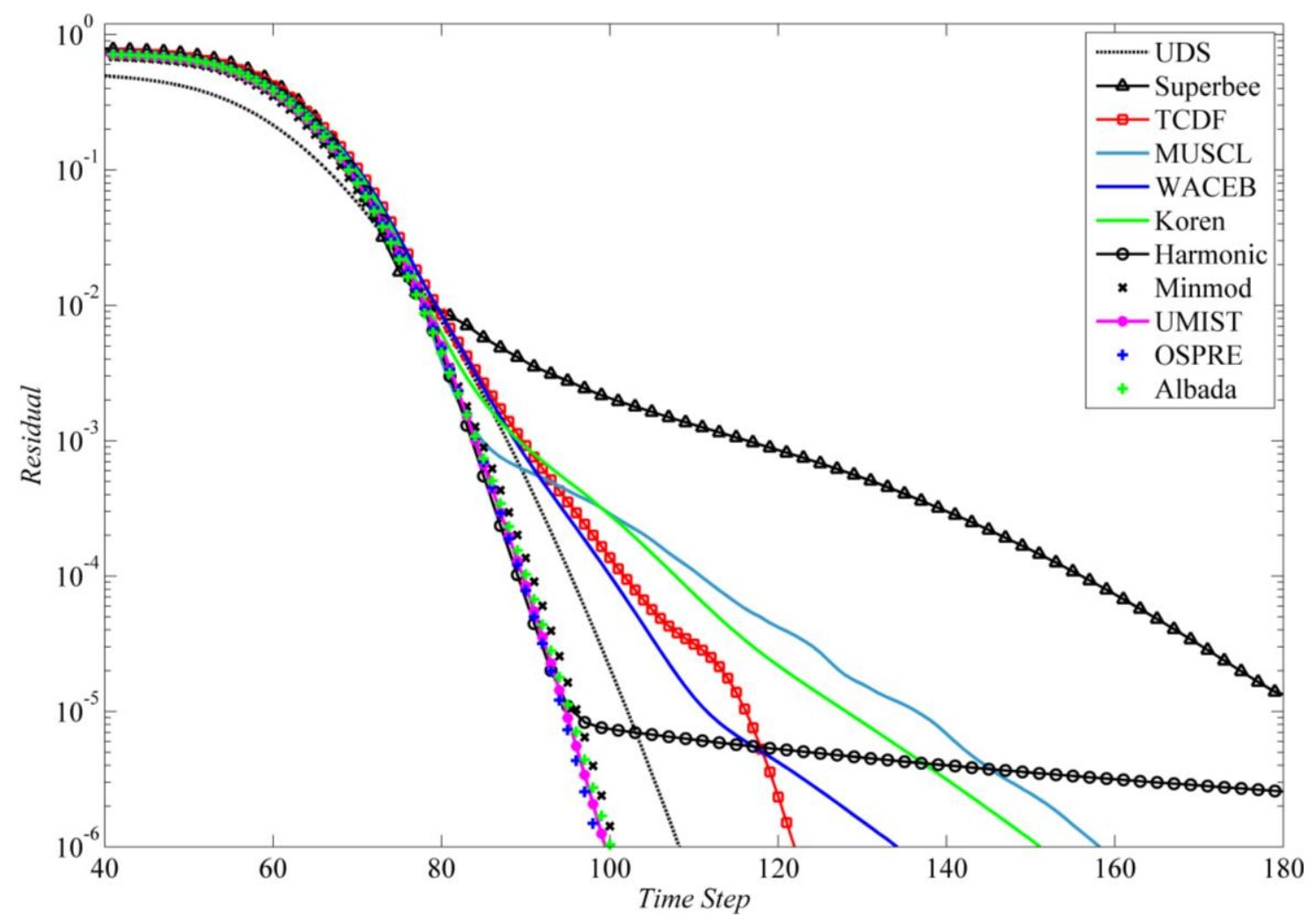

Figure 13. Comparison of convergence of various SS-TVD flux-limiters at a Courant number of 3.0 in Test 3.

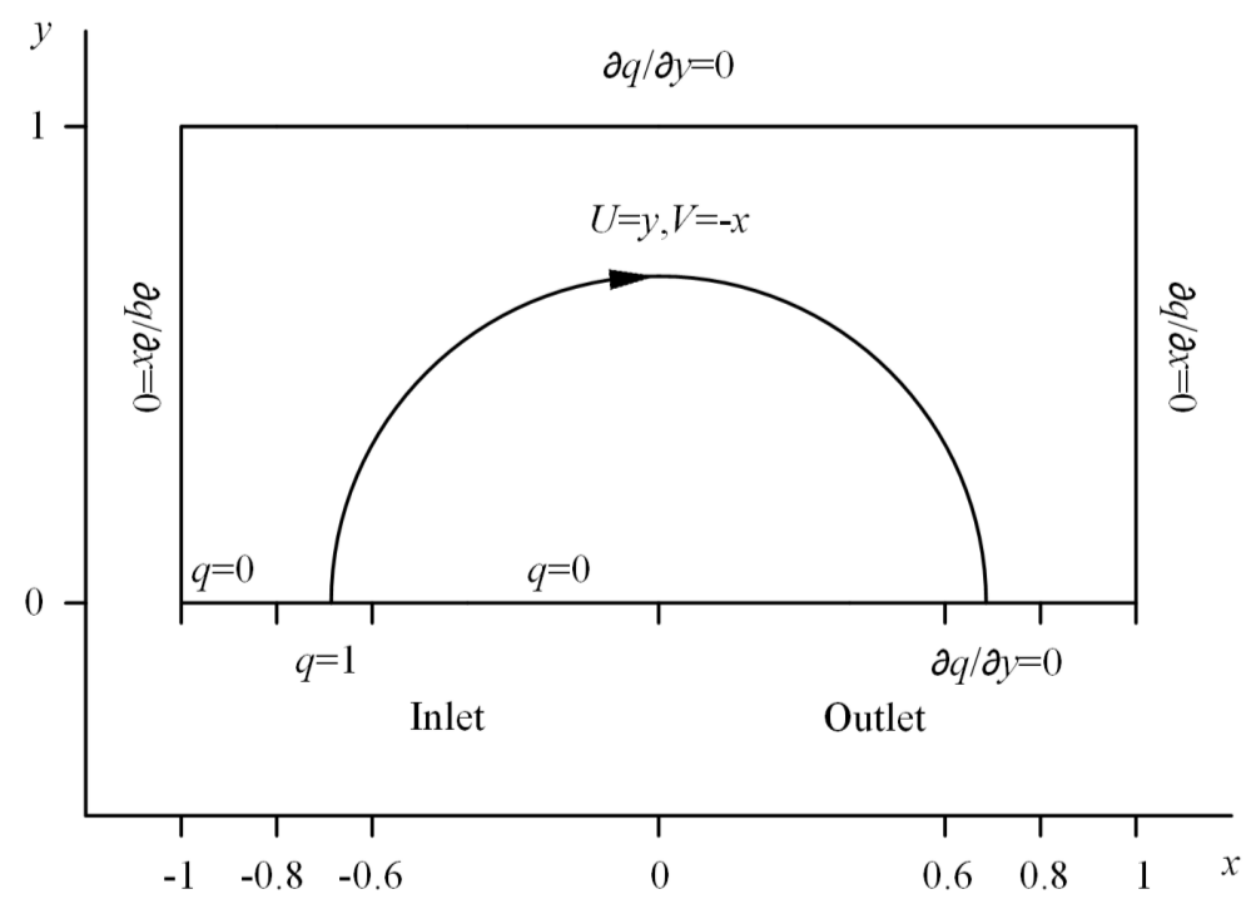

Figure 14. Pure convection of a one-double-step profile in a rotational velocity field. 


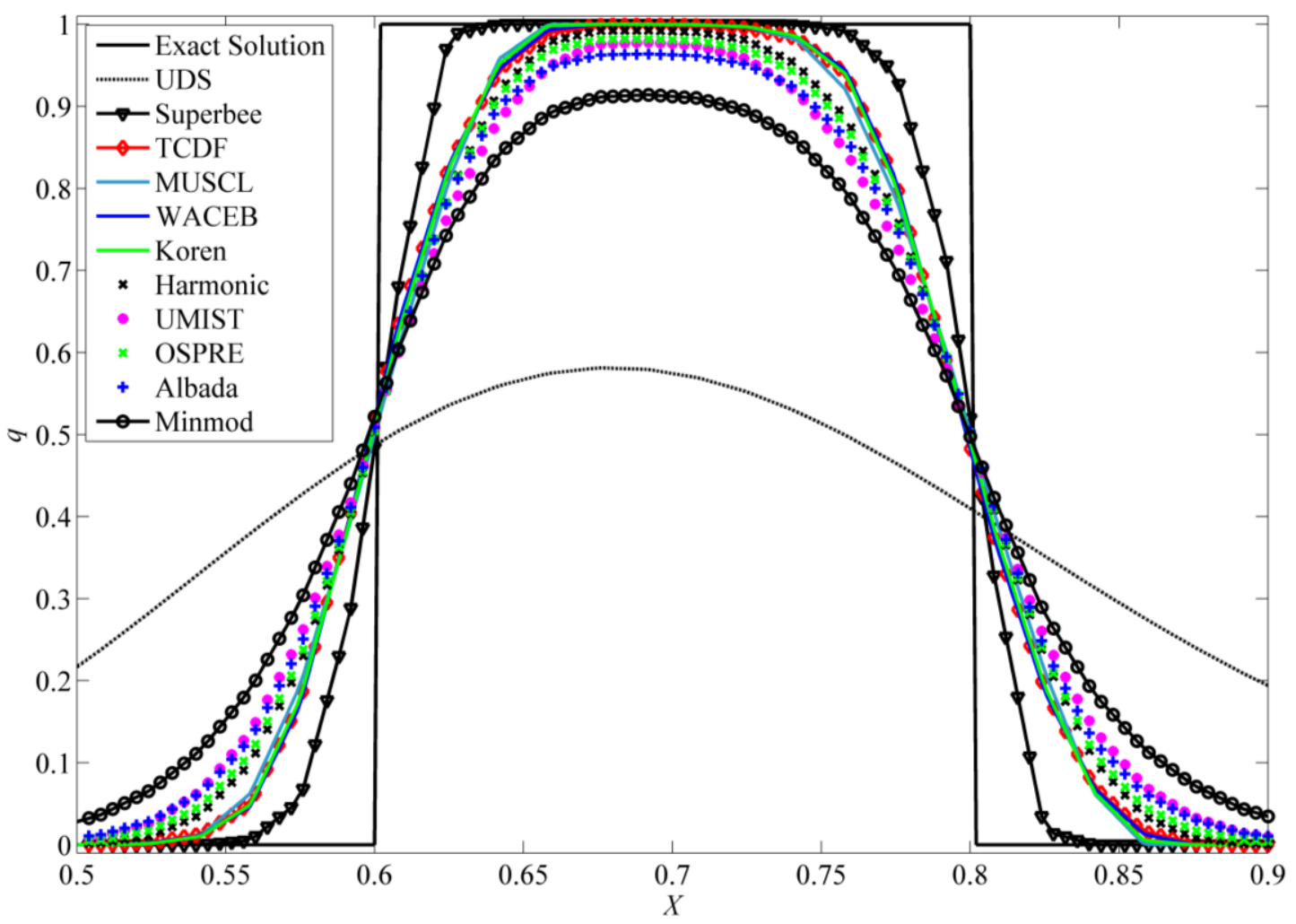

Figure 15. Comparison of accuracy of various SS-TVD flux-limiters at a Courant number of 4.0 in Test 4.

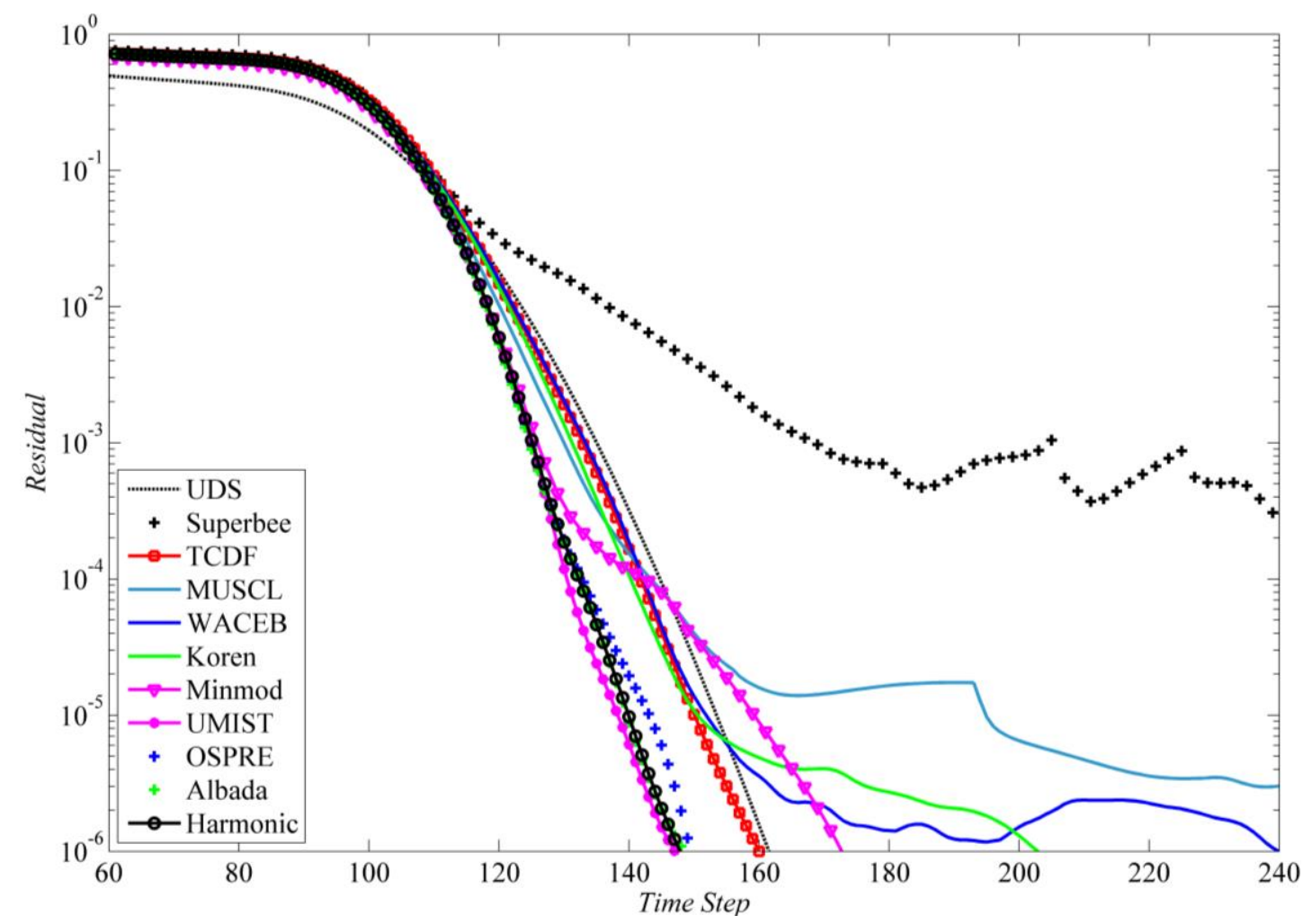

Figure 16. Comparison of convergence of various SS-TVD flux-limiters at a Courant number of 4.0 in Test 4. 


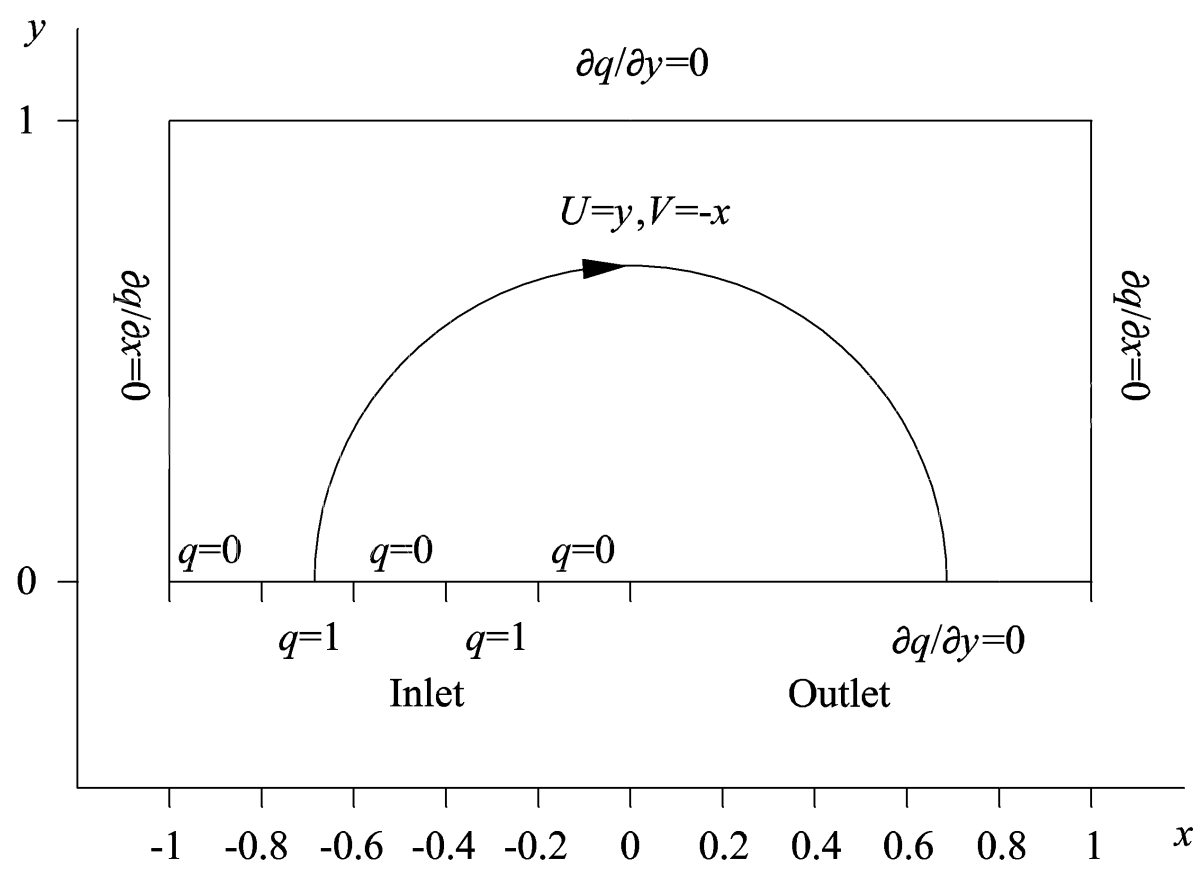

Figure 17. Pure convection of a two-double-step profile in a rotational velocity field.

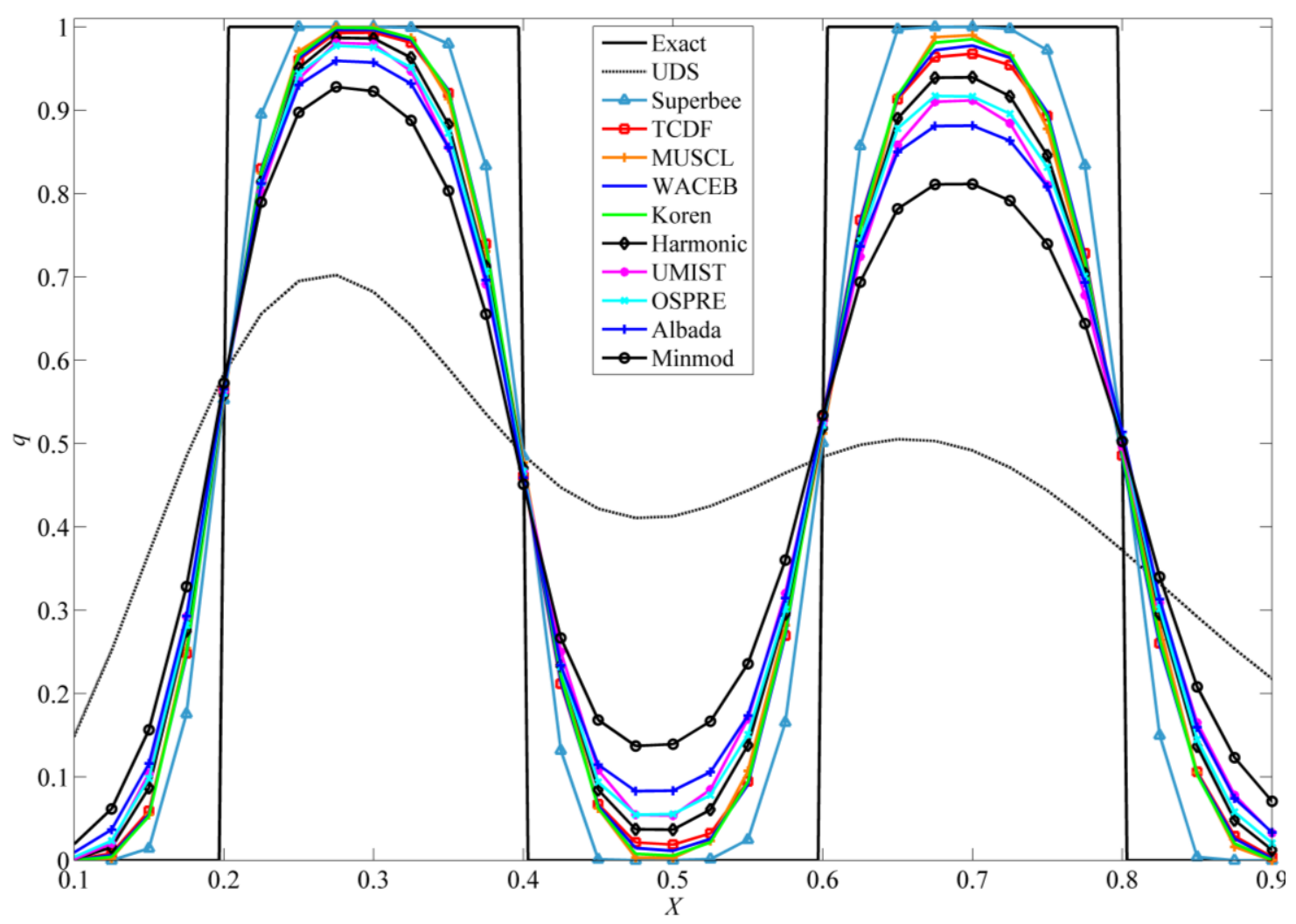

Figure 18. Comparison of accuracy of various SS-TVD flux-limiters for the mesh composed of $40 \times 80$ cells at a Courant number of 3.0 in Test 5 . 


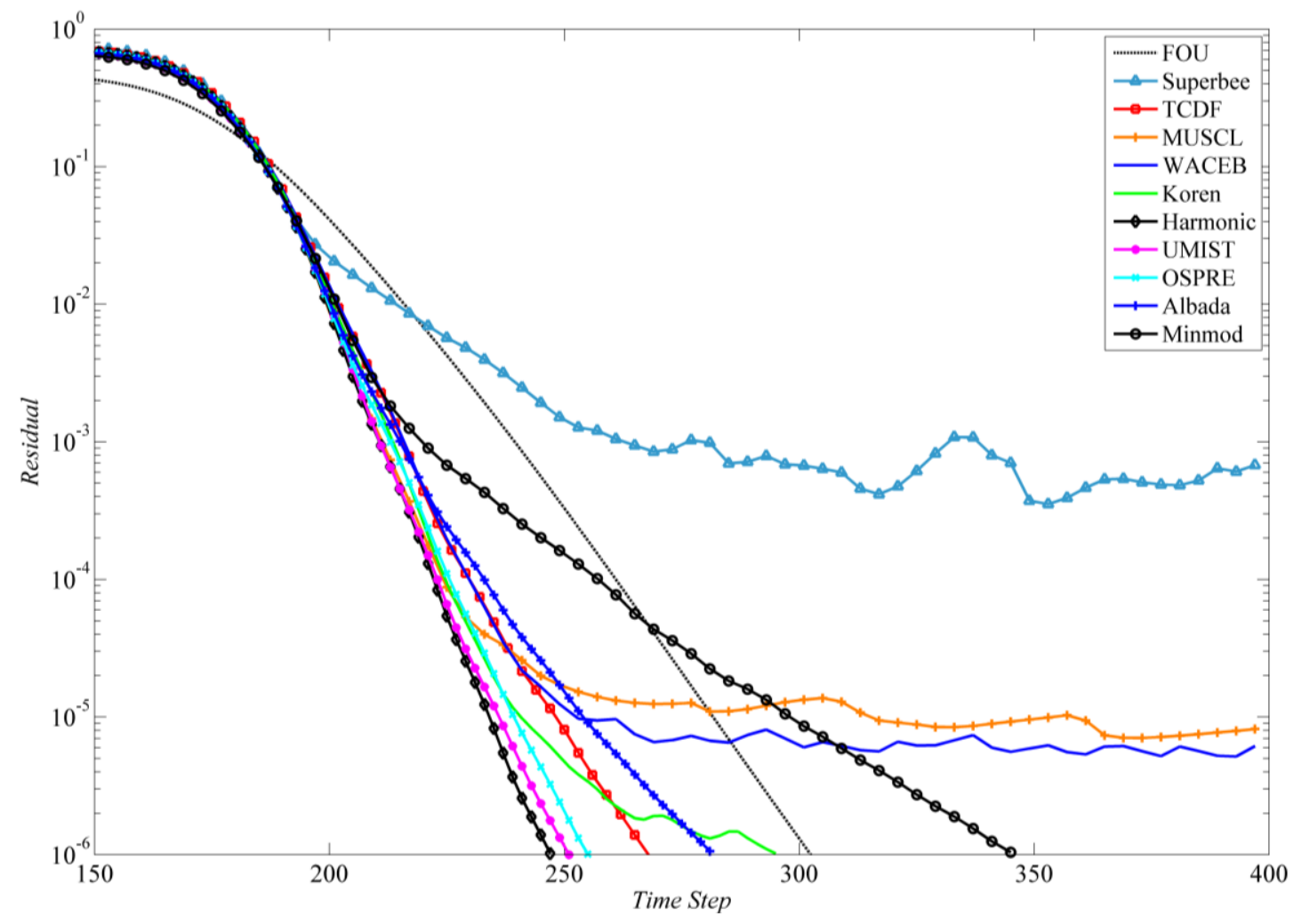

Figure 19. Comparison of convergence of various SS-TVD flux-limiters for the mesh composed of $80 \times 160$ cells at a Courant number of 3.0 in Test 5 .

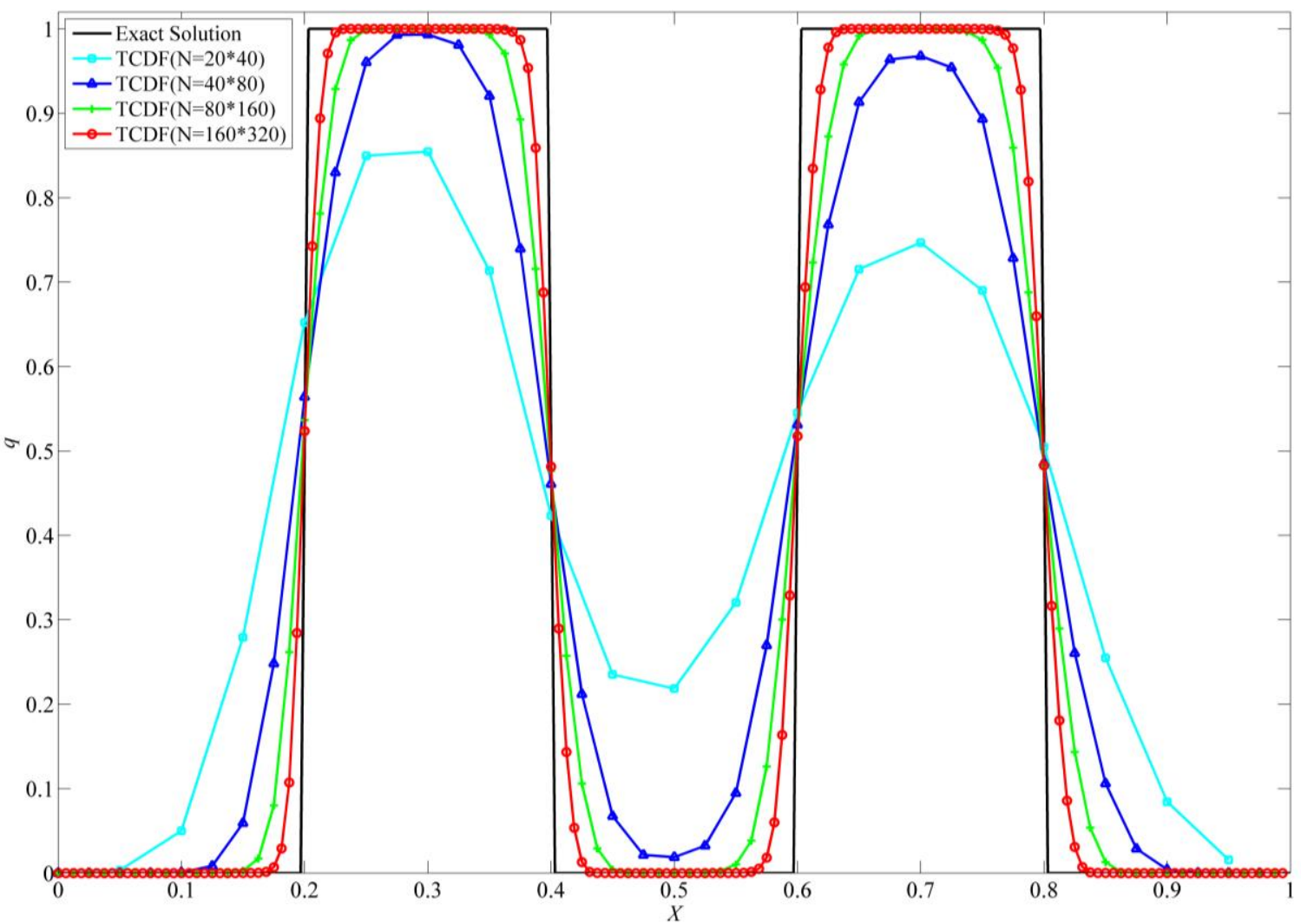

Figure 20. The resulting profiles at the outlet plane for the TCDF limiter on various mesh resolutions at a Courant number of 3.0 in Test 5. 


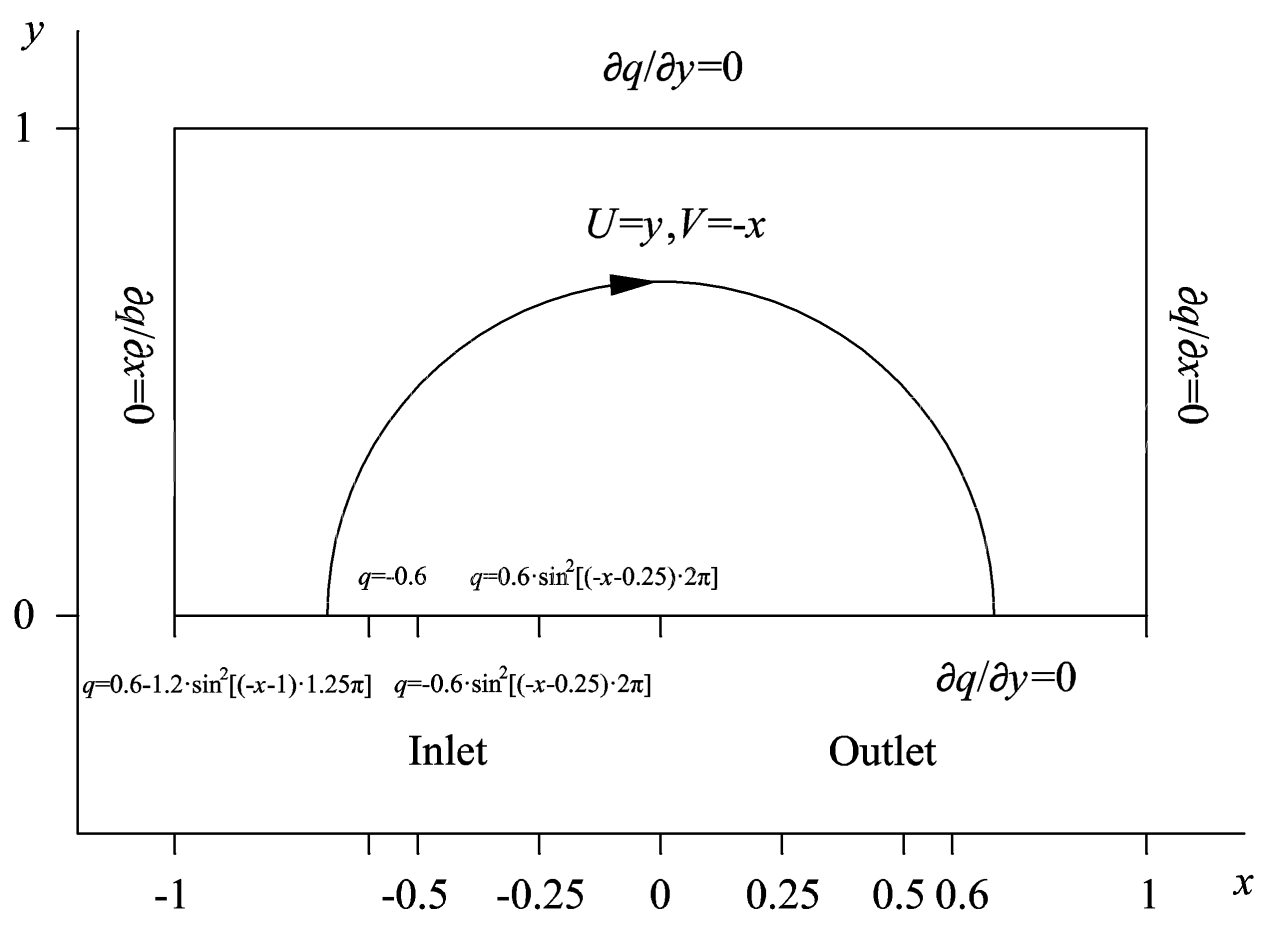

Figure 21. Pure convection of a relatively smooth profile in a rotational velocity field.

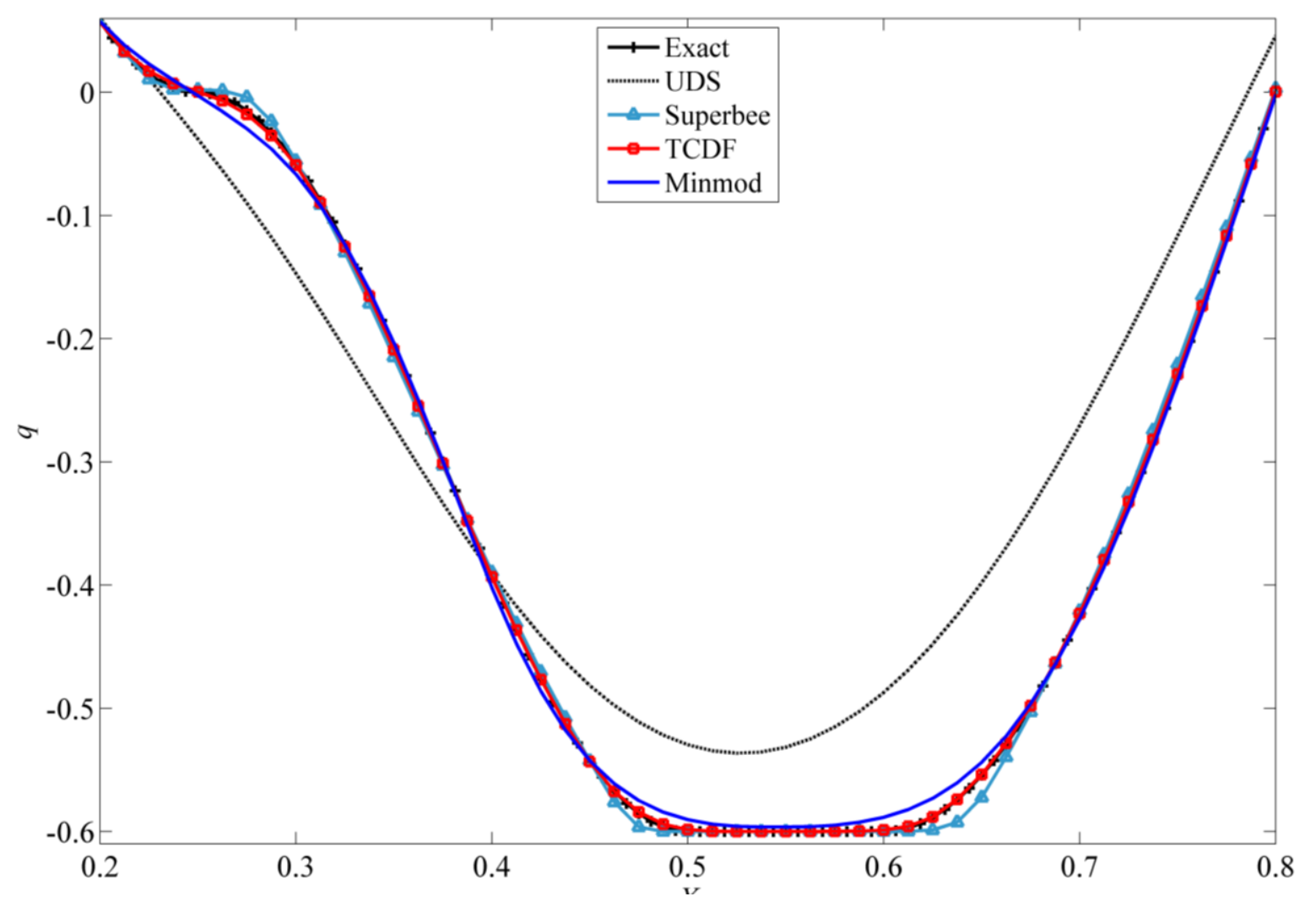

Figure 22. Comparison of accuracy of various SS-TVD flux-limiters for the mesh composed of $80 \times 160$ cells at a Courant number of 1.0 in Test 6 . 


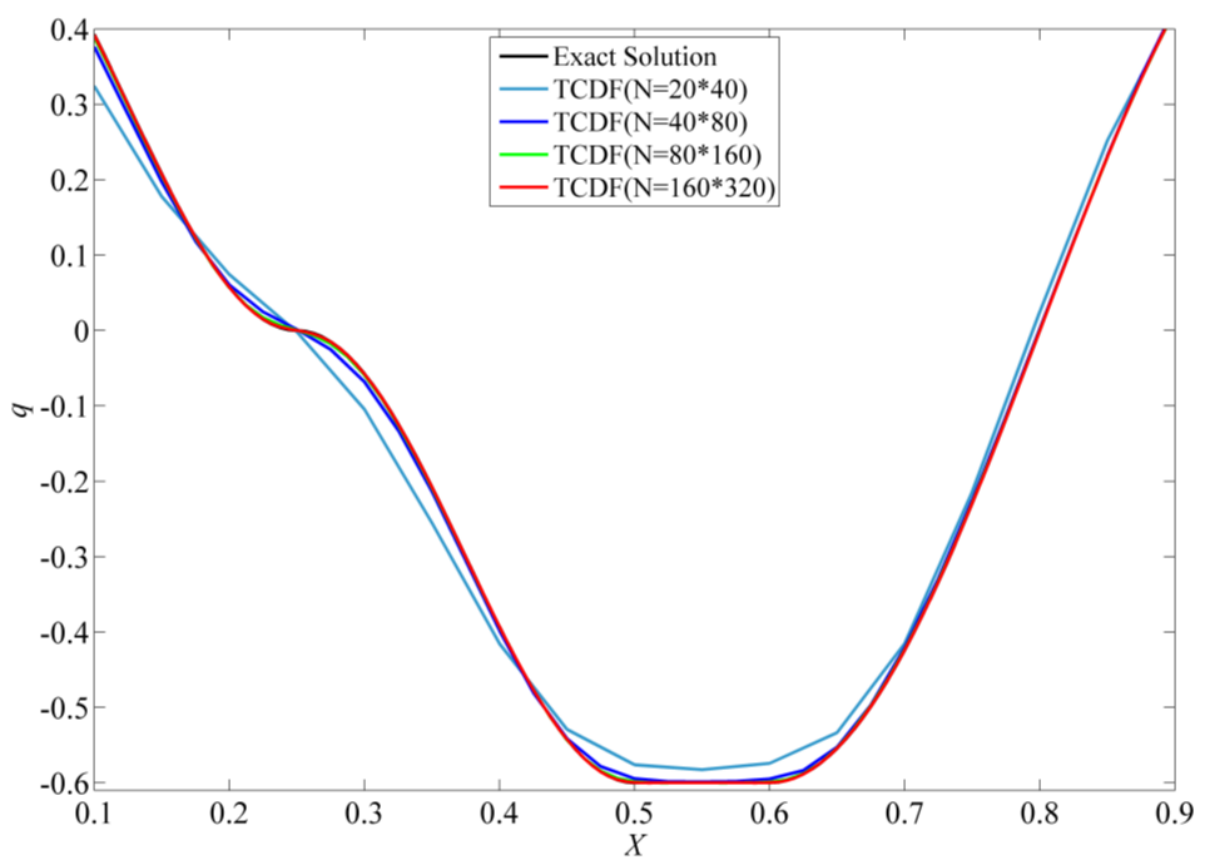

Figure 23. The resulting profiles at the outlet plane for the TCDF limiter on various mesh resolutions at a Courant number of 1.0 in Test 6 .

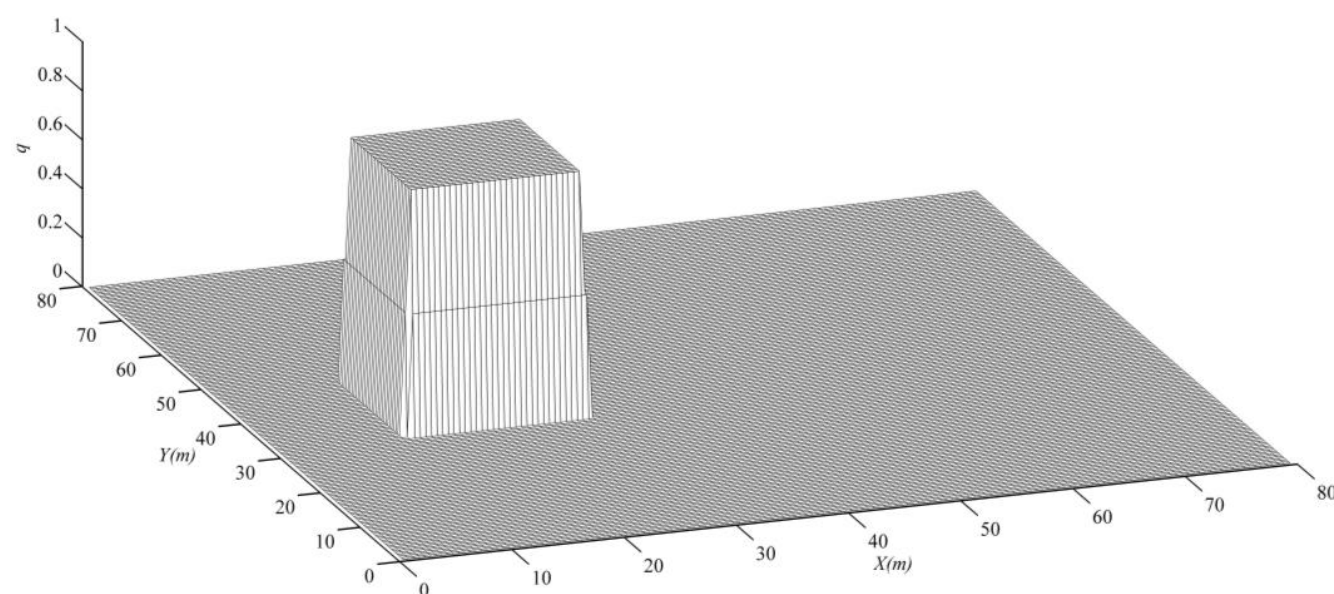

Figure 24. The initial concentration distribution in Test 7.

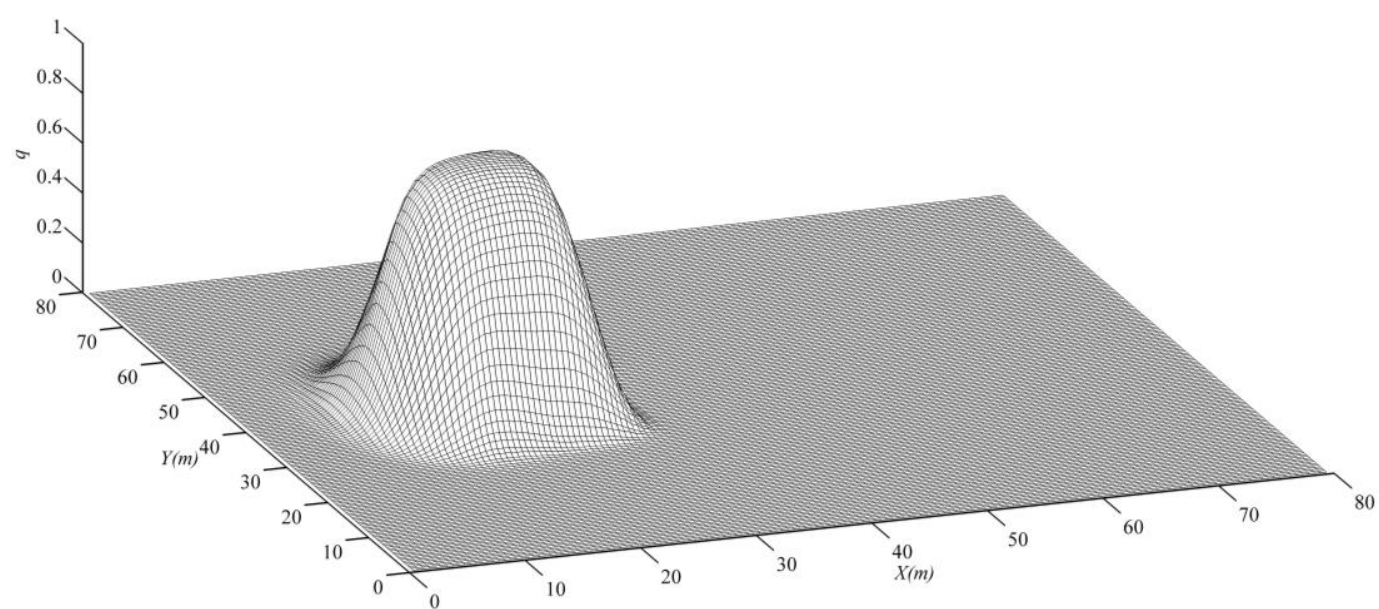

Figure 25. The resulting profile after one rotation for the Harmonic limiter on the mesh consisting of $80 \times 80$ cells at a time step of 0.1 in Test 7 . 


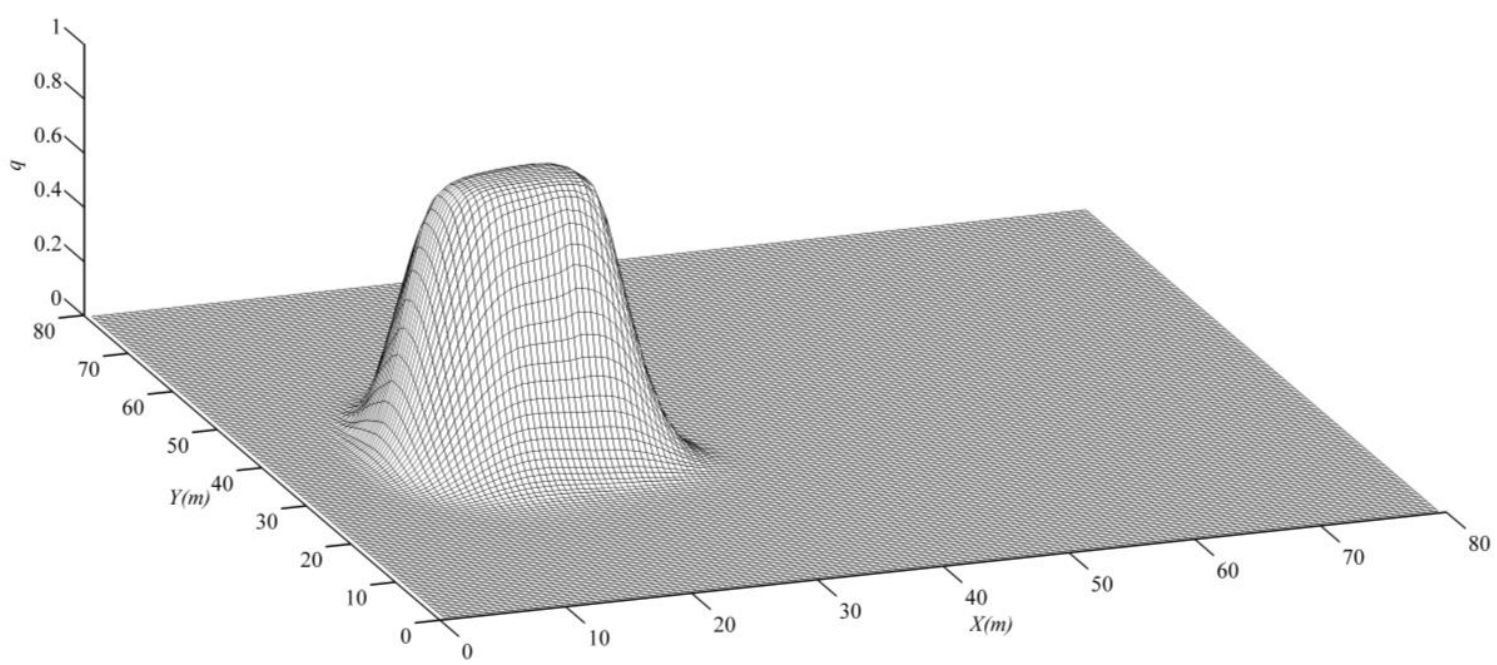

Figure 26. The resulting profile after one rotation for the TCDF limiter on the mesh consisting of $80 \times 80$ cells at a time step of 0.1 in Test 7 .

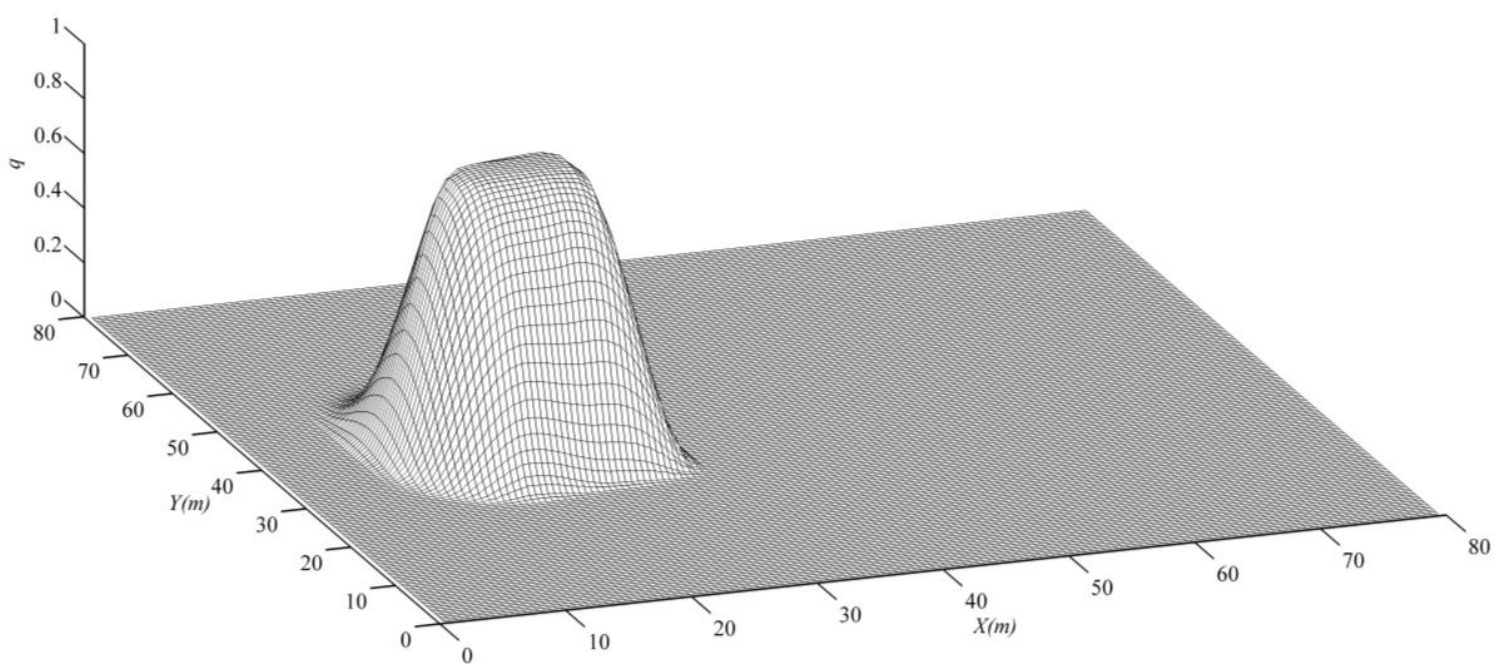

Figure 27. The resulting profile after one rotation for the MUSCL limiter on the mesh consisting of $80 \times 80$ cells at a time step of 0.1 in Test 7 .

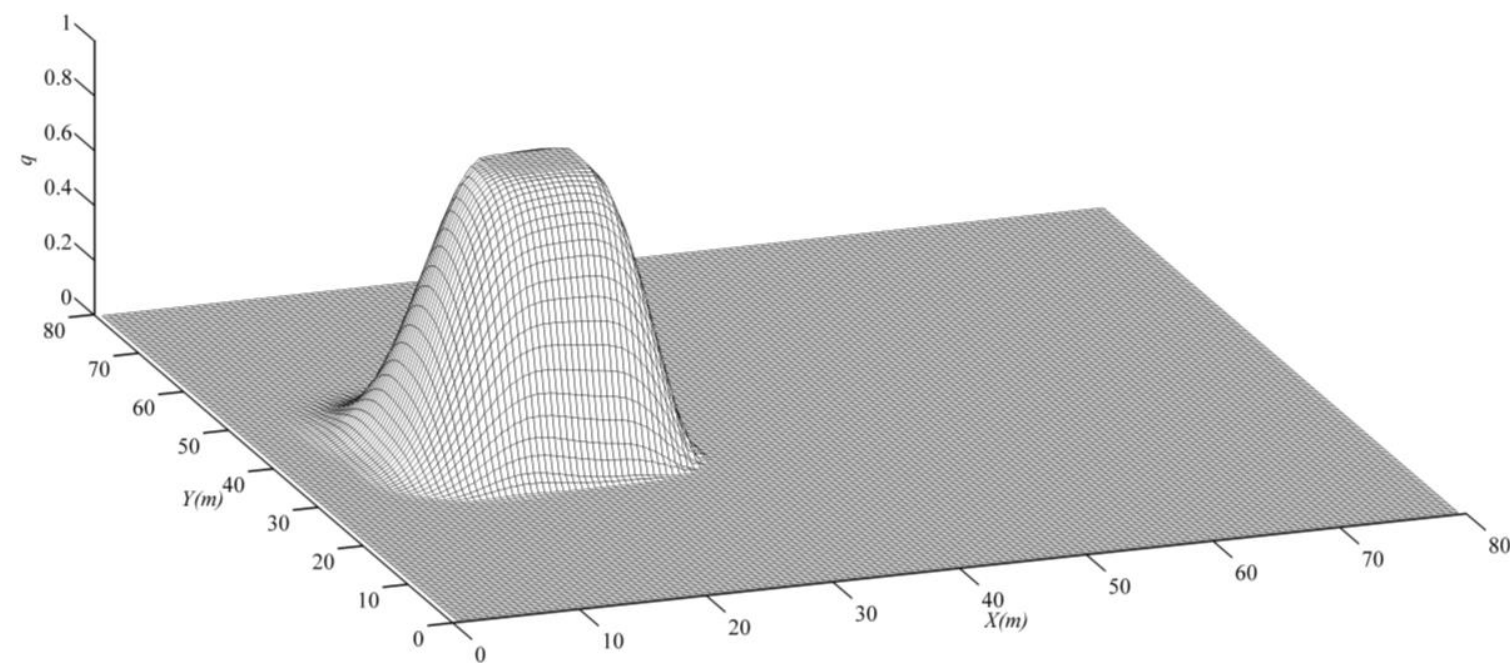

Figure 28. The resulting profile after one rotation for the CFL-MUSCL limiter on the mesh consisting of $80 \times 80$ cells at a time step of 0.1 in Test 7 . 


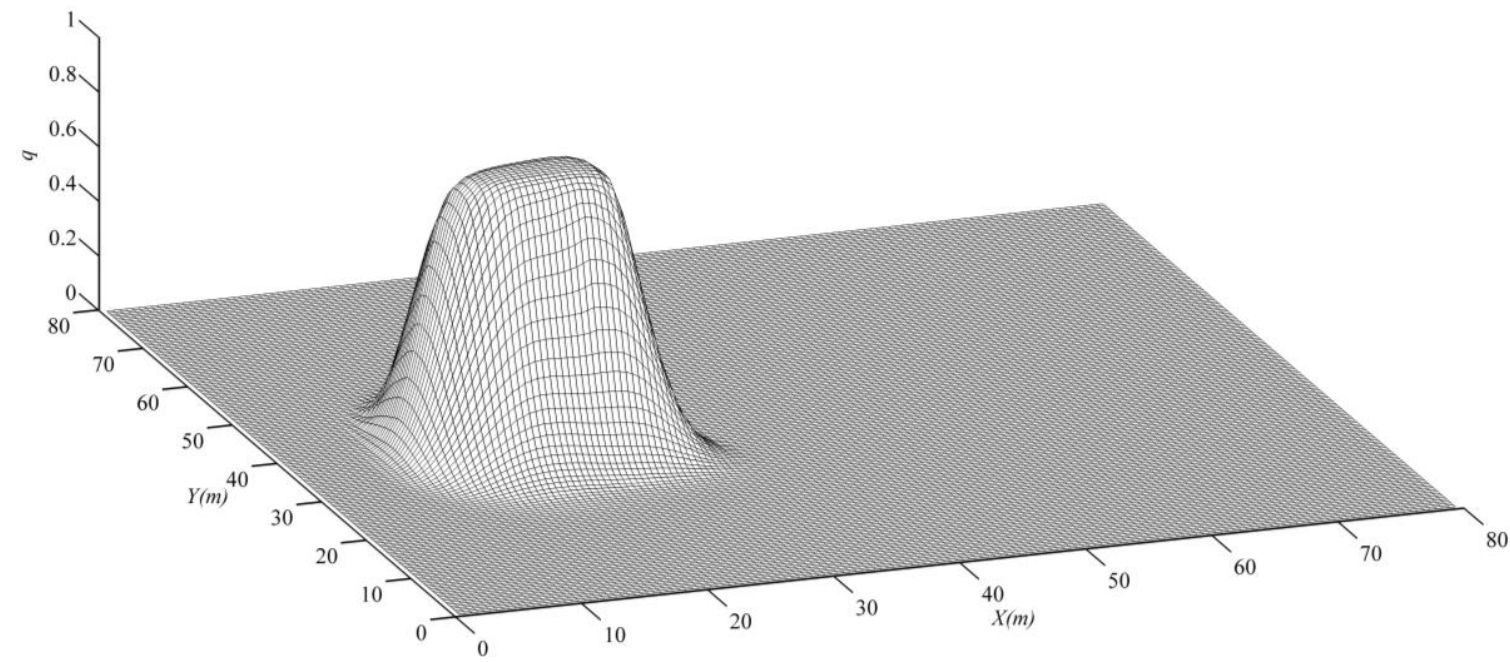

Figure 29. The resulting profile after one rotation for the WACEB limiter on the mesh consisting of $80 \times 80$ cells at a time step of 0.1 in Test 7 .

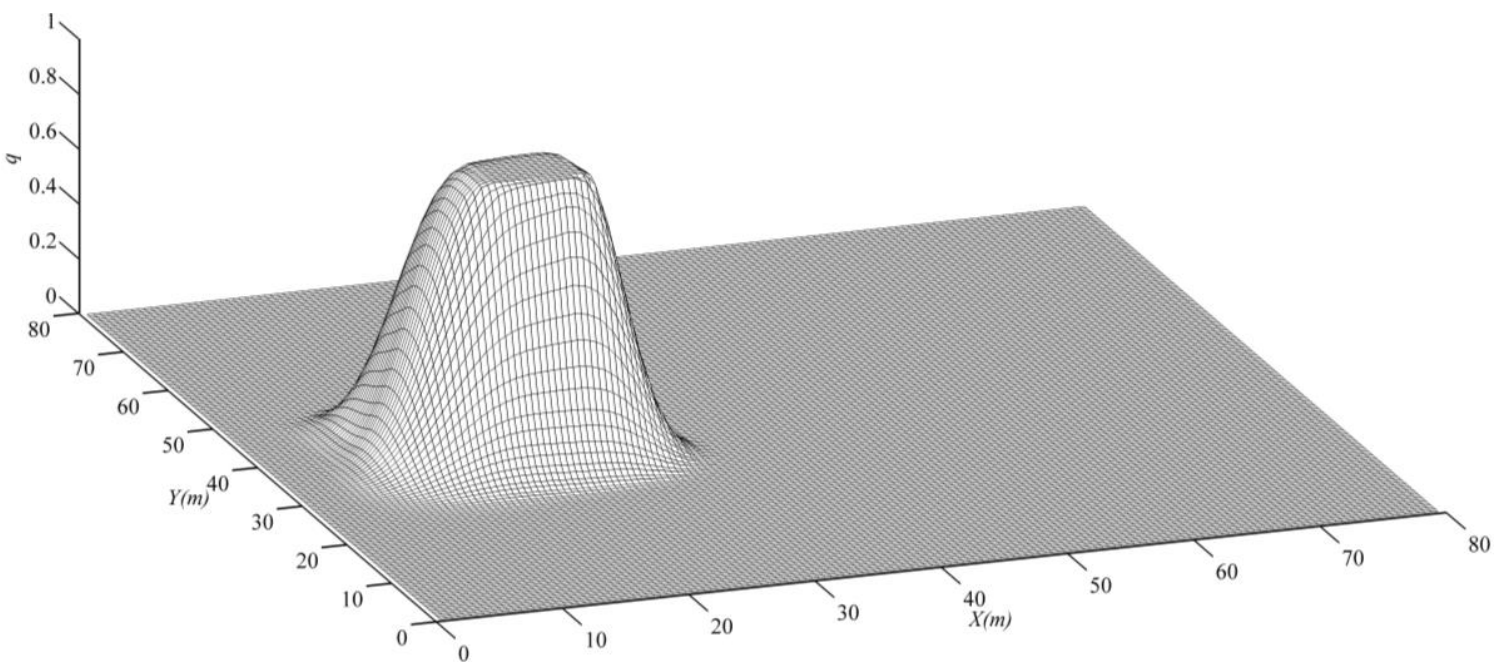

Figure 30. The resulting profile after one rotation for the CFL-WACEB limiter on the mesh consisting of $80 \times 80$ cells at a time step of 0.1 in Test 7 .

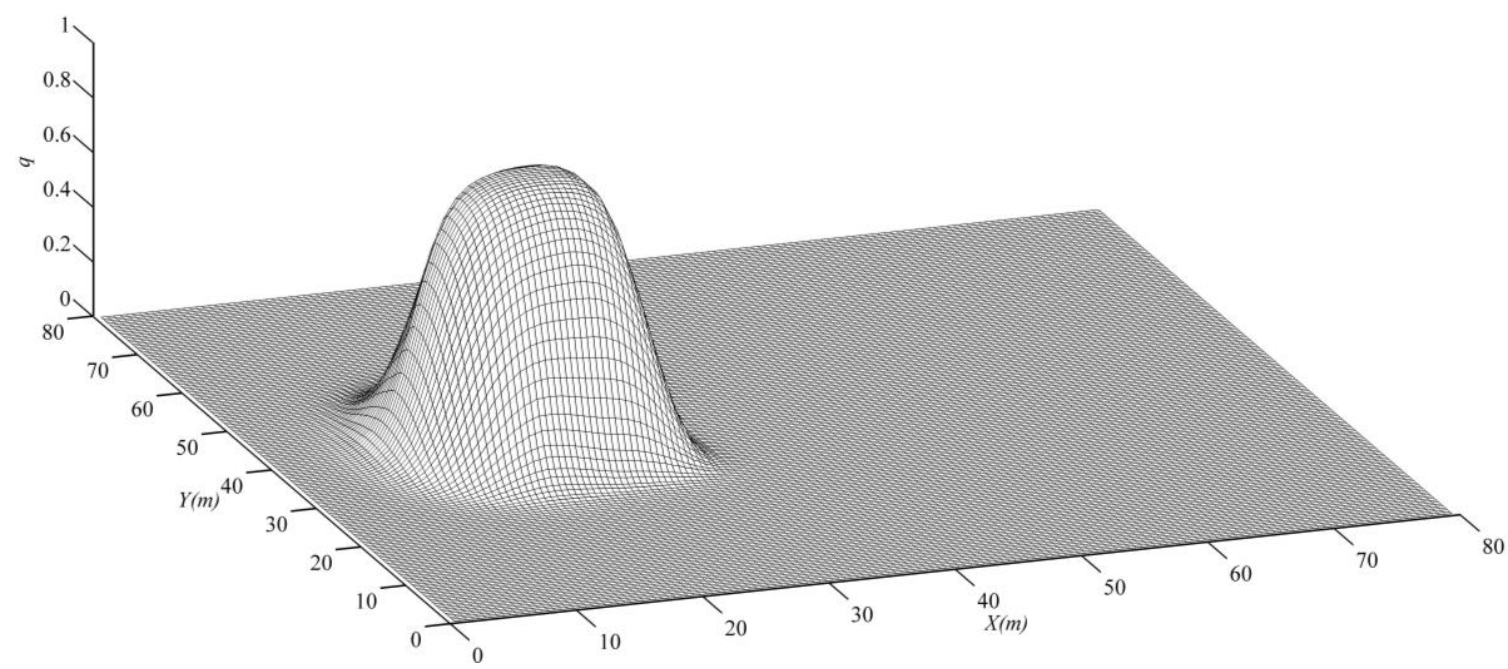

Figure 31. The resulting profile after one rotation for the OSPRE limiter on the mesh consisting of $80 \times 80$ cells at a time step of 0.1 in Test 7 . 


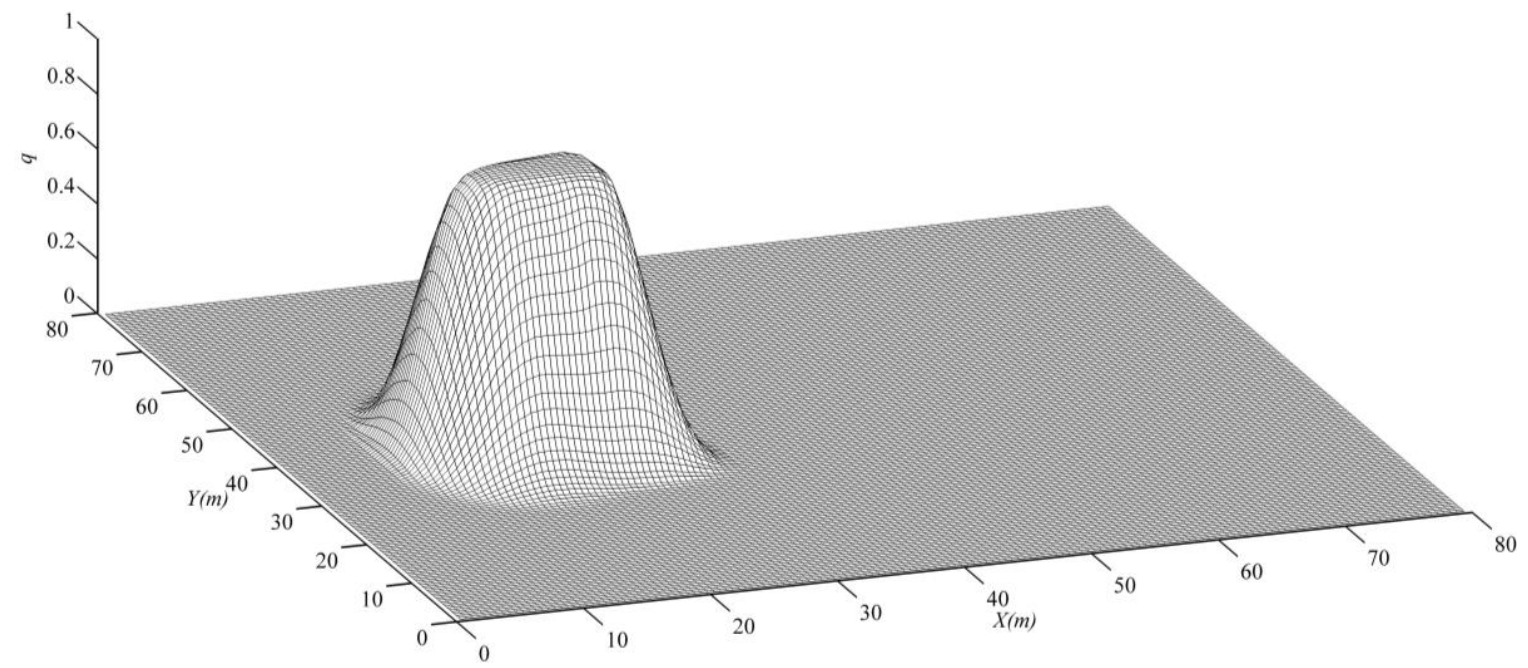

Figure 32. The resulting profile after one rotation for the Koren limiter on the mesh consisting of $80 \times 80$ cells at a time step of 0.1 in Test 7 .

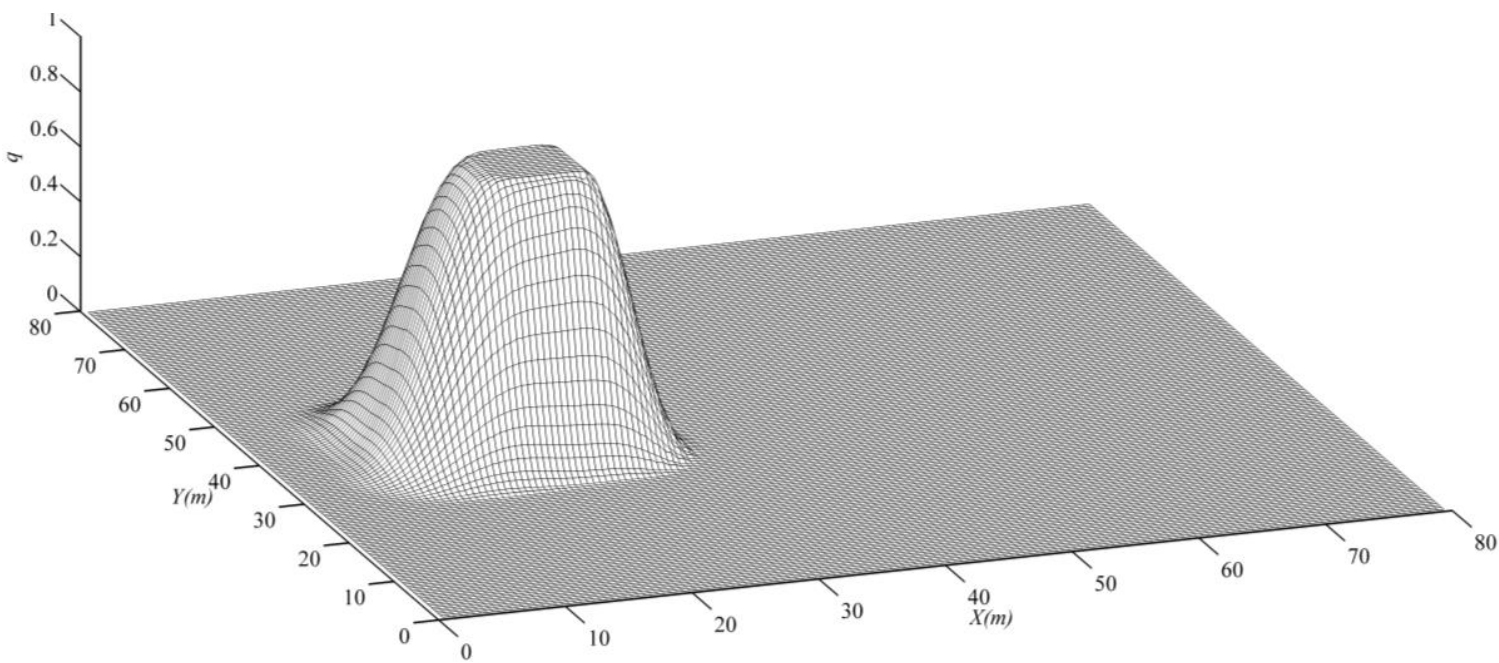

Figure 33. The resulting profile after one rotation for the CFL-Koren limiter on the mesh consisting of $80 \times 80$ cells at a time step of 0.1 in Test 7 .

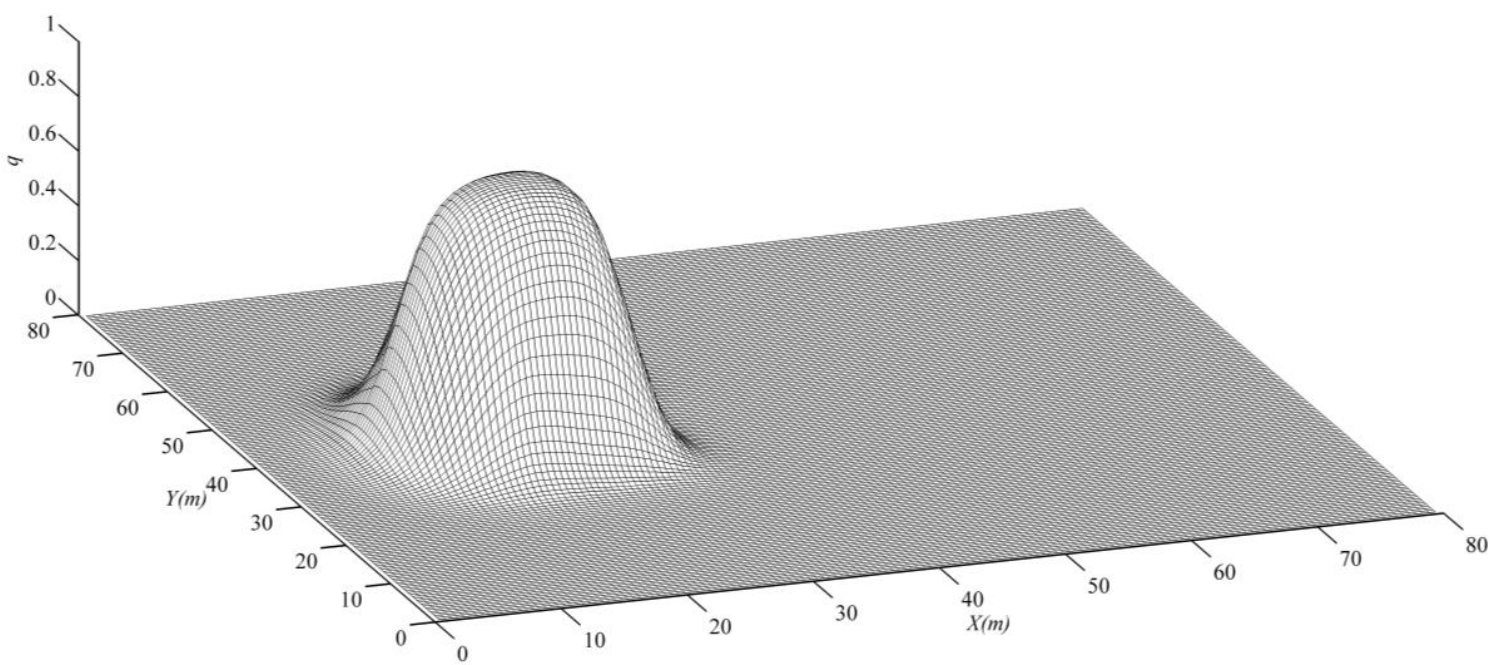

Figure 34. The resulting profile after one rotation for the Albada limiter on the mesh consisting of $80 \times 80$ cells at a time step of 0.1 in Test 7 . 


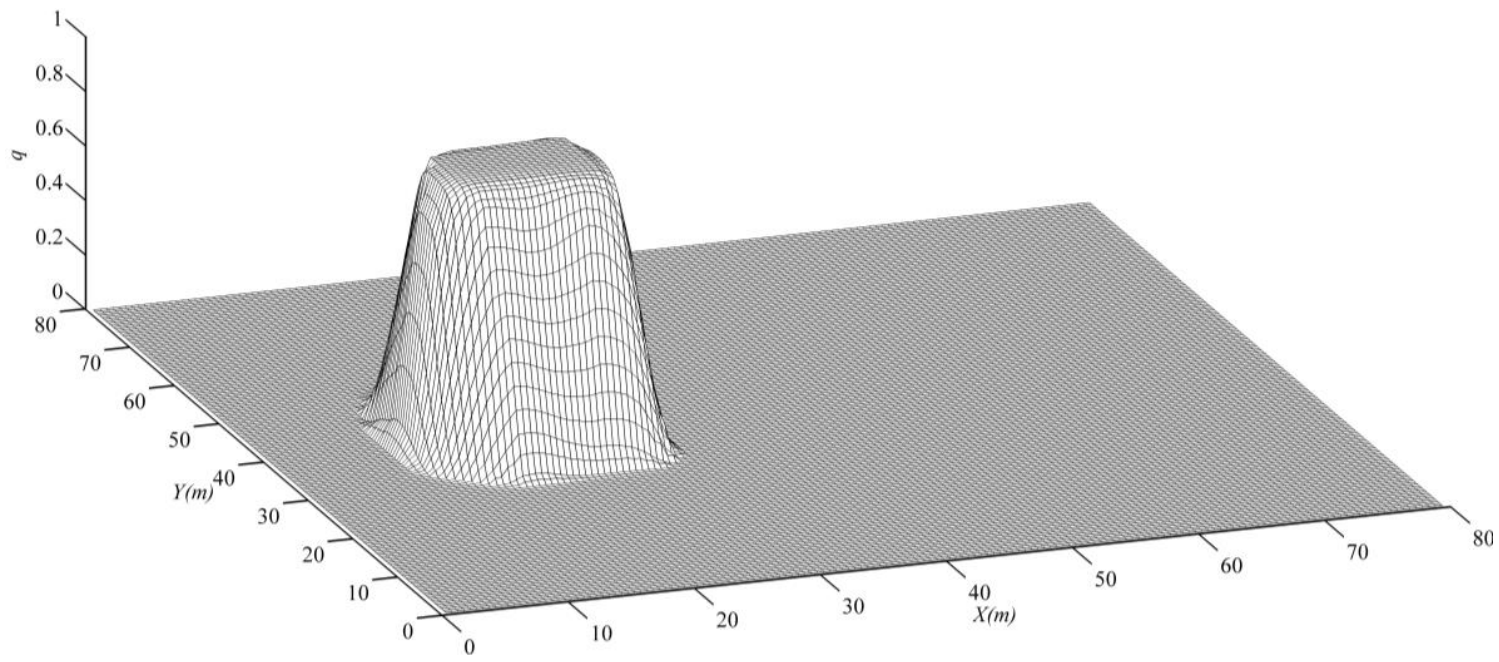

Figure 35. The resulting profile after one rotation for the Superbee limiter on the mesh consisting of $80 \times 80$ cells at a time step of 0.1 in Test 7 .

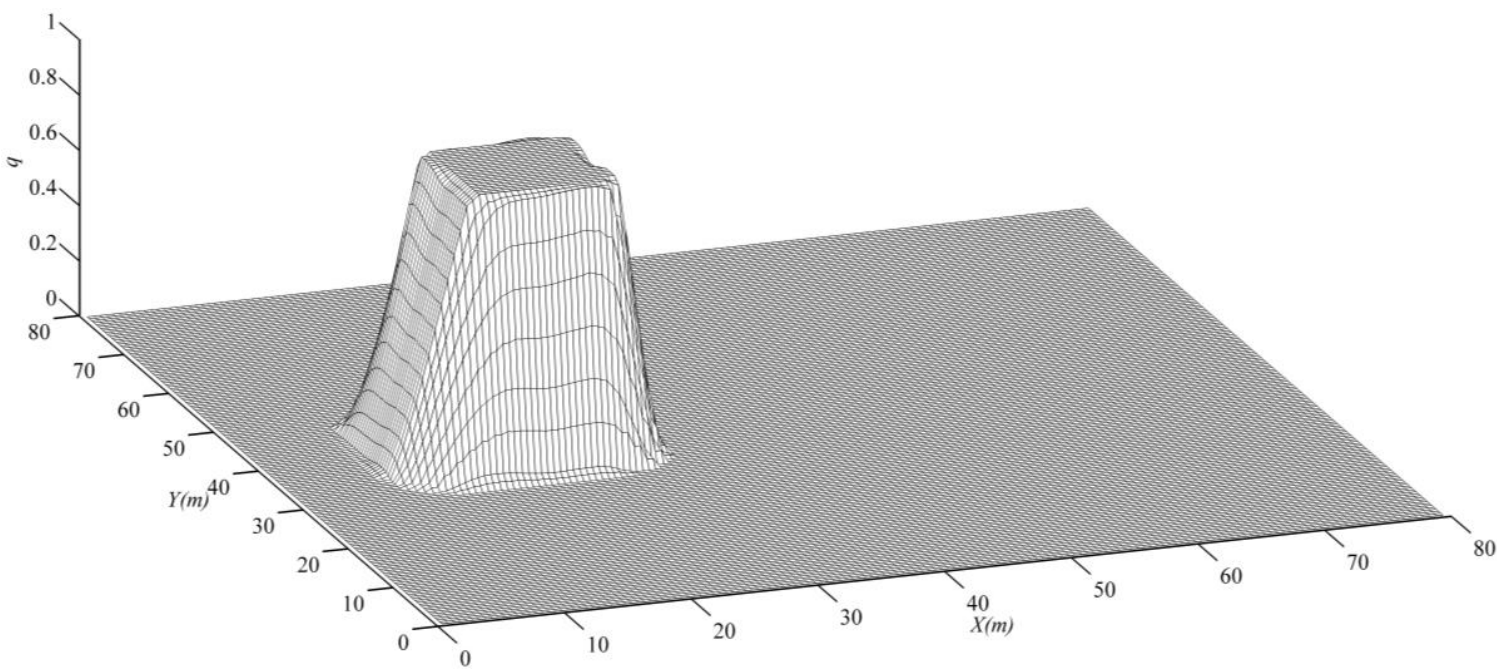

Figure 36. The resulting profile after one rotation for the CFL-Superbee limiter on the mesh consisting of $80 \times 80$ cells at a time step of 0.1 in Test 7 .

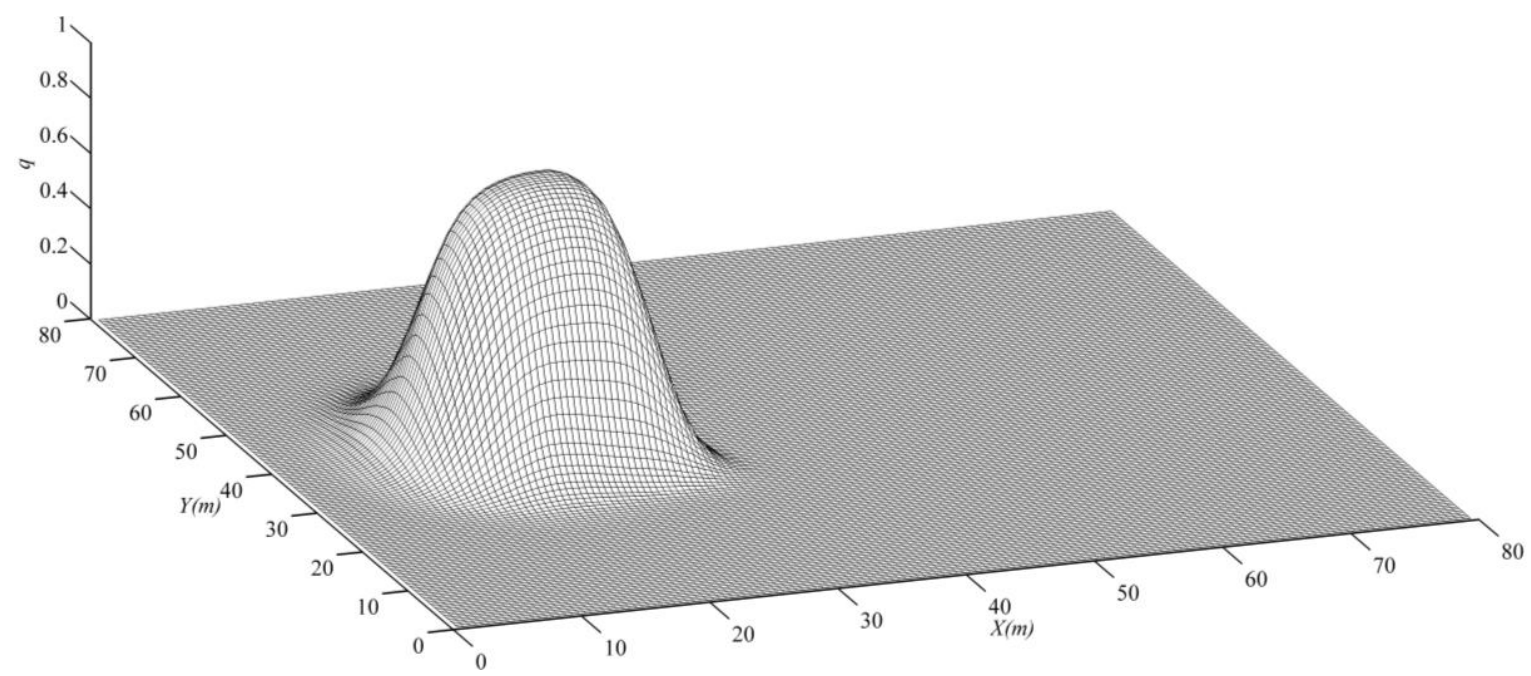

Figure 37. The resulting profile after one rotation for the UMIST limiter on the mesh consisting of $80 \times 80$ cells at a time step of 0.1 in Test 7 . 


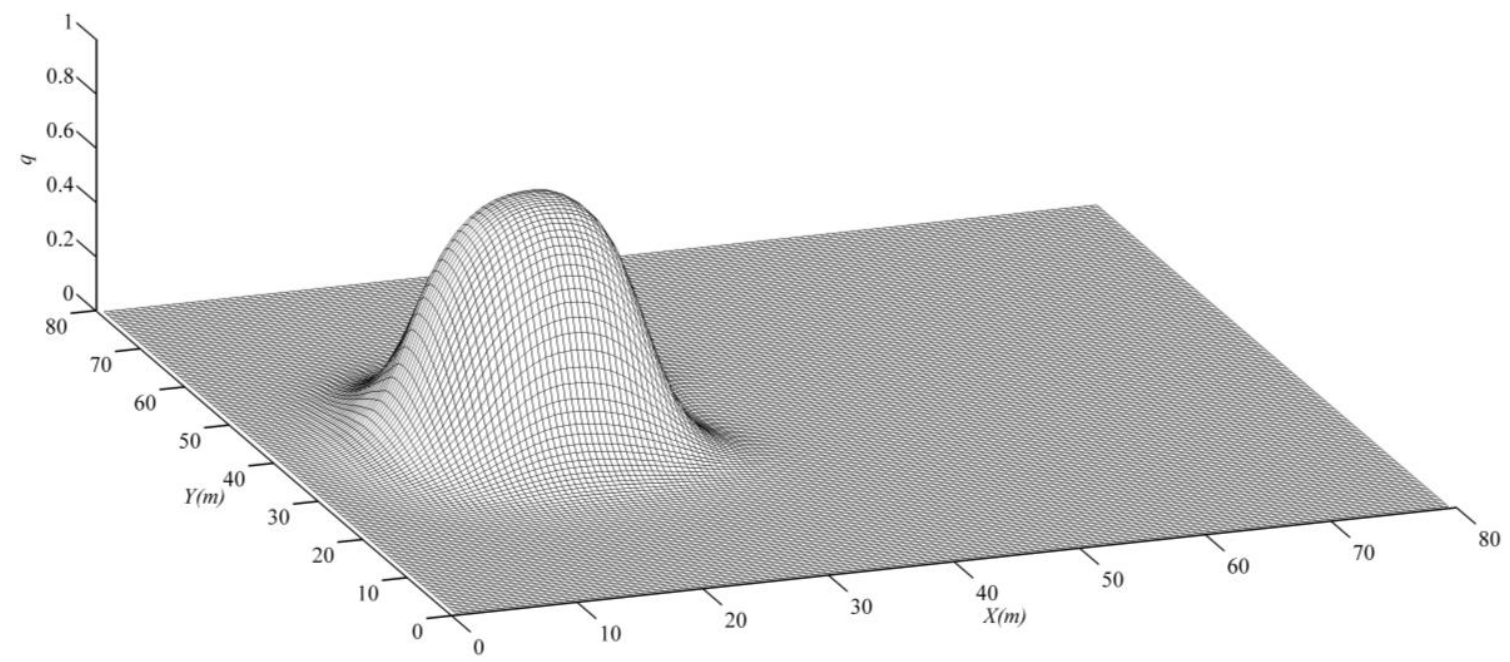

Figure 38. The resulting profile after one rotation for the Minmod limiter on the mesh consisting of $80 \times 80$ cells at a time step of 0.1 in Test 7 .

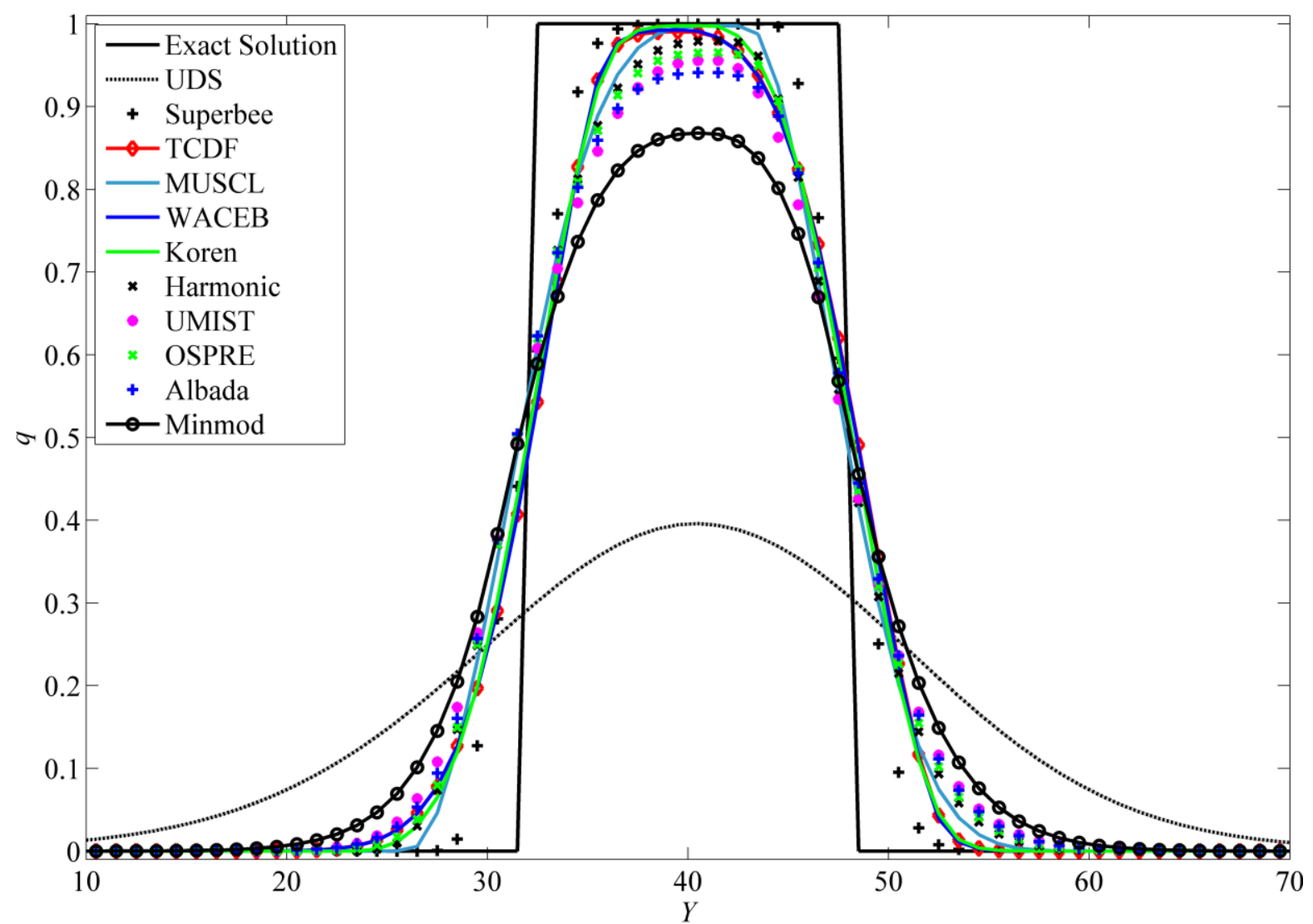

Figure 39. Concentration variations after one rotation along the line $x=20 \mathrm{~m}$ for the ten considered SS-TVD limiters in Test 7. 


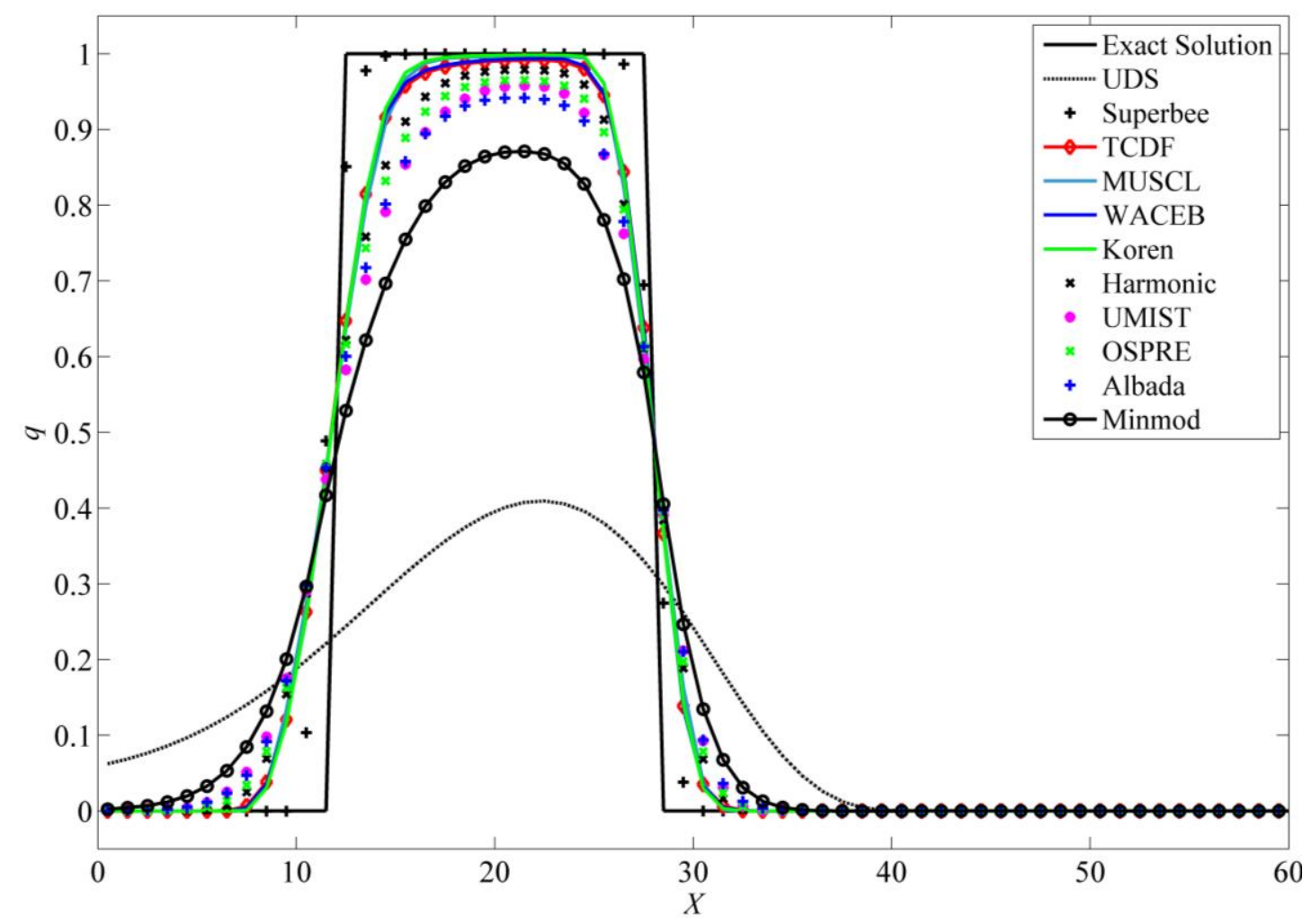

Figure 40. Concentration variations after one rotation along the line $y=40 \mathrm{~m}$ for the ten considered SS-TVD limiters in Test 7.

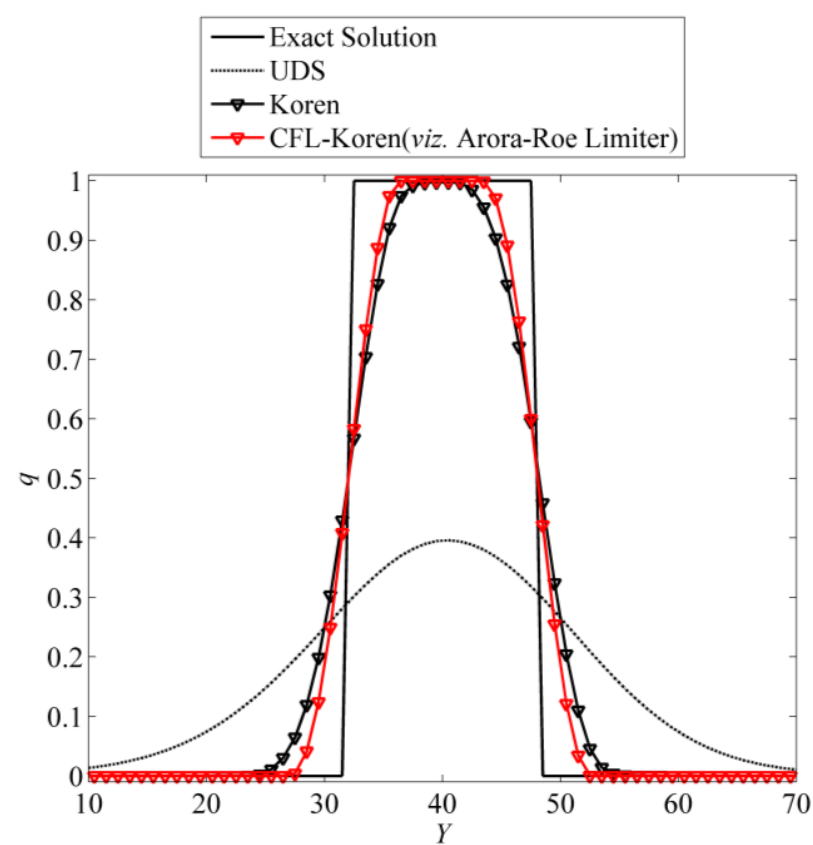

(a) Along the line $X=20$

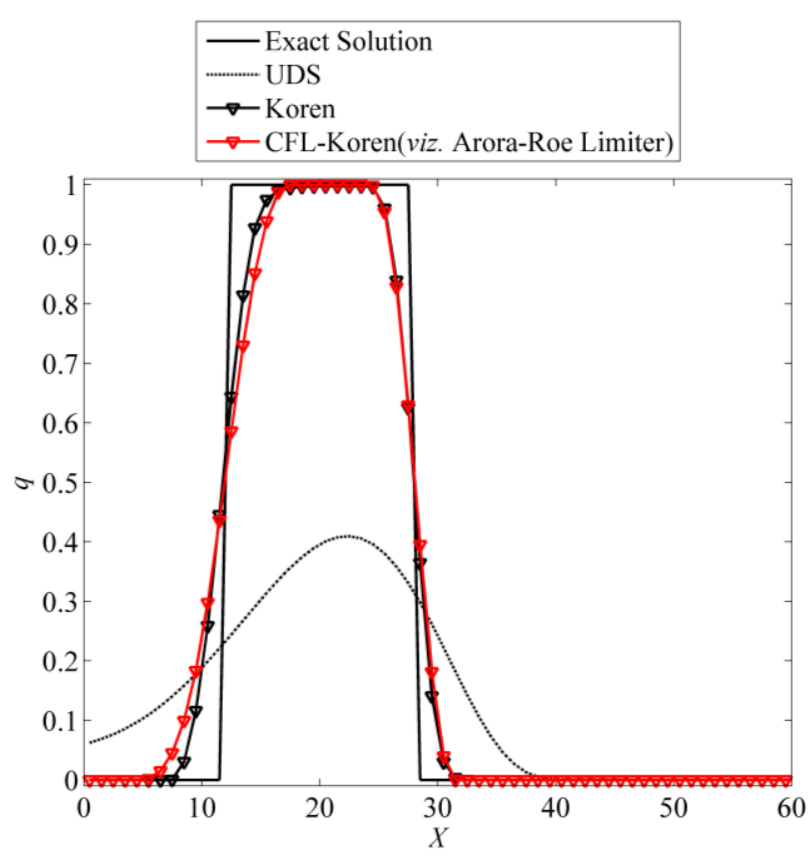

(b) Along the line $Y=40$

Figure 41. Concentration variations after one rotation along the lines $x=20 \mathrm{~m}$ and $y=40 \mathrm{~m}$ for the Koren and CFL-Koren (viz. Arora-Roe) limiters in Test 7. 


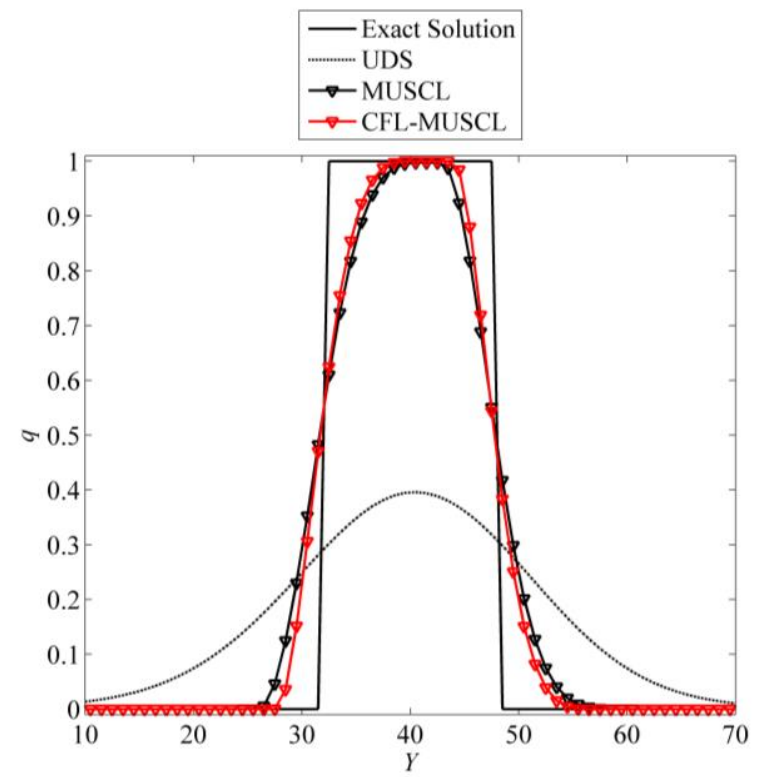

(a) Along the line $X=20$

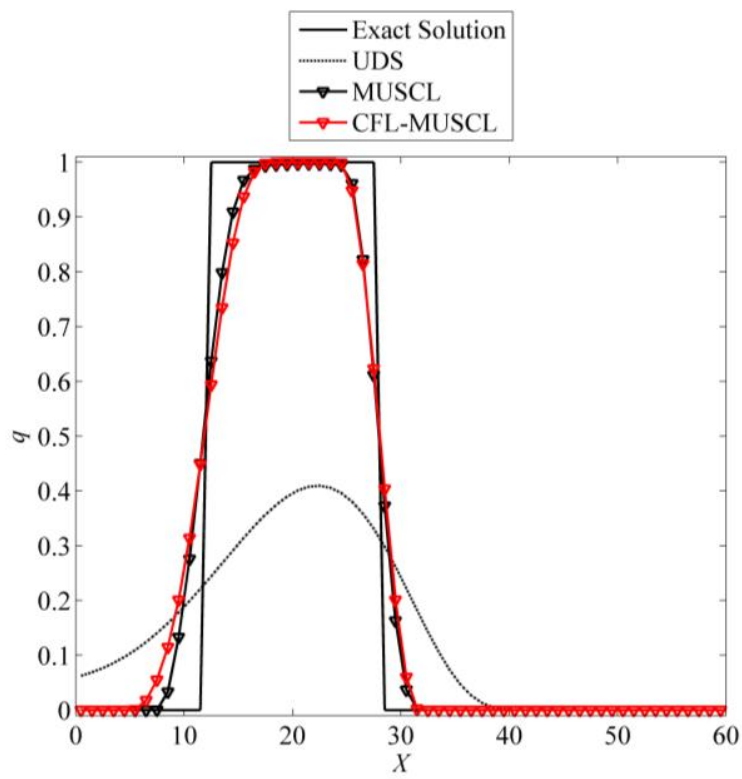

(b) Along the line $Y=40$

Figure 42. Concentration variations after one rotation along the lines $x=20 \mathrm{~m}$ and $y=40 \mathrm{~m}$ for the MUSCL and CFL-MUSCL limiters in Test 7.

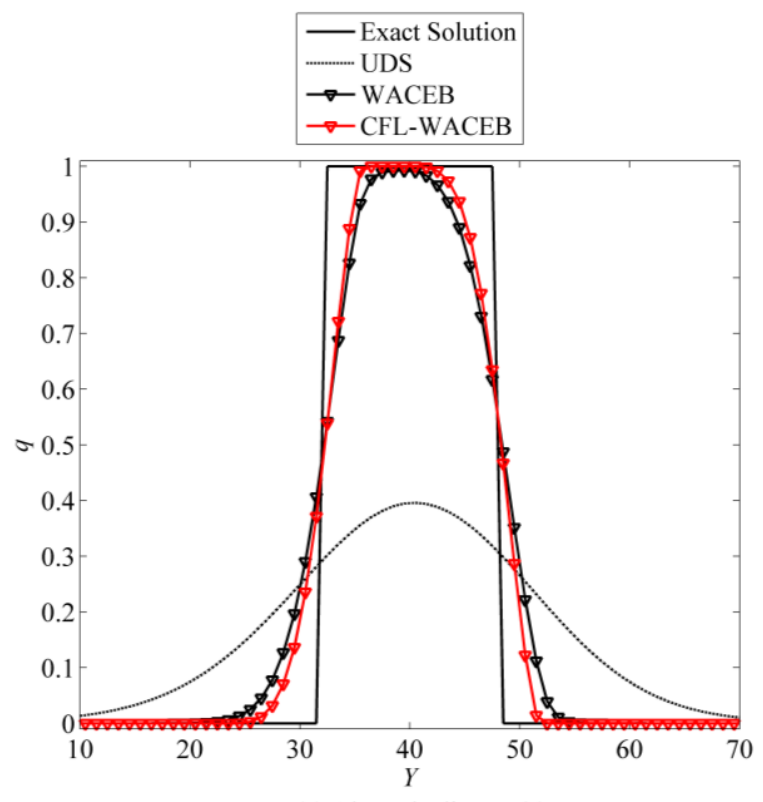

(a) Along the line $X=20$

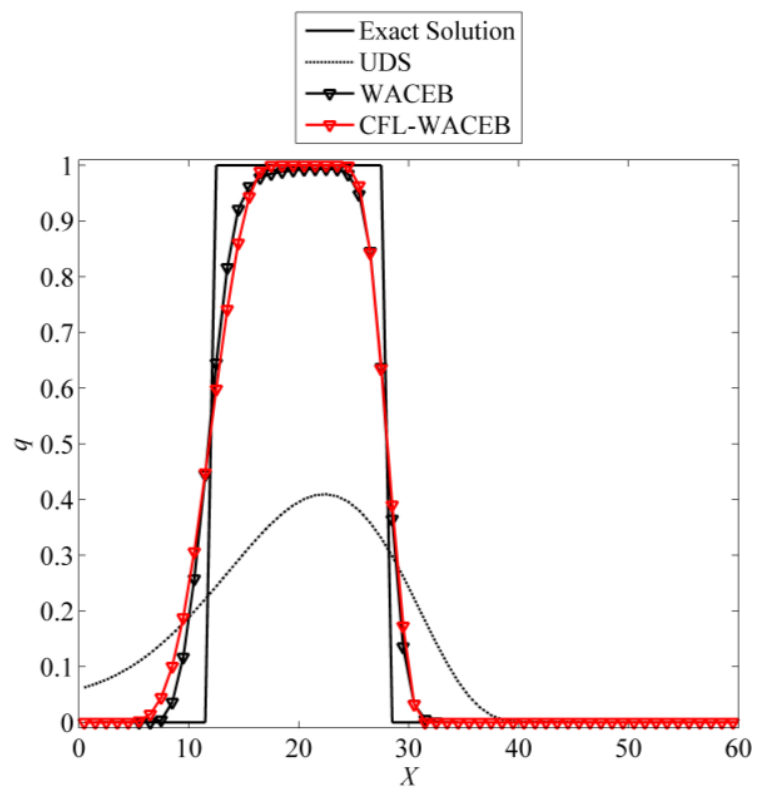

(b) Along the line $Y=40$

Figure 43. Concentration variations after one rotation along the lines $x=20 \mathrm{~m}$ and $y=40 \mathrm{~m}$ for the WACEB and CFL-WACEB limiters in Test 7. 


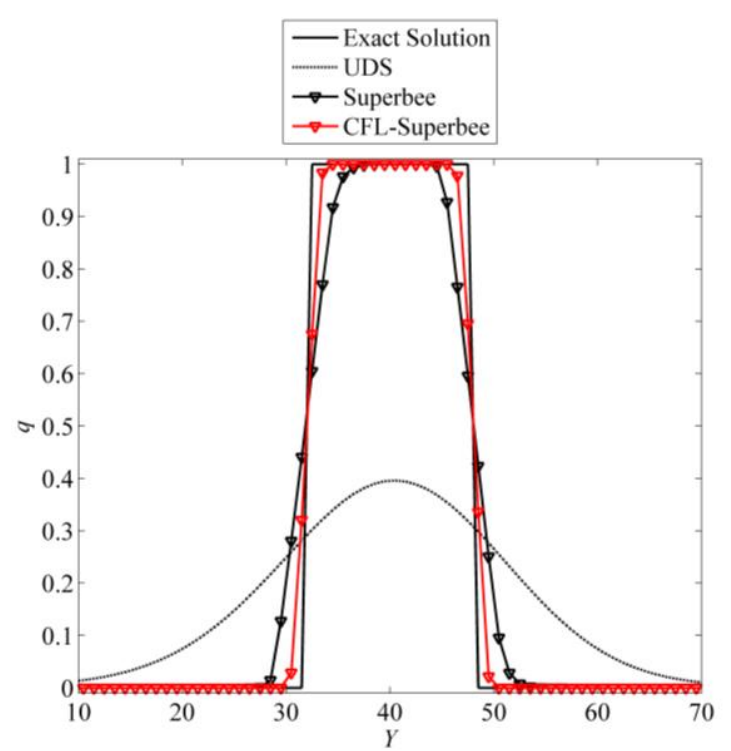

(a) Along the line $X=20$

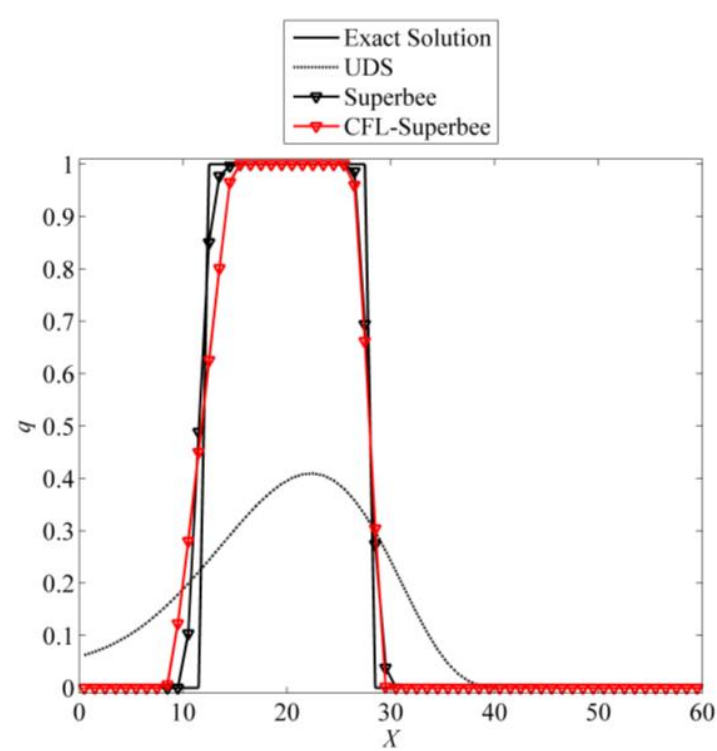

(b) Along the line $Y=40$

Figure 44. Concentration variations after one rotation along the lines $x=20 \mathrm{~m}$ and $y=40 \mathrm{~m}$ for the Superbee and CFL-Superbee limiters in Test 7.

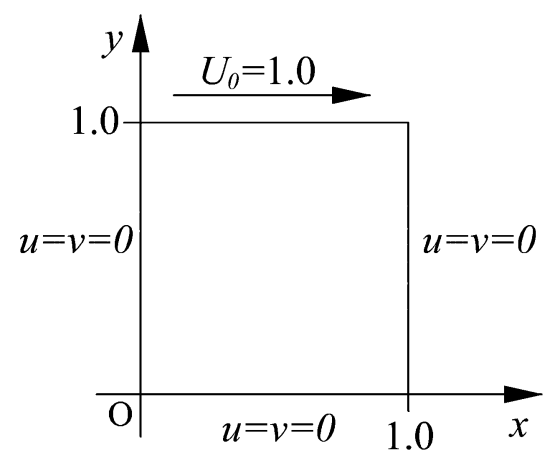

Figure 45. The computational domain and boundary conditions of the lid-driven flow.

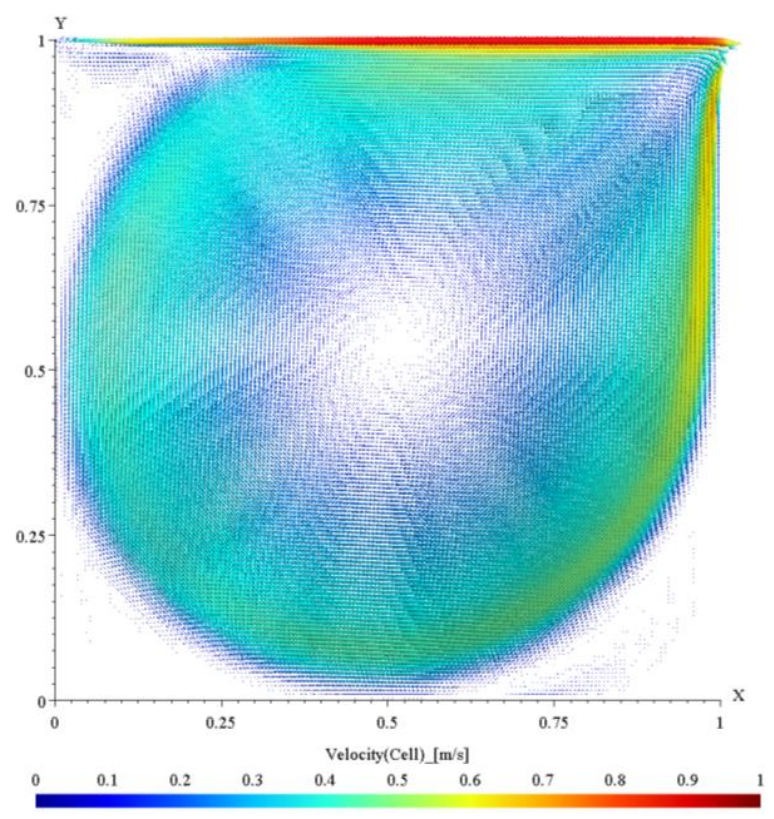

Figure 46. The steady-state velocity vector of the flow field for the TCDF limiter at a Reynolds number of 5000 on a mesh composed of $160 \times 160$ cells in Test 8 . 


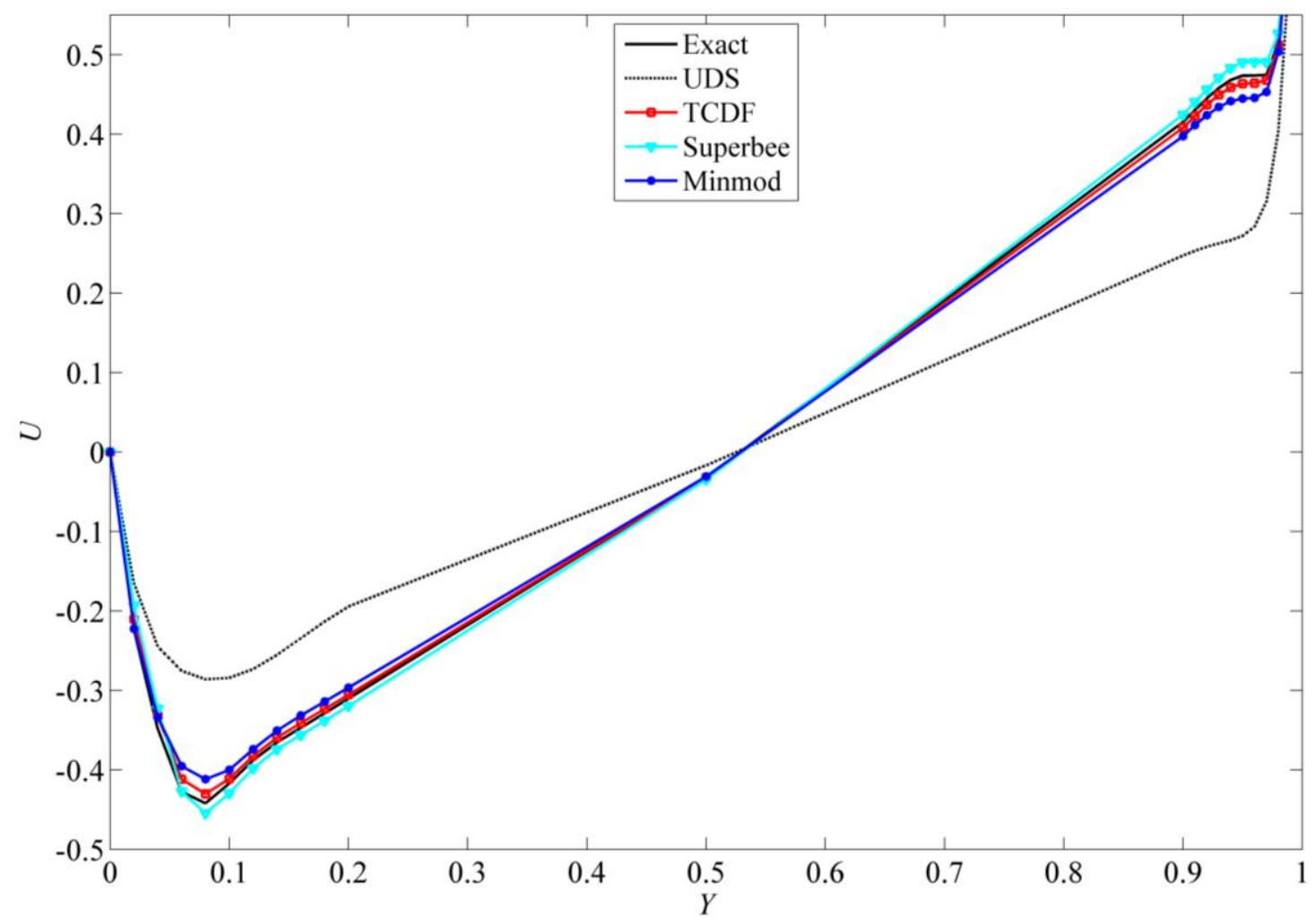

Figure 47. Comparison of the $U$-velocity profiles along the vertical centerline of the cavity for the TCDF, Superbee and Minmod limiter in Test 8.

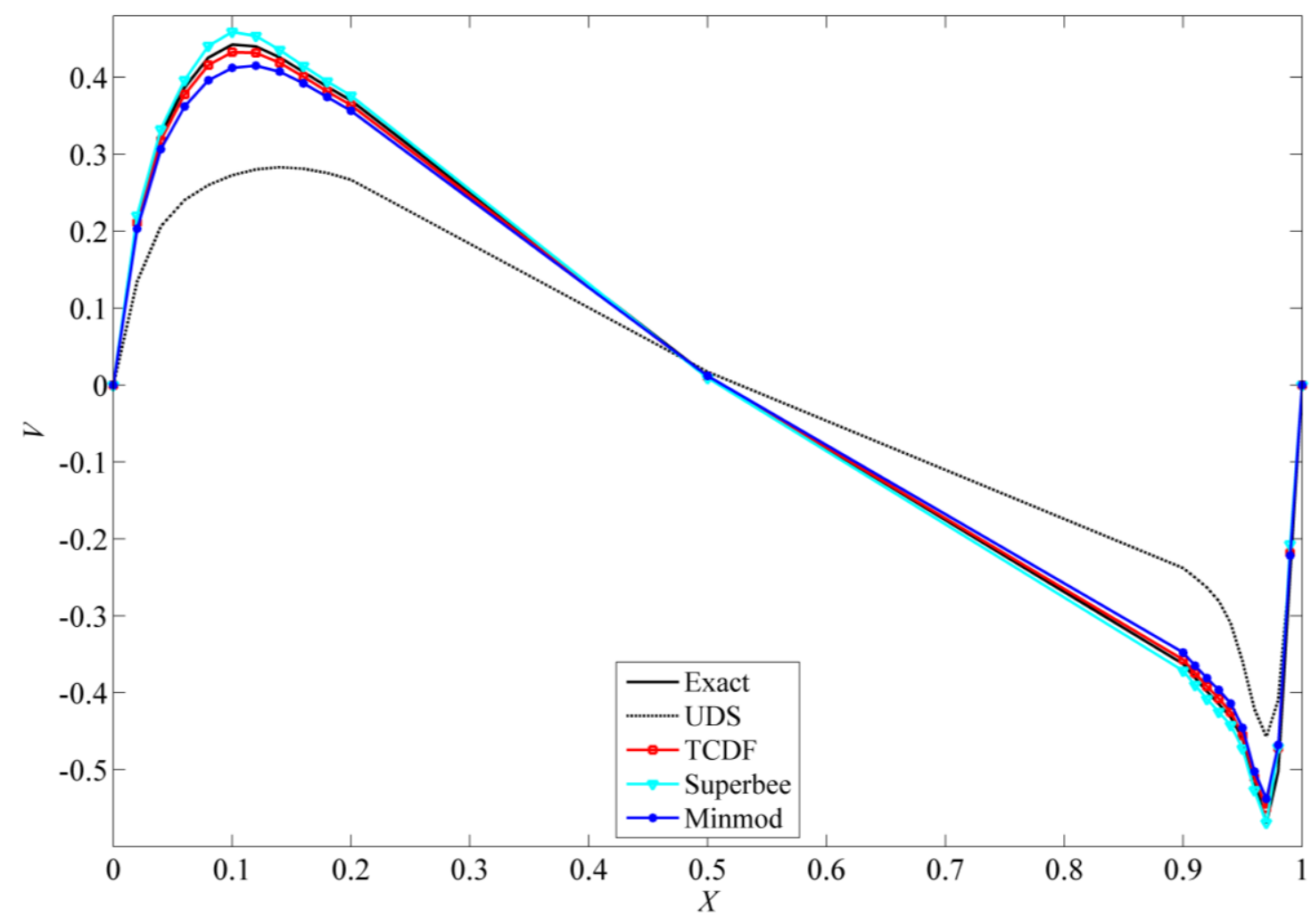

Figure 48. Comparison of the $V$-velocity profiles along the horizontal centerline of the cavity for the TCDF, Superbee and Minmod limiter in Test 8. 